\title{
THE CHOREOGRAPHY OF BELONGING: TOOTHED WHALE SPATIAL COHESION AND ACOUSTIC COMMUNICATION
}

By

\section{Nicholas Blair Wootton Macfarlane}

A.B. Princeton University, 2008

S.M., Massachusetts Institute of Technology, 2015

Submitted in partial fulfillment of the requirements for the degree of

\author{
Doctor of Philosophy \\ at the \\ MASSACHUSETTS INSTITUTE OF TECHNOLOGY \\ and the \\ WOODS HOLE OCEANOGRAPHIC INSTITUTION
}

February 2016

(C) 2016 Nicholas Macfarlane

All rights reserved.

The author hereby grants to MIT and WHOI permission to reproduce and to distribute publicly paper and electronic copies of this thesis document in whole or in part in any medium now known or hereafter created.

Signature of Author

Joint Program in Oceanography/Applied Ocean Science and Engineering Massachusetts Institute of Technology and Woods Hole Oceanographic Institution October 23, 2015

Certified by

Dr. Peter L. Tyack

Thesis Supervisor

Accepted by

Dr. Martin F. Polz

Chair, Joint Committee for Biological Oceanography Massachusetts Institute of Technology 


\title{
THE CHOREOGRAPHY OF BELONGING: TOOTHED WHALE SPATIAL COHESION AND ACOUSTIC COMMUNICATION
}

By

Nicholas Blair Wootton Macfarlane

Submitted to the MIT/WHOI Joint Program in Oceanography and Applied Ocean

Science and Engineering on October 23, 2015 in partial fulfillment of the requirements

for the degree of Doctor of Philosophy in the field of Biological Oceanography

\begin{abstract}
To maintain the benefits of group membership, social animals need mechanisms to stay together and reunite if separated. This thesis explores the acoustic signals that dolphins use to overcome this challenge and mediate their complex relationships in a dynamic $3 \mathrm{D}$ environment. Bottlenose dolphins are the most extensively studied toothed whale, but research on acoustic behavior has been limited by an inability to identify the vocalizing individual or measure inter-animal distances in the wild. This thesis resolves these problems by simultaneously deploying acoustic tags on closely-associated pairs of known animals. These first reported deployments of acoustic tags on dolphins allowed me to characterize temporal patterns of vocal behavior on an individual level, uncovering large variation in vocal rates and inter-call waiting time between animals. Looking more specifically at signature whistles, a type of call often linked to cohesion, I found that when one animal produced its own signature whistle, its partner was more likely to respond with its own whistle. To better evaluate potential cohesion functions for signature whistles, I then modeled the probability of an animal producing a signature whistle at different times during a temporary separation and reunion from its partner. These data suggest that dolphins use signature whistles to signal a motivation to reunite and to confirm identity prior to rejoining their partner. To examine how cohesion is maintained during separations that do not include whistles, I then investigated whether dolphins could keep track of their partners by passively listening to conspecific echolocation clicks. Using a multi-pronged approach, I demonstrated that the passive detection range of echolocation clicks overlaps with the typical separation ranges of Sarasota mother-calf pairs and that the amount of time since an animal was last able to detect a click from its partner helped explain its probability of producing a signature whistle. Finally, this thesis developed a portable stereo camera system to study cohesion in situations where tagging is not possible. Integrating a GPS receiver, an attitude sensor and 3D stereo photogrammetry, the system rapidly positions multiple animals, grounding behavioral observations in quantitative metrics and characterizing fine-scale changes that might otherwise be missed.
\end{abstract}

Thesis supervisor: Dr. Peter L. Tyack

Title: Senior Scientist Emeritus, Biology Department, Woods Hole Oceanographic Institution and Professor, Department of Biology, University of St Andrews 


\section{ACKNOWLEDGEMENTS}

Although I am the one whose name appears on the dissertation, it could not have happened without dozens and dozens of people. I was blessed with an incredible advisor in Peter Tyack who, despite moving to Scotland halfway through, was a never-ending font of creativity, inspiration and support. It has been an absolute delight and privilege to spend so much time together over the last five years. Then, when he did leave, Frants Jensen stepped up to fill in the gaps and became one of the best surrogate advisors I could have imagined.

Each of my committee members, Laela Sayigh, Andy Solow, Jonathan Howland, Michael Moore and Stephanie Seneff, was carefully chosen because their expertise covered a critical part of the thesis, and they have been here through thick and thin, helping me gather disparate and challenging data and bring them together into a cohesive story.

None of these data would exist without enormous field teams in Tarifa and Sarasota or the phenomenal amount of logistical support required for organizing and maintaining the expeditions. In Sarasota, it takes more than a hundred people on the water to tag a dolphin, and in Tarifa the fieldwork would have been impossible without the help of Philippe Verborgh, Pauline Gauffier and the rest of CIRCE. Then, once these data were collected, many students came through WHOI and helped with the herculean task of auditing and processing the acoustics.

Throughout the last five years, I have been supported and advised at every turn by the wonderful people working in the Academic Programs Office. Again and again they worked with me to transform the program in ways that dramatically improved my time here. It made an enormous difference to know that there were people in the administration with my interests at heart.

Finally, I am deeply grateful to my family and friends both in Woods Hole and in Boston. They took a lonely path and, out of nothing, forged a strong inspiring community. More than anything else, they are the reason I was able to accomplish this thesis.

This work was supported by the Office of Naval Research (N000140910528 and N000141210417), the WHOI Marine Mammal Center, WHOI Biology Department, WHOI Academic Programs Endowed Funds, the MIT Martin Family Foundation for Sustainability, the MIT Graduate Student Government, the Grossman Family Foundation, and the Danish Council for Independent Research (11-107628). 


\section{TABLE OF CONTENTS}

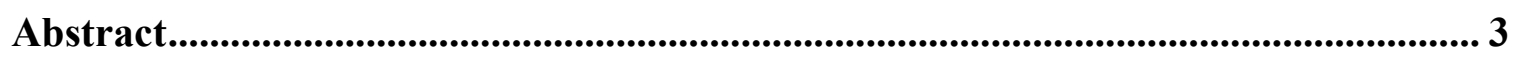

Acknowledgements ............................................................................................................... 5

Chapter 1: Introduction ....................................................................................................... 17

1.1 Introduction ...........................................................................................................................18

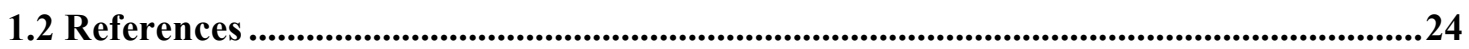

Chapter 2: Temporal Patterns in Bottlenose Dolphin Vocal Behavior ....................... 27

2.1 Abstract:...........................................................................................................................28

2.2 Introduction:.................................................................................................................28

2.3 Methods ..........................................................................................................................34

2.3.1 Field site

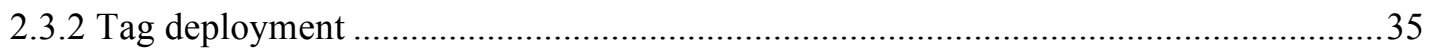

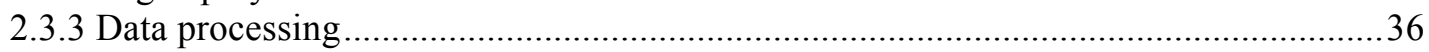

2.3.4 Cue timing analysis approach ..............................................................................

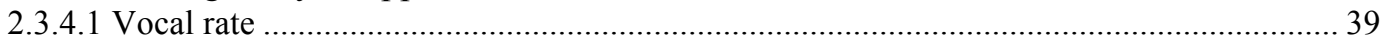

2.3.4.2 Waiting time and scaling ............................................................................................... 39

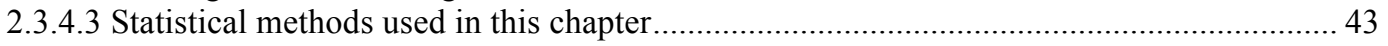

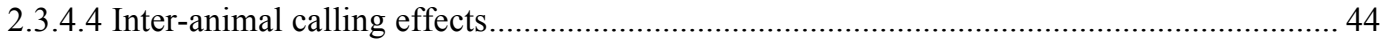

2.3.5 Individually-specific mark-recapture .....................................................................4

2.4 Results and discussion.........................................................................................................47

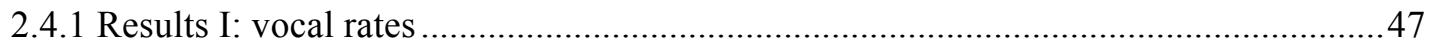

2.4.2 Discussion I: vocal rates ...............................................................................50

2.4.3 Results II: waiting time distributions.....................................................................51

2.4.4 Discussion II: waiting time distributions ................................................................56

2.4.5 Results III: response latency and inter-animal calling behavior...................................58

2.4.6 Discussion III: response latency and inter-animal calling behavior ..............................63

2.4.7 Results IV: individually-specific mark recaptures....................................................64

2.4.8 Discussion IV: individually-specific mark recaptures.................................................67

2.5 Conclusion .......................................................................................................................68

2.6 References ....................................................................................................................................71

Chapter 3: A reunion calling function for signature whistles in wild bottlenose

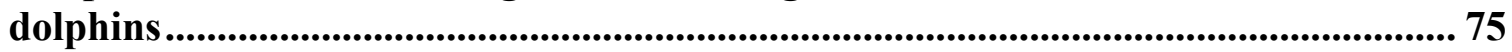

3.1 Abstract ..................................................................................................................

3.2 Introduction ...................................................................................................................76

3.2.1 Signature whistles as candidates for a contact call function.........................................78

3.2.3 Separating a contact call function from stress .........................................................78

3.2.4 Defining contact call functions for signature whistles..................................................

3.3 Methods ...............................................................................................................................81

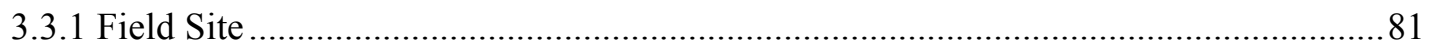

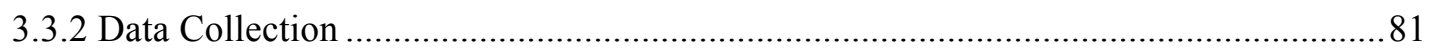

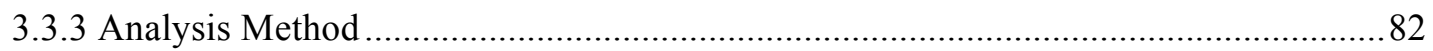

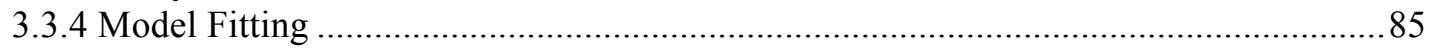

3.4 Results ……….......................................................................................................................8

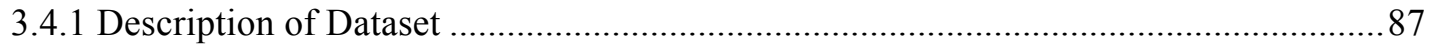

3.4.2 Data for Model Fitting:.................................................................................... 90

3.4.3 Variable Selection Process and Results ………………………………………......91 
3.5 Discussion ........................................................................................................................95

3.6 References: ..................................................................................................................101

Chapter 4: Do dolphins keep track of their partners by passively monitoring conspecific biosonar signals? ................................................................................................. 105

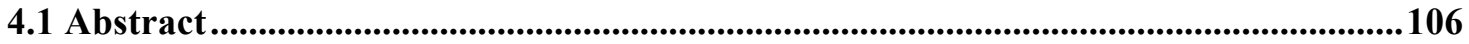

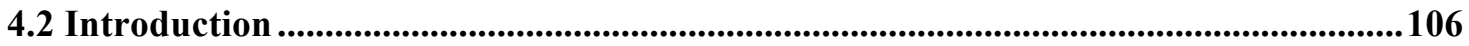

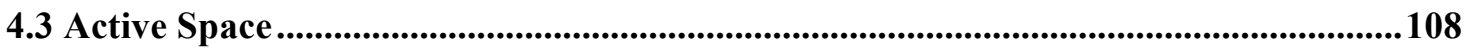

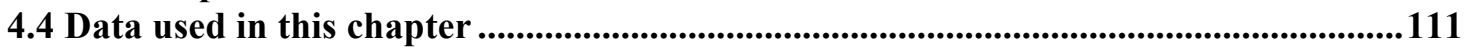

4.5 Comparison between active and passive detection ranges ........................................114

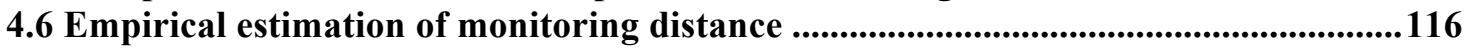

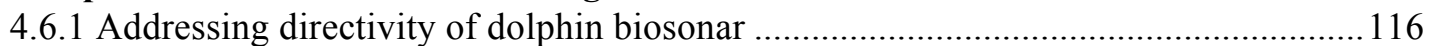

4.6.2 Empirical distribution of detection range for free-swimming dolphins .....................122

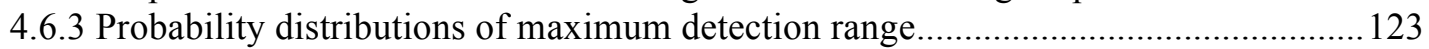

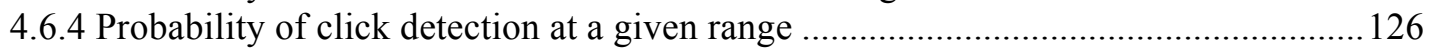

4.7 Effect of echolocation monitoring on the probability of emitting signature whistles 128

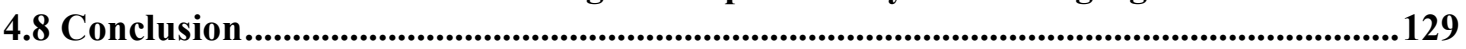

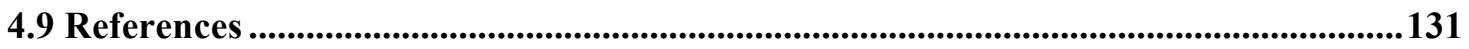

Chapter 5: A 3D stereo camera system for precisely positioning animals in space and time.................................................................................................................................. 133

5.1 Abstract ..............................................................................................................................137

5.2 Introduction ...........................................................................................................................137

5.3 System description ....................................................................................................................140

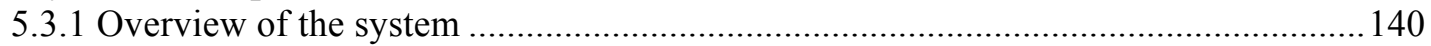

5.3.2 Technical approach to photogrammetric ranging ………………………………...... 142

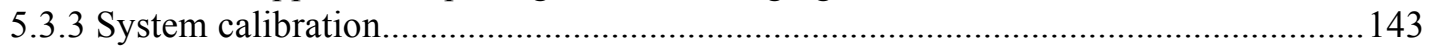

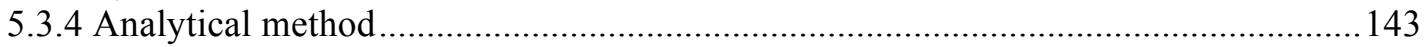

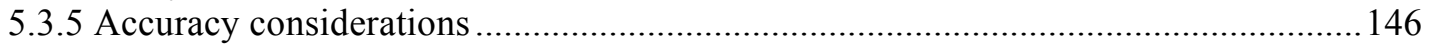

5.4 Field test and example data ....................................................................................152

5.5 Discussion ................................................................................................................................156

5.6 Acknowledgements............................................................................................................160

5.7 Ethical standards..............................................................................................................161

5.8 Conflict of interest ...................................................................................................................161

5.9 References ..............................................................................................................................162

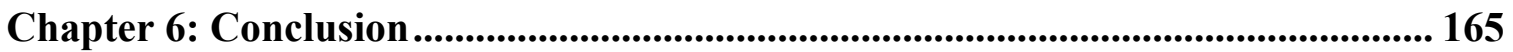

6.1 Framing remarks .................................................................................................................166

6.2 Chapter synopses................................................................................................................167

6.2.1 Chapter Two: Temporal patterns in bottlenose dolphin vocal behavior....................... 167

6.2.2 Chapter Three: A reunion calling function for signature whistles in wild bottlenose

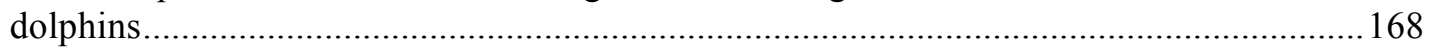

6.2.3 Chapter Four: Do dolphins keep track of their partners by passively monitoring

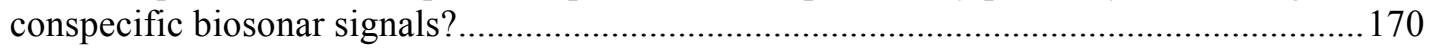

6.2.4 Chapter Five: A 3D stereo camera system for precisely positioning animals in space

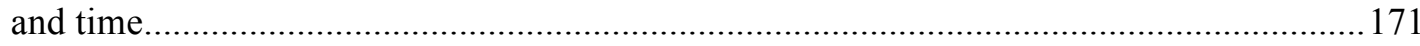

6.3 General observations .....................................................................................................171

6.3.1: Some observations on the rate-limiting steps of stereo photogrammetry and the potential for using drones to collect similar data .........................................................173

6.4 Future directions ....................................................................................................................175

6.5 References ...................................................................................................................................179 


\section{LIST OF FIGURES}

Figure 2.1: Examples of the 4 acoustic cue types analyzed in this chapter, signature whistles (upper left), non-signature whistles (upper right), chirps (lower left) and buzzes (lower right).

Figure 2.2: Illustration of waiting time (wt) method used in this chapter. Instances of signature whistles ( $\mathrm{sw}$ ) during separate deployments are shown on timelines for FB185 (A) and FB282 (C). B and D show histograms of waiting time between focal signature whistles for these deployments. Note that the axes have different scales. The cumulative density function of the raw waiting time distribution for one animal at a time is plotted to characterize its shape (E shows distribution for FB185 deployment from A and $\mathrm{B}$ on a $\log$ scale). To combine and compare distributions between deployments and cue types, each distribution is normalized by its mean waiting time $(\mathrm{F})$. The combined average scaled signature whistle curve is shown in green. Although this does not provide information on an absolute time scale, it allows comparison of the shape of individual and averaged waiting time distributions. The $\mathrm{x}$-axis units are mean waiting times where $10^{0}$ or 1 represents the mean waiting time for each distribution.

Figure 2.3: Scaled waiting time cumulative density functions across 12 tag deployments averaged by demographic class for signature whistles (sw; upper left), non-signature whistles (w; upper right), chirps (ch; lower left) and buzzes (bz; lower right). P-values for a 3-sample Kolmogorov-Smirnov test are shown on each plot. Significant differences were only found between distributions averaged by demographic group for non-signature whistles $(\mathrm{p}=$ $0.0181)$.

Figure 2.4: Within-animal pairwise comparisons of signature whistle and nonsignature whistle scaled waiting time distributions. Animals separated into mothers (upper left), calves (upper right) and males (lower left). Each line of the legend shows which curve was greater at the Kolmogorov-Smirnov statistic test point $(>/<)$ and the p-value is shown in parentheses for a 2sample test.

Figure 2.5: Within-animal pairwise comparisons of signature whistle and chirp scaled waiting time distributions. Animals separated into mothers (upper left), calves (upper right) and males (lower left). Each line of the legend shows which curve was greater at the Kolmogorov-Smirnov statistic test point $(>/<)$ and the $\mathrm{p}$-value is shown in parentheses for a 2 -sample test.

Figure 2.6: Within-animal pairwise comparisons of non-signature whistle $(\mathrm{w})$ and chirp (ch) scaled waiting time distributions. Animals separated into mothers (upper left), calves (upper right) and males (lower left). Each line of the legend shows which curve was greater at the Kolmogorov-Smirnov statistic test point $(>/<)$ and the $\mathrm{p}$-value is shown in parentheses for a 2 -sample test. 
Figure 2.7: Histogram showing the distributions of inter-signature whistle waiting times for all animals within single individuals (blue) and between one individual producing a signature whistle and its partner responding with their own signature whistle (red).....

Figure 2.8: Signature whistle response latency distribution within animal pairs (mother (M)-calf (C) on the left and male-male (ML) on the right). The pvalue for a 2-sample Kolmogorov-Smirnov test for that pair's distribution is shown in the upper left hand corner of each plot.

Figure 2.9: Probability of detecting an animal's signature whistle given a length of recording from that animal. Legend is organized by mothers $(\mathrm{M})$, calves (C) and males (ML).

Figure 2.10: Likelihood curves for estimating the number of individuals present, given the number of signature whistles (sw) detected over $8 \mathrm{~min}$. Each curve represents the spread of individuals that could produce a particular number of detected whistles. Note that the number of animals must be greater than or equal to the number of different detected signature whistles.

Figure 3.1: Log-survival plot of probability of inter-animal separation distance, pooled across $32 \mathrm{~h}$ of simultaneous tag deployments and 4 mother and calf pairs. A rapid change in slope occurs at $7 \mathrm{~m}$ of separation.

Figure 3.2: Example separation and reunion for a mother (tt14_129a, black) and her calf (tt14_129b, red). A. shows a 2D plan view of the animal tracks beginning at $(0,0)$. B. translates the track to a separation-distance timeseries between the dolphins. C. shows the absolute value of the heading angle in radians for each animal with respect to its partner with 0 radians representing the animal pointing directly at its partner. D. represents the speed with which an animal is closing the distance towards its partner. On all plots the time points representing a separation distance of halfway to maximum separation (teal), maximum separation (blue) and halfway to reunion (black) are marked as dotted lines. Signature whistles for each animal are shown as red and black circles.

Figure 3.3: Autocorrelation function of the residuals for the final model, demonstrating that any evidence for correlation is small. Dotted blue lines represent 5\% significance level.

Figure 3.4: Simultaneous deployments on 4 mother-calf pairs: FB33 and FB282 (A, 2.82h, 4 complete separations); FB197 and FB243 (B, 18.18h, 6 complete separations); FB133 and FB245(C, 6.18h, 3 complete separations); FB185 and FB249 (D, 4.75h, 11 complete separations) Separations are shown in dark red, reunions in blue and other times in green. Times with insufficient data to estimate distance are left blank. Signature whistles for the mother (magenta) and calf (black) are shown as circles placed at the beginning of each whistle. Notice that the x-axis for D is shifted to zoom in on the period when separations and reunions were observed. 
Figure 3.5. Signature whistle counts binned according to percent separation distance, suggesting a parametric quadratic regression as the basis for the model

Figure 3.6: Logistic regression model of the probability of a signature whistle during a separation-and-reunion event as a function of percent distance and whether or not the animal was in a separation or reunion. $95 \%$ confidence intervals are shown in red. Separations and reunions are shown separately. Note that the $\mathrm{x}$-axis for reunions begins at 100 percent separation, so that the two plots mimic the overall shape of an event

Figure 3.7: Excerpt from a tight bout of highly repeated signature whistles produced by FB243 when the animals were less than $7 \mathrm{~m}$ apart. FFT size 8192, Hanning window, temporal overlap of $90 \%$.

Figure 4.1: Left, reconstructed and corrected track for a separation between tt14_127a (mother, black) and tt14_127b (calf, red). Emitted clicks are green if detected, blue if missed. Upper right shows inter-animal separation distance vs time. Lower right shows absolute off-axis angle with respect to its partner with 0 being pointing directly towards it. Dotted lines connect 3 points at the same time across both tracks corresponding to $50 \%$ and $100 \%$ separation.

Figure 4.2: Back-calculated apparent source level ( $A S L)$ vs off-axis angle for outgoing clicks from 3 sets of partnered animals (AB, CD, EF). Mothers are shown on the left and calves on the right. Points are color-coded according to transmission loss $(T L)$. Clicks are aggregated across $3(\mathrm{AB}), 4(\mathrm{CD})$ and 3 (EF) separation and reunion tracks.

Figure 4.3: Polar plots of horizontal off-axis angle (in degrees) for each detected outgoing click emitted by the 3 sets of partnered animals shown in Figure 3 $(\mathrm{AB}, \mathrm{CD}, \mathrm{EF})$. Zero degrees would be perfectly on-axis. Apparent source level $(A S L)$ is shown in decreasing $10 \mathrm{~dB}$ concentric contours, with the outer contour representing the maximum recorded $A S L$. Clicks emitted while pointing towards the receiving animal $\left(-90^{\circ}\right.$ to $\left.+90^{\circ}\right)$ are colored blue. Clicks emitted while pointing away $\left(+90^{\circ}\right.$ to $\left.-90^{\circ}\right)$ are colored red.

Figure 4.4: Pairs of mother and calf dolphins are often at separation distances where they could use an echolocation monitoring strategy to track each other, but they also travel beyond that range. The maximum potential echolocation monitoring range is a function of the source level of the outgoing click, the transmission loss as it travels and the noise over which it must be detected at the receiver. The blue boxplot shows the distribution of maximum ranges at which each of the 13,082 emitted clicks would have been able to be detected above the noise on the receiver's tag at that point in time. Measured peak separations from mothers and calves are overlaid on the box plot as red dots. 
Figure 4.5: Apparent source level $(A S L)$ vs noise just prior to emitting a click for tt14_127a (top) and tt14_127b (bottom).

Figure 4.6: Probability density functions (PDF) of maximum detection ranges for clicks emitted by each dolphin. PDF estimated from distribution of apparent source levels $(A S L)$ and noise prior to emitted clicks as in Figure 4.5. Different functions are shown for clicks while pointing away from (red) and towards (blue) its partner. The smoothing for these functions used a Gaussian kernel with bandwidth chosen based on the number of clicks shown in parentheses.

Figure 4.7: Probability of an emitted click being detected at a given range while the clicking dolphin is moving away and moving towards its partner. Clicks are aggregated across all animals and separations. Probabilities were calculated in bins of increasing size. The number of detected (blue and red) and emitted clicks (black) in each bin are shown at the top of the plots.

Figure 5.1: Stereo camera geocoding system. a System in action collecting data on a group of long-finned pilot whales, Globicephala melas. b System in parts shown without wiring and battery.

Figure 5.2: Technical approach to photogrammetric ranging. A conceptual illustration of photogrammetric ranging using a frontal parallel $2 \mathrm{D}$ view. Disparity between the corresponding images of the target in the two calibrated cameras, shown here by the difference between $\chi L-\chi R$ allows triangulation of the range in space $(Z)$ according to the following equation: $Z=f T \chi L-\chi R$ where $f$ is the focal length of the two cameras and $T$ is the system baseline.

Figure 5.3: a Example stereo pair produced by the system from the left and right cameras. The corresponding positions of an analysis point chosen from simultaneous images in both cameras are shown for which the system can produce geodetic coordinates. b Left camera view of the stereo pair in a with the whales' geodetic coordinates. c Whales from b mapped onto a plan view. The system was located at $(1.9,-35.5)$. Several examples of inter-animal distance are also shown. Notice how difficult it would be to reproduce an accurate representation of position or distance by eye alone.

Figure 5.4: System accuracy at increasing range using a $50-\mathrm{cm}$ baseline and lenses with $25-\mathrm{mm}$ focal lengths. Five shore-based estimates were taken at 5$\mathrm{m}$ intervals. a System's estimated range compared to actual range. The dotted red line represents a hypothetical perfect estimate. b Absolute error for each estimate at the intervals shown in a. The solid line is a linear best fit through the error scores. Average deviation ranged from $0.1 \mathrm{~m}$ at 5 to $3.2 \mathrm{~m}$ at a $50-\mathrm{m}$ range. As with all stereo photogrammetric systems that rely on the disparity between two images in order to estimate distance, both accuracy and precision decrease with range 
Figure 5.5: System accuracy at increasing range measured on the water in a calm sea state (Beaufort 2). a Absolute error of a distance measurement between 2 traffic cones placed $3 \mathrm{~m}$ apart (a relevant inter-animal distance) on shore at increasing ranges with the system deployed from a small unstable vessel. Assessing accuracy of range with the system at sea is challenging, for even a small anchored vessel will move about, and the measurement tool used will have its own error curves. Range for comparison with the system was measured with a range finder, but should be understood to include a range of values of $+/-3$ to $4 \mathrm{~m}$ to account for boat movement. Distances were taken at opportunistic intervals when it was possible to stabilize the vessel as much as possible against moorings. The solid line is a linear best fit through the error scores. The average absolute value of the error ranged from $0.15 \mathrm{~m}$ at $\sim 12 \mathrm{~m}$ up to $1.69 \mathrm{~m}$ at $\sim 63 \mathrm{~m}$. To get a conservative estimate of geodetic location reconstruction accuracy, b shows absolute error of a geodetic coordinate calculated using the system compared with ones measured by placing the GPS receiver on top of the target. Like a, the positions were calculated at increasing distances from a target on shore. In addition to incorporating all measurement and location reconstruction error, this plot includes both error in the system GPS and again in the GPS measurement of the target's position without the system, leading to a conservative estimate. The solid line is a linear best fit through the error scores. Average absolute error ranged from $2.5 \mathrm{~m}$ at $\sim 9 \mathrm{~m}$ up to $25 \mathrm{~m}$ at $\sim 63 \mathrm{~m}$, which is beyond the design range of a system with this baseline and focal length.

Figure 5.6: a Interpolated tracks of animal positions of a group of six known longfinned pilot whales over $15 \mathrm{~min}$. Tracks are calculated from 336 measured positions across the 6 animals. Tracks are shown in Northing and Easting with respect to the initial position of the first animal sampled. Although the animals do not appear to have travelled far, they were swimming the entire time against a strong current. The circle, square, diamond and triangle markers represent identical points in time. b Dashed black line shows estimated inter-animal distance (in meters) between the mother and calf in Fig. a, calculated from 72 positions for the mother and 69 for the calf. Blue solid line shows surface cohesion of the entire group over time. Group dispersion was described as instantaneous group dispersion, defined as the RMS distance of individual positions relative to the group centroid at that instant in time. Note that this metric varies as a function of the number of individuals being tracked. Dispersion and distance were calculated using the 2D linear interpolated tracks from a 


\section{LIST OF TABLES}

Table 2.1: Tag deployments used for analysis........................................................... 47

Table 2.2: Focal cue counts and rates of signature whistles (sw), non-signature whistles (w), chirps (ch) and buzzes (bz) for analyzed tag data. The 6 simultaneously tagged pairs are shown in couplets shaded the same.

Table 2.3: Percent of inter-signature whistle-intervals described in this chapter filtered and matched according to Janik, et al. (2013).

Table 2.4: Signature whistles produced by known animals and the number and proportion of those whistles that were followed by the simultaneously-tagged partner producing its own signature whistle within 20s. Partner couplets are shaded the same color.

Table 2.5: Results for a rotation test on the null hypothesis that there is no effect of a partner producing a signature whistle on a dolphin's own signature whistle production in the following $5 \mathrm{~s}$. Each row shows the p-value for an animal's signature whistle production following its partner's signature whistle being greater than expected by chance.

Table 2.6: Continuous listening time in minutes required in order to detect an animal's signature whistle a given percent of the time.

Table 3.1: Summary of simultaneous tag deployments on 9 mother and calf pairs across the last 4 field seasons. Pairs included in the analysis are shaded gray. 88

Table 3.2: Counts of signature whistles produced by each tagged dolphin throughout the entire set of simultaneous tag deployments. Counts are separated both by animal, age class and phase. Close represents all times when animals are not in a separation-reunion event as defined in the methods. Mothers are shaded gray.

Table 3.3. Summary of the binomial logistic regression model showing that the probability of a signature whistle was best explained by percent distance and whether or not an animal was in a separation or a reunion

Table 4.1: Summary of simultaneous tag deployments on 3 mother-calf pairs whose reconstructed tracks are used in this chapter ......

Table 4.2. Summary of re-fitted binomial logistic regression model showing the significant improvement coming from adding the silence variable (shaded). 
Chapter 1: Introduction 


\subsection{INTRODUCTION}

Many animals live in groups that provide important benefits to individuals (Hamilton, 1971), such as decreased predation risk (Norris \& Dohl, 1980; Senigaglia et al., 2012), cooperative foraging (Connor et al., 2000), and a reduced cost of movement (Krause \& Ruxton, 2002). These benefits rely on animals being in the same place at the same time; therefore, to obtain the benefits of being a group member, an individual needs mechanisms for staying with a group or reuniting after separating (Da Cunha \& Byrne, 2009). These requirements form a particularly complex problem for marine mammals who live in dynamic three-dimensional environments with no shelter (Norris \& Schilt, 1988). This thesis is motivated by the question of how social bottlenose dolphins solve this essential problem and how it can be studied in the wild.

Bottlenose dolphins, Tursiops truncatus, are the most-studied odontocetes (Tyack, 2000; Janik, 2009). They live in complex fission-fusion societies where they form transient associations with multiple animals and stable relationships with certain individuals (Connor et al., 2000; Mann et al., 2000). Mediating these strong social bonds in an otherwise fluid society (Wells et al., 1987; Wells, 2003) requires a mechanism for staying in contact. Underwater, light attenuates quickly, making vision impractical over even short distances, and dolphins only have primitive chemical senses, so this mechanism will likely be acoustic (Janik, 2009).

Over the years, researchers have extensively studied bottlenose dolphin acoustic behavior (Herman \& Tavolga, 1980; Tyack, 2000). Dolphins produce a large and varied repertoire of whistles, pulsed sounds and echolocation clicks (Janik, 2009). They are highly skilled at imitating both natural and artificial sounds (Lilly, 1965; Richards et al., 
1984; King et al., 2013) and are one of the few species of mammals shown to be capable of vocal learning (Janik \& Slater, 1997). A primary focus of research has been signature whistles - stereotyped, learned, stable whistles that are specific to individuals (Caldwell \& Caldwell, 1965; Caldwell et al., 1990; Janik \& Sayigh, 2013). These whistles encode animal identity information (Caldwell et al., 1990; Sayigh et al., 1999), and have been associated with mediating cohesion in captive studies (Janik \& Slater, 1998) and to a lesser degree in the wild (Smolker et al., 1993; Watwood et al., 2005). The ease of keeping bottlenose dolphins in captivity has contributed to their use as a model system for studying acoustic communication. Captive facilities can offer a level of experimental control and opportunity for observations that are challenging to replicate in the wild. However, it is unclear how easily studies conducted in captivity generalize to the natural world. If dolphins evolved these behaviors for use in their natural habitat, they will ultimately need to be studied in the wild.

The fluid societies and three-dimensional environment that the animals encounter pose a problem for researchers as well as dolphins. Groups of social delphinids can number in the hundreds or thousands (Connor et al., 1998), and observations can usually only be conducted at the surface (but see Norris, 1974). Animals may only appear for fleeting instants, and it is not necessarily accurate to extrapolate surface behavior to the underwater world (Janik, 2009). In some species it can be extremely challenging to identify individual animals or even have a clear idea of group size, let alone a biologically relevant definition of group membership. Particularly problematic for studying acoustic communication, is the fact that it is often impossible to record all the sounds animals are producing, and only rarely can calls be attributed to individuals rather 
than groups. Researchers frequently do not even know which animals are in the area, let alone where they are in space relative to each other. These issues often select for $a d$ libitum observations that are chosen based on what is possible to observe rather than what is behaviorally significant.

In the most fundamental definition of communication, one actor produces a signal that transfers information to another one (Bradbury \& Vehrenkamp, 1998). Therefore, at a primary level, studying acoustic communication requires identifying both the caller and the respondent. Meeting these requirements in wild animals suggests the need to simplify the study system, focusing on small groups of individuals that share a strong bond. This thesis will concentrate on closely associated pairs of dolphins that are highly motivated to stay in touch — mother-calf pairs and male alliances. Mothers and calves associate very closely during the first couple years (Connor et al., 2000), and calves are vulnerable to shark attack when separated (Connor et al., 2000; Heithaus, 2001). Some calves even remain dependent on their mothers for up to 10 years (Wells, 1991), and maintain close proximity until the mother becomes pregnant again (Wells et al., 1987). Male alliances are groups of two or three highly-associated individuals that cooperatively herd females. They often swim side by side less than a body length apart, and their strong bonds have been reported to last up to 13 years (Connor et al., 2000).

Acoustic tags, such as the Digital Acoustic Recording Tag (DTAG, Johnson \& Tyack, 2003) help identify which animal produces a call and allow us to estimate the spatial positions of tagged animals. These tags are powerful tools for studying cetacean acoustic behavior, recording all the vocalizations of tagged and non-tagged animals, which can be linked with other tag data including depth, orientation and movement, 
resolving many of the problems raised by studying diving marine animals from the surface (Johnson et al., 2009). However, it has proved extremely challenging to tag small delphinids like bottlenose dolphins (Schneider et al., 1998; Macfarlane, 2015).

That situation has now changed, and since 2011, we have been able to simultaneously tag closely associated pairs of bottlenose dolphins during brief capturereleases as part of the Sarasota Dolphin Research Program health assessments (Scott et al., 1990). When I began my dissertation five years ago, we were testing initial deployments with non-functioning prototype tags, but we have continually fine-tuned our protocol and can now achieve long simultaneous deployments on partnered animals where it is possible to attribute caller identity with high confidence. This thesis uses these tags to study the acoustic and spatial behavior of wild bottlenose dolphins.

Chapter Two takes advantage of this unique opportunity to identify the vocalizing individual in order to investigate temporal patterns of acoustic behavior. By deploying on-animal tags on pairs of closely-affiliated animals, for the first time, I am able to characterize the vocal production of wild free-swimming dolphins on an individual level. Large inter-animal variation was found in vocal behavior, highlighting the importance of using on-animal tags that capture almost every vocalization rather than generalizing from a group's overall recordings. I found that different types of acoustic cues have different characteristic timing distributions, suggesting that they are associated with different functions. Moreover, looking closely at the distribution of signature whistles, I find that when one animal produces its signature whistle, it increases the probability that its partner will respond with their own signature whistle. Timing differences in the latency of this response may reflect asymmetries in the relationship. 
Chapter Three explores a contact calling function for signature whistles among wild dolphins. Signature whistles are a particularly well-studied call that have been generally associated with affiliation and social cohesion, making them likely candidates for signals that dolphins may use to maintain cohesion during separations. Using simultaneous tagging techniques to hone in on the signature whistle behavior of mothers and calves allowed me to model specific functions of signature whistles during separations and reunions, suggesting that they serve as reunion calls signaling a motivation to reunite, as well as identity advertisement calls just prior to reuniting after a temporary separation.

The use of signature whistles for mediating separations and reunions begs the question of how dolphins reunite during separations that do not include whistles. Like other toothed whales, dolphins gather information about their environment from echolocation, and these sonar clicks are audible to other animals. Chapter Four investigates whether dolphins could use these echolocation signals from conspecifics as a complementary mechanism to keep track of partners when separated. A multipronged approach demonstrates that despite individual needs to separate in order to forage, the effective range of this listening strategy overlaps with the typical separation ranges of Sarasota mother-calf pairs. Incorporating echolocation monitoring into the model from Chapter Three helped explain the probability of an animal producing a signature whistle, suggesting that passively listening to a conspecific's biosonar clicks may help a dolphin to keep track of a partner.

Chapters Two, Three and Four have described simultaneous tag approaches to studying cohesion and communication, but there are many more situations where it is not 
possible to tag all, or even any, animals in a group. How do we study cohesion when we cannot, or do not want to, tag animals but still need to know where they are? To address this question, Chapter Five developed a portable stereo camera system integrating a GPS receiver, an attitude sensor and three-dimensional stereo photogrammetry to estimate the position of multiple animals in space and time. In Chapter Five, I test the system, describe its function and use it to measure group cohesion on long-finned pilot whales in the Strait of Gibraltar. The system can provide quantitative grounding for behavioral observations that generally rely on error-prone and imprecise human distance estimation, allowing researchers to characterize fine-scale behavior or detect changes as a result of disturbance that might otherwise be challenging to observe. 


\subsection{REFERENCES}

Bradbury, J. W., \& Vehrenkamp, S. L. (1998). Principles of acoustic communication. Sunderland, MA: Sinauer Associates.

Caldwell, M. C., \& Caldwell, D. K. (1965). Individualized Whistle Contours in BottleNosed Dolphins (Tursiops Truncatus). Nature, 207(4995), 434-\&.

Caldwell, M. C., Caldwell, D. K., \& Tyack, P. L. (1990). Review of the signature-whistle hypothesis for the Atlantic bottlenose dolphin. In S. Leatherwood \& R. R. Reeves (Eds.), The Bottlenose Dolphin (pp. 199-234): New York: Academic Press.

Connor, R. C., Mann, J., Tyack, P. L., \& Whitehead, H. (1998). Social evolution in toothed whales. Trends in Ecology \& Evolution, 13(6), 228-232.

Connor, R. C., Wells, R. S., Mann, J., \& Read, A. J. (2000). The Bottlenose Dolphin. In J. Mann, R. C. Connor, P. L. Tyack \& H. Whitehead (Eds.), Cetacean Societies: Field studies of whales and dolphins (pp. 91-126). Chicago: Univ. Chicago Press.

Da Cunha, R. G. T., \& Byrne, R. W. (2009). The use of vocal communication in keeping the spatial cohesion of groups: Intentionality and specific functions. In P. A. Garber, A. Estrada, J. C. Bicca-Marques, E. W. Heymann \& K. B. Strier (Eds.), South American Primates (pp. 341-363): Springer New York.

Hamilton, W. D. (1971). Geometry for the selfish herd. Journal of Theoretical Biology, 31(2), 295-311.

Heithaus, M. R. (2001). Predator-prey and competitive interactions between sharks (order Selachii) and dolphins (suborder Odontoceti): a review. Journal of Zoology, 253, 53-68.

Herman, L. M., \& Tavolga, W. N. (1980). The communication systems of cetaceans. In L. M. Herman (Ed.), Cetacean behavior: Mechanisms and functions (pp. 149209). New York: John Wiley \& Sons, Inc.

Janik, V. M. (2009). Acoustic communication in delphinids. In M. Naguib, V. Janik, N. Clayton \& K. Zuberbuhler (Eds.), Advances in the Study of Behavior (Vol. 40, pp. 123-157): Academic Press.

Janik, V. M., \& Sayigh, L. S. (2013). Communication in bottlenose dolphins: 50 years of signature whistle research. Journal of comparative physiology A, 199(6), 479-489.

Janik, V. M., \& Slater, P. J. (1998). Context-specific use suggests that bottlenose dolphin signature whistles are cohesion calls. Animal Behaviour, 56, 829-838.

Janik, V. M., \& Slater, P. J. B. (1997). Vocal learning in mammals. In P. Slater, C. Snowdon, J. Rosenblatt \& M. Milinski (Eds.), Advances in the Study of Behavior (Vol. 26, pp. 59-99). San Diego: Elsevier Academic Press.

Johnson, M. P., Aguilar de Soto, N., \& Madsen, P. (2009). Studying the behaviour and sensory ecology of marine mammals using acoustic recording tags: a review. Marine Ecology Progress Series, 395, 55-73.

King, S. L., Sayigh, L. S., Wells, R. S., Fellner, W., \& Janik, V. M. (2013). Vocal copying of individually distinctive signature whistles in bottlenose dolphins. Proceedings of the Royal Society B: Biological Sciences, 280(1757).

Krause, J., \& Ruxton, G. D. (2002). Living in groups: Oxford University Press.

Lilly, J. C. (1965). Vocal mimicry in tursiops: Ability to match numbers and durations of human vocal bursts. Science, 147(3655), 300-301. 
Macfarlane, N. B. W. (2015). Tagging wild cetaceans: investigating the balance between more and less invasive techniques. (SM), Massachusetts Institute of Technology.

Mann, J., Connor, R. C., Tyack, P. L., \& Whitehead, H. (2000). Cetacean Societies. Field studies of dolphins and whales. Chicago: University of Chicago Press.

Norris, K., \& Dohl, T. (1980). The structure and functions of cetacean schools. In L. Herman (Ed.), Cetacean behavior: Mechanisms and functions (pp. 211-261). New York: John Wiley and Sons.

Norris, K. S. (1974). The Porpoise Watcher: A Naturalist's Experiences with Porpoises and Whales: New York: WW Norton.

Norris, K. S., \& Schilt, C. R. (1988). Cooperative societies in three-dimensional space: on the origins of aggregations, flocks, and schools, with special reference to dolphins and fish. Ethology and Sociobiology, 9(2), 149-179.

Richards, D. G., Wolz, J. P., \& Herman, L. M. (1984). Vocal mimicry of computergenerated sounds and vocal labeling of objects by a bottlenosed dolphin, Tursiops truncatus. Journal of Comparative Psychology, 98(1), 10.

Sayigh, L. S., Tyack, P. L., Wells, R. S., Solow, A. R., Scott, M. D., \& Irvine, A. B. (1999). Individual recognition in wild bottlenose dolphins: a field test using playback experiments. Animal Behaviour, 57(1), 41-50.

Schneider, K., Baird, R. W., Dawson, S., Visser, I., \& Childerhouse, S. (1998). Reactions of bottlenose dolphins to tagging attempts using a remotely-deployed suction-cup tag. Marine Mammal Science, 14(2), 316-324.

Scott, M., Wells, R., \& Irvine, A. B. (1990). A long-term study of bottlenose dolphins on the West coast of Florida. In S. Leatherwood \& R. R. Reeves (Eds.), The bottlenose dolphin (pp. 235-244). San Diego: Academic Press.

Senigaglia, V., de Stephanis, R., Verborgh, P., \& Lusseau, D. (2012). The role of synchronized swimming as affiliative and anti-predatory behavior in long-finned pilot whales. Behav Processes, 91(1), 8-14.

Smolker, R., Mann, J., \& Smuts, B. (1993). Use of signature whistles during separations and reunions by wild bottlenose dolphin mothers and infants. Behavioral Ecology and Sociobiology, 33(6), 393-402.

Tyack, P. L. (2000). Functional aspects of cetacean communication. In J. Mann, R. C. Connor, P. L. Tyack \& H. Whitehead (Eds.), Cetacean Societies: Field studies of whales and dolphins (pp. 270-307). Chicago: University of Chicago Press.

Watwood, S. L., Owen, E. C. G., Tyack, P. L., \& Wells, R. S. (2005). Signature whistle use by temporarily restrained and free-swimming bottlenose dolphins, Tursiops truncatus. Animal Behaviour, 69, 1373-1386.

Wells, R. S. (1991). The role of long-term study in understanding the social structure of a bottlenose dolphin community. In K. Pryor \& K. Norris (Eds.), Dolphin societies: discoveries and puzzles (pp. 199-225). Berkeley: University of California Press.

Wells, R. S. (2003). Dolphin social complexity: lessons from long-term study and lifehistory. In F. B. M. De Waal \& P. L. Tyack (Eds.), Animal social complexity: intelligence, culture, and individualized societies. Cambridge: Harvard University Press.

Wells, R. S., Scott, M., \& Irvine, A. (1987). The social structure of free-ranging bottlenose dolphins. Current mammalogy, 1, 247-305. 


\section{Chapter 2: Temporal Patterns in Bottlenose Dolphin Vocal Behavior}




\subsection{ABSTRACT:}

Bottlenose dolphins are the most widely studied toothed whale. However, research on communication has been severely limited by an inability to identify which animal has made a particular call. Simultaneously deploying acoustic recording tags on multiple animals can resolve this issue, but it has proved extremely challenging to tag small delphinids with suction-cup tags. Recently, that situation has changed, and in this chapter I report some of the general results and lessons from 42 successful tag deployments over the last four years as part of the Sarasota Dolphin Research Program. Large individual variability was found in vocal behavior, underlining the importance of attributing calls to particular animals. This chapter first analyzes individual call rates and then explores the inter-call-interval distribution to describe differences between the timing of different types of calls that are consistent with different usage and functions. Working with pairs of closely-affiliated animals, I found that one animal's signature whistle increases the probability of its partner producing its own signature whistle, and I describe asymmetries in response latency that may reflect differences in dominance or motivational state. Finally, passive acoustic monitoring is an increasingly common approach for estimating animal abundance. To consider the added value of knowing individual signature whistles for a mark-recapture estimate, this chapter investigates the inter-animal variability in the probability of detecting a signature whistle, tracking this uncertainty through simulated likelihood functions of the number of animals producing a given number of detected whistles.

\subsection{INTRODUCTION:}

The bottlenose dolphin, Tursiops truncatus, is the most widely studied odontocete, and over the years researchers have extensively explored its echolocation and acoustic communication behavior (Tyack, 2000; Janik, 2009). Across the decades of research on dolphin communication, studies have been hampered by a fundamental limitation in observational methods. Understanding communication requires identifying which individual makes which call, and how other individuals respond to each call. This is difficult to do with dolphins, especially in the field. Not only are individuals often out of sight underwater, but even when they are visible, they seldom make visible cues when 
producing a sound. In this chapter I describe efforts and results from the last four years of addressing this problem using on-animal acoustic tags.

Simultaneously deploying acoustic recording tags, such as the Digital Acoustic Recording Tag (DTAG), on multiple animals helps resolve issues with identifying which animal produces a call and with measuring vocal and non-vocal responses to calls in the wild. These tags are powerful tools for studying cetacean acoustic communication and behavior (Johnson \& Tyack, 2003; Johnson et al., 2009). They allow us to record vocalizations of tagged and non-tagged animals, which can be linked with data provided by tag sensors including depth, orientation and movement.

However, it has proved very difficult to tag small delphinids with suction-cup tags. In one of the few published studies, Schneider et al. (1998) deployed a suction-cup tag on bow-riding bottlenose dolphins. The dolphins reacted by increasing swimming speed, leaping and diving and avoiding the boat. I observed a similar reaction when tags were deployed on four short-beaked common dolphins (Delphinus delphis) in the Bay of Algeciras, Spain. The dolphins were bow-riding directly alongside the boat, and the tags were very gently deployed using a pole. Nevertheless, the tagged dolphins immediately twisted and turned, quickly accelerating and breaching out of the water until the tags were shed (Macfarlane, 2015).

Our understanding of dolphin acoustic communication and behavior has suffered from our inability to deploy on-animal acoustic tags. However, recently that situation has changed, and since 2011 we have been able to successfully tag bottlenose dolphins (Tursiops truncatus) as part of the Sarasota Dolphin Research Program (SDRP, see Scott et al., 1990). This change resulted from a new, smaller version of the DTAG, with half 
the volume, that was possible to deploy on smaller cetaceans such as bottlenose dolphins, achieving attachment times of up to $24 \mathrm{~h}$ when animals were tagged during the Sarasota Health Assessments described in 2.3.1. Although these tags are attached with suction cups, "computer tomographic scans of DTAG suction cups attached to the integument of a common dolphin cadaver showed consistent deformation of the skin, blubber and underlying muscle up into the dome of the cup" (Moore, MJ P. Comm, Oct 20, 2015). Therefore, these tags can potentially impact the animals. It is difficult to measure the extent to which the DTAGs affect the behavior of these wild dolphins, since our most accurate measures of their behavior come from the tags themselves. However, a captive study on male bottlenose dolphins found that the animals reduced their swimming speed when wearing tags, likely in order to compensate for the increased drag (van der Hoop et al., 2014). Thus, there may be some potential effects of the tag on the animal behavior; however, at present on-animal tagging is the only way to collect the data described in this thesis. In this chapter, I will describe some of the results and lessons from the first four years of successful deployments.

Bottlenose dolphins have a large and varied vocal repertoire (Tyack \& Clark, 2000). Much research has focused on individually-distinctive signature whistles that appear to be related to cohesion and are produced in high numbers when animals are isolated or stressed (Chapter Three). However, little is known about other categories of calls, such as non-signature whistles or chirps, and without being able to consistently identify the vocalizing animal in wild dolphins, even generalizing signature whistle vocal behavior from the lab to the field is challenging. To further explore the function of this 
varied repertoire, this chapter will ask if and how different categories of calls are used differently.

Inter-call timing between a sequence of the same type of vocalization emitted by the same animal is a powerful way to look for these usage differences. Animal signals are often modulated by repetition rate or interval, and repetition appears to be biologically important, with a lone signal potentially conveying a different meaning than a clustered or repeated one (Beeman, 1998; Bradbury \& Vehrenkamp, 1998). Timing can be affected by internal or external factors, such as behavioral state (Cook et al., 2004) or activity context (Herman \& Tavolga, 1980). For example meerkats (Manser, 2001), lemurs (Macedonia, 1990), and Colobus monkeys (Schel et al., 2010) all make highly repeated alarm calls when threatened. In other species, this inter-call waiting time can in turn convey information, with the rate and composition of mobbing calls in chickadees indicating the urgency of a threat (Templeton et al., 2005; Krams et al., 2012), and the delay between calls changing with escalation of aggression in howler monkeys (Kitchen, 2004). If different calls are used for different functions, they might be expected to have different characteristic inter-call-interval distributions. A note on terminology: in this chapter I will be analyzing these intervals using statistical methods for describing waiting time; therefore, I will refer to the distribution of intervals as the waiting time distribution.

Two possible contrasts in the waiting time distributions are differences between demographic groups or between vocalization types. Demographic classes often experience different social situations, or even the same situation in different ways, leading to differences in overall vocal rate and call usage in species ranging from Japanese macaques (Green, 1981) to Elk (Bowyer \& Kitchen, 1987). Likewise, different 
time courses have been reported for different vocalization types in a variety of species such as chickens (Evans et al., 1993) and squirrels (Sherman, 1977). In bottlenose dolphins, Janik et al. (2013) examined inter-call intervals and reported that when the majority of a bout contained repeated calls separated by $1-10$ s, the calls were most likely to be signature whistles. Calls separated by an inter-call-interval of less than one or more than ten seconds were less likely to be signature whistles, supporting the idea that waiting time distributions may reflect different call types and functions in this species.

Different kinds of functions would likely impact the waiting time distributions. Bradbury and Vehrenkamp (1998) suggest that calls triggered by an external event, such as alarm calls, would probably be clustered in tight bouts with long periods of silence between triggers. In contrast, general assembly calls, such as the clucking food calls of jungle fowl, would occur at low repetition rates not necessarily clustered as tightly (Bradbury \& Vehrenkamp, 1998). A similar pattern with no tight clustering might be observed for a type of contact call where the animals are simply keeping track of each other through spontaneous calling. However, a call whose function was to elicit a behavioral change in another animal, such as a motivation to reunite (Chapter Three), might demand a response, in which case more than one could be necessary if the partner was not responding. This could lead to a clustered distribution more akin to an alarm call. In this way, differences in timing are connected to differences in usage. This chapter will first explore whether these differences exist for different categories of bottlenose dolphin vocalizations or between different demographic classes.

Working with pairs of simultaneously tagged closely-affiliated animals, I will then look within a standard two-way model of communication where a sender produces a 
signal, and the recipient signals back (Bradbury \& Vehrenkamp, 1998). I will consider the case of vocal matching where both sender and receiver produce the same class of signal. Vocal-matching of this kind has been observed in many species such as killer whales (Miller et al., 2004) and bottlenose dolphins (Janik \& Slater, 1998; Quick \& Janik, 2012; King et al., 2014). Nakahara and Miyazaki (2011) reported that captive animals tended to use their own signature whistles during these sequences. If the signal is demanding a response, maybe one animal is attempting to elicit a behavioral change, and the partner needs to respond. Looking into signature whistle distributions of partnered animals, I will investigate whether the receiver is more likely to produce its own signature whistle within a certain time window after the sender emits a signature whistle, and if there are any asymmetries in vocal exchanges elicited within partners that might imply a hierarchical relationship or varying levels of motivation to remain cohesive.

Finally, the timing distributions can be used to help explore the potential for individually-specific mark recapture studies of bottlenose dolphins using passive acoustic monitoring. Increasingly, passive acoustic monitoring is emerging as an affordable way to assess marine mammal abundance (Mellinger et al., 2007; Thomas et al., 2012; Marques et al., 2013). This approach has several advantages over traditional visual surveys, such as the ability to sample in rough weather, at night, or over long periods of time. In particular, the individually-distinctive signature whistles of bottlenose dolphins may provide the opportunity for acoustic mark-recapture abundance estimates (Marques et al., 2013).

In the simplest form of Lincoln Peterson mark-recapture (for an overview see Marques et al., 2013), an initial sample of individually-identified animals is detected in 
an area; for example, by using passive acoustic monitors. Assuming random animal mixing, the proportion of animals in subsequent samples that are composed of the initially sampled ("marked") animals is used to estimate the proportion of marked animals relative to the entire population and by extension the population size. At its basic level, this method requires an individually-recognizable signal (signature whistles in this case) and a measure of the probability of detecting an animal given that it is in the area. The last requirement leads to a key assumption for mark-recapture: the probability of detecting an animal is assumed to be the same for all animals. If this assumption is not met, probability of detection will be biased towards easily detectable animals and population underestimated (Link, 2003; Marques et al., 2013). This assumption is stretched when animal home ranges are at different distances from the detector and when some animals' calling behavior gives them a higher probability of detection. Spatiallyexplicit capture-recapture methods that incorporate detector location into the estimates can mitigate the former (Borchers, 2012), but attempts to model the latter are challenging (Link, 2003). Here I will use our tag data to explore heterogeneity in the probability of detection stemming from the variability of signature whistle production across different bottlenose dolphins.

\subsection{METHODS}

\subsubsection{Field site}

Bottlenose dolphins develop strong social bonds between individuals within an otherwise fluid society (Wells et al., 1987; Wells, 2003). Studying communication therefore requires a long-term study of social relationships between identified individuals 
who can be followed longitudinally. Since 1970, the resident bottlenose dolphin community in Sarasota Bay, Florida has been the subject of the world's longest-running study of a dolphin population (Scott et al., 1990; Wells, 1991; Wells, 2003). Currently, about 165 dolphins reside in Sarasota Bay (Wells, 2009). Some of these individuals have been observed for more than 40 years, and the community includes individuals of up to 65 years of age. More than 270 residents have been temporarily captured for health assessments and life history studies and released back into the wild, in many cases repeatedly over decades (Wells et al., 2004). During these brief capture-release events, dolphins are encircled in shallow water with a $500 \mathrm{~m}$ x $4 \mathrm{~m}$ seine net. Individuals are then held in the water or brought aboard a specially designed veterinary examination vessel. Sounds from individual dolphins are recorded using hydrophones attached directly to the animal's head with a suction cup. These recordings provide a complete record of all whistles produced by each individual during the capture-release event. Thus, nearly every dolphin in the Sarasota community is linked to a rich set of life history, social association from long-term surveys, and communication data.

\subsubsection{Tag deployment}

Wild bottlenose dolphins were tagged during these brief capture-release events. Prior to release, a suction-cup DTAG-3 acoustic and kinematic tag (Johnson \& Tyack, 2003) was carefully placed by hand in front of each dolphin's dorsal fin while handlers held the animal in the water. This chapter will focus on simultaneous tagouts of closelyassociated mothers and calves or pairs of allied males. After release, the animals were radio-tracked from a small outboard-powered boat. During daylight hours, visual observations of group size, spread, synchrony, activity, nearest-neighbor distance and 
nearest-neighbor identity were collected at three to five-minute intervals in order to provide social context for the tag record. Upon release from the animal, tags were recovered by locating the VHF signal, data were downloaded for analysis, and the tags were prepared for another deployment.

\subsubsection{Data processing}

Acoustic and sensor data were processed and analyzed using custom programs written in MATLAB (Mathworks, Natick, MA). Call types were defined by observing spectrograms and listening to recordings in 10s increments. This chapter will focus on four types of signals, signature whistles, non-signature whistles, chirps, and echolocation buzzes (Figure 2.1). 

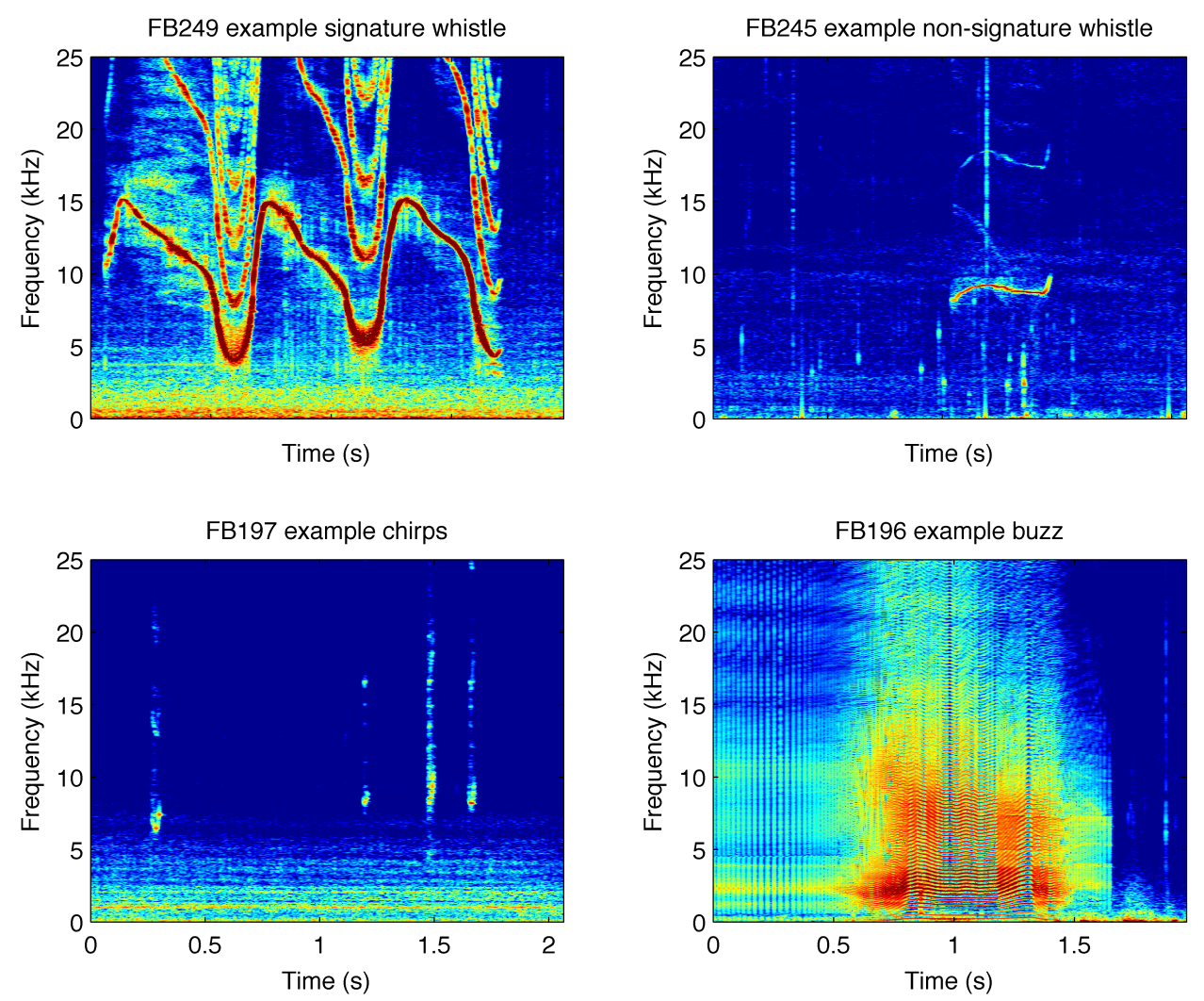

Figure 2.1: Examples of the 4 acoustic cue types analyzed in this chapter, signature whistles (upper left), non-signature whistles (upper right), chirps (lower left) and buzzes (lower right).

Since the dolphins had all been previously recorded during capture-release events, each dolphin's signature whistle (sw) was already known and could be easily identified in the tag record. Chirps (ch) were defined as short tonal vocalizations lasting less than $0.1 \mathrm{~s}$ in duration (Caldwell \& Caldwell, 1970; Caldwell et al., 1990). All other tonal vocalizations were classified as non-signature whistles (w). Buzzes (bz) were separated from a series of dolphin echolocation clicks at the point where echolocation clicks were produced so closely together that individual clicks were no longer discernable. On average this transition occurred at an inter-click-interval of $31 \mathrm{~ms}$. The sampling rate was always $500 \mathrm{kHz}$, and all spectrograms used an FFT size of 8192, Hanning window and 
temporal overlap of 0.9 . The sensitivity of the hydrophones in the tag was $-172 \mathrm{~dB}$ re $1 \mathrm{~V} / \mathrm{uPa}$. Calls were classified as focal or non-focal using a combination of signal amplitude, angle of arrival, low frequency energy and knowledge of the other animals in the area according to the method described in Johnson et al. (2015). In our conservative approach, calls were only designated as focal (produced by the tagged dolphin) if the classification could be made with a high degree of confidence using multiple lines of evidence.

\subsubsection{Cue timing analysis approach}

Three lines of investigation will explore the relative timing of different cues across different animal demographics, first the vocal rate, second the waiting time distribution within an animal and third the distribution of response latencies and their influence on vocal exchanges of signature whistles between an animal and its partner.

\subsubsection{Vocal rate}

Vocal rate for each cue type and animal was calculated as follows:

$$
\text { Rate }=\frac{\text { Number of cues of a type in a deployment }-1}{\text { Time between first and last cue of a type during a eployment }}
$$

Rate was only calculated between the first and last cue of a type in order to take into account the uncertainty of the time prior to an initial cue. Although this approach censors a final potential interval of unknown length that might be long, it would otherwise be impossible to be certain or consistent between tags; therefore, my conservative approach uses the first and last cues as bounds. All calculations in this chapter (and thesis) also exclude the first 30 min after release in order to help mitigate any potential after-effect of the health assessment. 


\subsubsection{Waiting time and scaling}

Beyond rate, in order to understand the relative timing and clustering of different vocalization types, we need a way to characterize the distribution of waiting times between cues. Figure 2.2 illustrates the method used. 

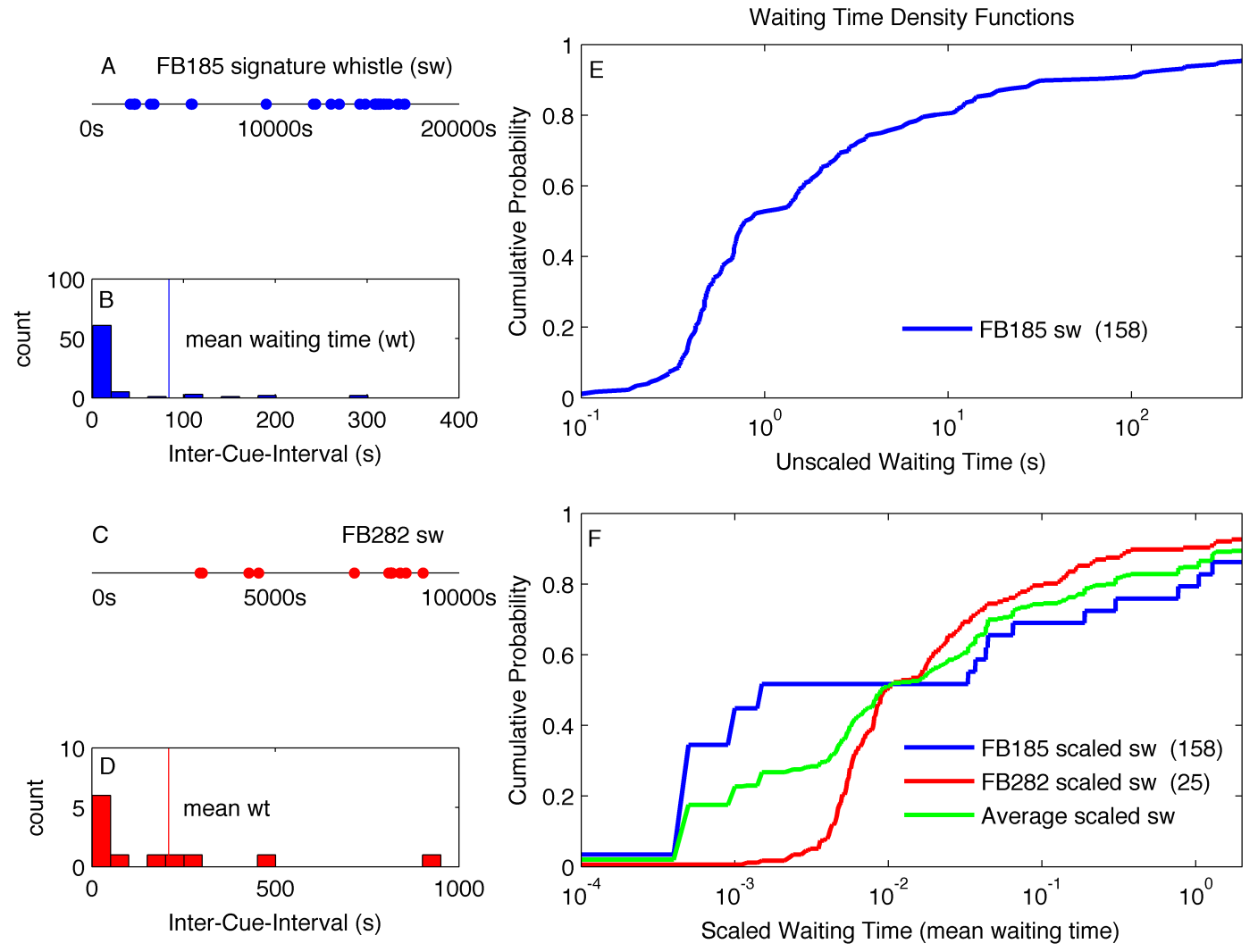

Figure 2.2: Illustration of waiting time (wt) method used in this chapter. Instances of signature whistles (sw) during separate deployments are shown on timelines for FB185 (A) and FB282 (C). B and D show histograms of waiting time between focal signature whistles for these deployments. Note that the axes have different scales. The cumulative density function of the raw waiting time distribution for one animal at a time is plotted to characterize its shape (E shows distribution for FB185 deployment from $\mathrm{A}$ and $\mathrm{B}$ on a log scale). To combine and compare distributions between deployments and cue types, each distribution is normalized by its mean waiting time (F). The combined average scaled signature whistle curve is shown in green. Although this does not provide information on an absolute time scale, it allows comparison of the shape of individual and averaged waiting time distributions. The $\mathrm{x}$-axis units are mean waiting times where $10^{\circ}$ or 1 represents the mean waiting time for each distribution.

Figure 2.2A and 2.2C show timelines of separate deployments for FB185 and FB282 respectively. Instances of focal signature whistles are shown as blue or red dots at the 
beginning of each cue. Figure 2.2B and 2.2D show the histograms of waiting time intervals corresponding to the focal signature whistle distributions of the two animals. As in Janik et al. (2013), inter-cue waiting times are calculated from the end of one cue until the beginning of the next cue of the same type. These waiting time distributions can then be represented as cumulative density functions in order to characterize the shape of each distribution (2.2E shows the distribution for the waiting times in $2.2 \mathrm{~B}$ on a log scale). In this case $80 \%$ of the waiting times are less than $10.62 \mathrm{~s}$. However, without normalizing, it is challenging to effectively compare this CDF to waiting time distributions from other animals or cue types, since each will be sensitive to its own characteristic rate.

To illustrate this problem, imagine the signature whistle distributions of two hypothetical animals, one with a very high baseline rate and one with a very low baseline rate. If a waiting time curve is plotted for each animal on the same time axis, the high rate animal will have a very steep curve, and the low rate animal will have a shallower one, irrespective of any clustering. The shape of the raw curve reflects each animal's baseline rate and obscures the relative shape of the distribution. To take a human language parallel, over the last year my waiting time distribution of the word 'dissertation' is probably a lot more tightly clustered than it was in my first year, suggesting that this word is associated with something that I do quite frequently at the moment. However, if I speak much slower now than I did then, this difference would be masked. In this chapter I am interested in whether two people use the same word differently, not whether one person speaks faster or more frequently than the other. Thus, in the raw form I can compare each distribution's rate, but to explore the relative clustering and usage, I need 
to normalize them, so that it is possible to average across animals or compare distribution shape to see which distribution is relatively more clustered.

To remove the sensitivity to base rate, I scale by normalizing each waiting time distribution by its mean waiting time. After removing the effect of rate, the curves become agnostic to scale, but we can now compare the shape of their waiting time distributions to assess relative clustering. Scaled curves for the signature whistle distributions shown in 2.2A-D are plotted in $2.2 \mathrm{~F}$ as well as an average of the scaled curves. The scaled CDF for FB185 (blue) rises much more steeply than for FB282 (red), indicating that a greater proportion of FB185's waiting times between signature whistles are relatively short and more clustered. To combine curves, the completed curves are averaged for each animal rather than pooling the waiting time distributions. Otherwise, if one of the two animals had far more waiting times than the other, it would have an outsize influence on the combined curve, even after normalizing for rate. By averaging the curves, each animal is weighted the same in the combined curve. Individual waiting time distributions (such as those represented by blue and red curves in F) can be compared using Kolmogorov-Smirnov statistics to test whether the CDFs come from the same distribution (David, 1958). To compare across groups of combined animals, Kolmogorov-Smirnov statistics can be used to compare differences in the averaged CDFs, for example the average curve of all mother signature whistles versus the average curve of all calf signature whistles.

To explore the relative shape of different types of cues, I look at the scaled waiting time distributions within individual animals in a series of pairwise comparisons - signature whistles vs non-signature whistles, signature whistles vs chirps, 
non-signature whistles vs chirps. The number of waiting times varied by individual. Individuals with five or less of a cue type are not included in the analysis for that cue type. The scaled waiting times for each cue type within an animal are compared using signed Kolmogorov-Smirnov statistics to test whether or not they come from the same distribution and the direction of the relationship.

\subsubsection{Statistical methods used in this chapter}

This chapter makes primary use of two different kinds of statistical tests: Kolmogorov-Smirnov tests (both two and three sample) and a rotation test. The twosample Kolmogorov-Smirnov test is a non-parametric test that determines if two datasets come from significantly different distributions without making any assumption about either of those distributions. These tests are commonly used with behavioral data, for example to compare model predictions of bat aggregations to measured data (Cvikel et al., 2015). The test calculates the empirical cumulative distribution function for each of the datasets and finds the maximum vertical deviation between the two curves. The significance of the test is determined by establishing the probability that a vertical difference greater than measured would have occurred by chance. The three-sample Kolmogorov-Smirnov test extends this comparison to three-samples (David, 1958).

The rotation test (Deruiter \& Solow, 2008) is a non-parametric method for testing whether the rate of a particular behavioral point process is affected by another factor at particular points or windows in time. For example, Deruiter \& Solow (2008) used a rotation test to assess sperm whale responses to air-gun sounds. In this chapter, the point process might be instances of signature whistles produced by one animal and the other factor could be the five-second time window after the partner animal has produced a 
signature whistle. The test would first find the number of signature whistles that occur in those windows with the actual data. Then it would join the beginning and end of one animal's signature whistle time series and randomly rotate it while keeping the windows in place before repeating the count. Repeating this process many times can be used to assess whether the number of signature whistles that fall during the windows is higher than expected by chance.

\subsubsection{Inter-animal calling effects}

I take two approaches to examining how the calling behavior of one animal affects its partner's calling. First, I test whether a dolphin is more likely to produce a signature whistle shortly after its partner produces one. Then, to explore asymmetries between partners in the relative timing of exchanged signature whistles, I consider how long an animal waits to produce its signature whistle when it is exchanging whistles with its partner (response latency).

To test how one animal's signature whistle production influenced the likelihood of its partner producing a signature whistle, I used a rotation test (Deruiter \& Solow, 2008) for the null hypothesis that one animal's signature whistle had no effect on its partner producing a signature whistle in the ensuing five seconds. I used a cutoff of five seconds to be consistent with previous work in primates and dolphins (Ghazanfar et al., 2002; Miller et al., 2009; Nakahara \& Miyazaki, 2011).

Going one step further in the intervals where signature whistles were exchanged within five seconds, I next considered differences in the distribution of each animal's response latency. This approach is similar to within-animal waiting time (hereafter referred to as 'waiting time'); however, instead of looking within a single animal, it relies 
on simultaneously tagged pairs and looks at the distribution from the start of one animal producing a cue until its partner produces a cue of the same type. Unlike waiting time, the response latency interval goes from start to start rather than end to start because the partner doesn't need to wait until the cue is finished to produce its own. If multiple cues are produced before the partner produces a cue of the same type, only the final interval is used. The distributions use absolute time without normalization, and as above, only responses at a latency of up to five seconds are considered. Individual pairings within partners are compared as above using a two-sample Kolmogorov-Smirnov test to investigate asymmetries in the response latencies between partners.

\subsubsection{Individually-specific mark-recapture}

I take two approaches to investigate the heterogeneity in the probability of detection stemming from the inter-animal variability of signature whistle calling rates. First, for each tag I estimate the relationship between the probability of detecting a signature whistle and the duration of the time window used to listen for that whistle. Then, I use these detection probabilities to simulate likelihood curves for the number of animals present given a number of different signature whistles detected in a fixed window.

The probability of detecting a signature whistle given a particular length of listening time was calculated by randomly sampling each tag record using bins of increasing size (from $60 \mathrm{~s}$ to $6 \mathrm{~h}$ ) and testing for the presence or absence of at least one focal signature whistle in the bin. The randomization was repeated 10,000 times for each bin size with the proportion of bins containing a signature whistle representing the probability of detection at that length of listening time. 
To select a listening window for the likelihood curve illustration, I first used the stereo camera system (Chapter Five) to estimate an average travel speed of $2 \mathrm{~ms}^{-1}$ from 75 dolphin GPS positions across three mother-calf and two male-male pairs. At that speed, it would take approximately eight minutes to travel the diameter of a circle whose radius is the $487 \mathrm{~m}$ estimated active space for bottlenose dolphin whistles in the habitat with the shortest range in Sarasota (Quintana-Rizzo et al., 2006). In reality this listening window would be affected by a variety of factors such as the direction of travel, bathymetry and layout of an acoustic array; however, it is a useful reference point to illustrate the potential effects of variability.

Likelihood curves for the number of individuals producing a given number of detected signature whistles were estimated as follows:

1. Calculate the probability of detecting a signature whistle for each individual in $8 \mathrm{~min}$ from the above curves.

2. Set the number $d$ of potentially detected different signature whistles

3. Set an initial number, $n$ of actual animals $(\geq d)$

4. Randomly choose $n$ detection probabilities from our distribution (step 1) with replacement and simulate the number of detections that would occur from them.

5. Repeat steps 3-4 10,000x to create a distribution of hypothetical detections.

6. The likelihood of $d$ whistles being produced by $n$ animals is the proportion of the distribution in step 5 that are equal to $d$.

7. Increment $n$ and repeat steps 4-6 to create the likelihood function

8. Repeat steps 3-7 for different values of $d$.

For this illustration I ignore any false positives and assume that all signature whistles produced are detected if they are produced within the listening window. 


\subsection{RESULTS AND DISCUSSION}

Over the last four years we have successfully deployed 42 DTAGs during Sarasota health assessments ( 7 of which were re-tags of the same animal) totaling $261 \mathrm{~h}$ of tagged time. The deployments focused on mother-calf and allied male pairs, tagging 24 male and 18 female animals, with ages ranging from 2 to 41 years old. Here I will consider in turn the results and discussion of vocal rates, waiting time distributions, response latency and the assessment of the viability of individually-specific mark recapture.

\subsubsection{Results I: vocal rates}

A subset of $115.01 \mathrm{~h}$ of tagout time across 12 animals was used for this Chapter (Table 2.1).

\begin{tabular}{c|c|r|r|r}
\multicolumn{1}{c|}{ Tag } & \multicolumn{1}{c|}{ ID } & \multicolumn{1}{c|}{ Sex } & \multicolumn{1}{c|}{ Age } & Tag Time (h) \\
\hline \hline tt13_130a & FB33 & F & 31 & 5.33 \\
\hline tt13_130b & FB282 & M & 5 & 2.91 \\
\hline tt14_125a & FB196 & M & 16 & 10.61 \\
\hline tt14_125b & FB268 & M & 21 & 8.86 \\
\hline tt14_126a & FB276 & M & 22 & 2.28 \\
\hline tt14_126b & FB142 & M & 22 & 9.62 \\
\hline tt14_127a & FB197 & F & 11 & 18.23 \\
\hline tt14_127b & FB243 & F & 2 & 18.18 \\
\hline tt14_128a & FB133 & F & 15 & 6.18 \\
\hline tt14_128b & FB245 & F & 2 & 7.04 \\
\hline tt14_129a & FB185 & F & 14 & 4.75 \\
\hline tt14_129b & FB249 & F & 3 & 21.02 \\
\hline & & & Total & 115.01
\end{tabular}

Table 2.1: Tag deployments used for analysis. 
Data were chosen based on the completeness of the deployments and confidence in the audits. These deployments were audited for acoustic cues and temporally processed as described in the methods. Table 2.2 shows counts and rates for the different cue types. 


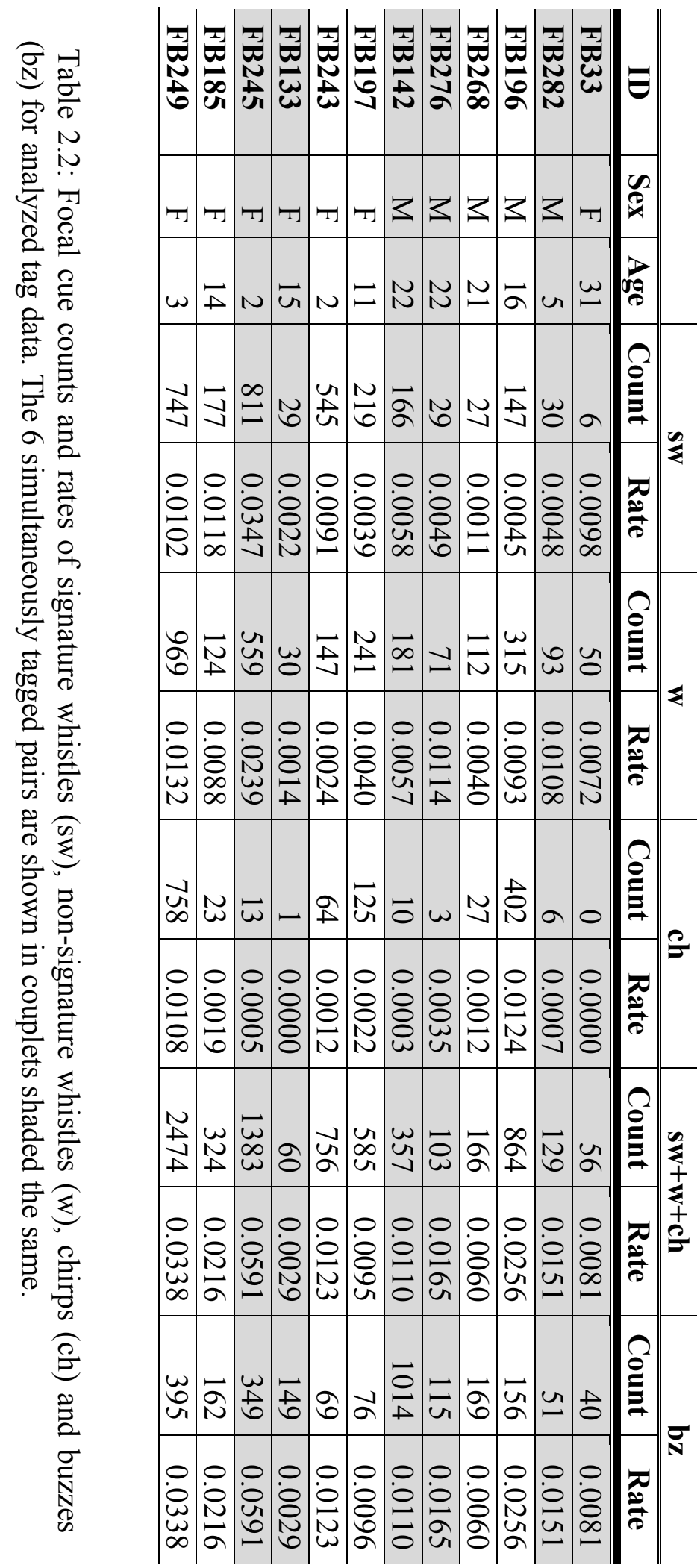


Table 2.2 shows that each animal has its own rate for a particular type of cue. There is a general trend towards calves as a group being more vocal overall than mothers (randomization test, $\mathrm{p}=0.1$ ) and males $(\mathrm{p}=0.086)$, but no difference between mothers and males $(\mathrm{p}=0.53)$. Comparisons within cue types did not show significant differences in rate, although again calves appear to have a trend towards a higher rate of signature whistles than mothers $(p=0.14)$ and males $(p=0.14)$. Signature whistles were the most common signals, but both chirps and non-signature whistles also occurred at high rates in these freely swimming dolphins.

\subsubsection{Discussion I: vocal rates}

In terms of general acoustic trends, one of the primary results is that there is a large amount of individual variability between animals, with acoustic behavior likely strongly influenced by context such as separation or presence of calves. This individual variability presents a particular problem for studies that are unable to attribute caller identity and it limits generalization from small sample sizes. I found no significant difference between the vocal rates of different demographic groups; however, there was a general trend towards calves being more vocal than both mothers and males. Like Cook et al. (2004), who towed hydrophones to record the whistle behavior of groups of bottlenose dolphins, I found that signature whistles comprised approximately half of the whistles produced by wild dolphins. However, Cook et al. (2004) reported lower whistle rates, with estimates of signature whistle frequency in a five-minute interval ranging from $0.34-2.52$ for a pair of dolphins depending on activity. In contrast, I found that signature whistle frequency in a similar interval ranged from $0.34-10.41$. Similarly, they estimated five-minute frequencies ranging from $0.23-3.83$ for non-signature whistles, 
and I found a range of $0.43-7.16$. There may have been some effect of group size; however, the rates I found were also higher than the Cook et al. (2004) estimates for larger groups. A more likely reason for this difference is that on-animal tags may allow us to record vocalizations that are not always detected by towed hydrophones.

This contrast highlights the utility of on-animal tags for reliably attributing caller identity and detecting signals that may be missed by other methods. In this way, they provide a more comprehensive characterization of a wild animal's vocal repertoire. Recent research in meerkats finds that low-amplitude 'close calls' may play a key role in social behavior (Townsend et al., 2010). In a similar vein, dolphin calls that may have been overlooked in the wild, such as chirps — which can be produced in large numbers (Table 2) - may be more important than previously thought. A key consideration is that, like much previous research, this chapter groups all non-signature whistles into a single category, but there are likely sub-categories within this broader group as well. At the moment too little is known about non-signature whistles to subdivide further.

\subsubsection{Results II: waiting time distributions}

To characterize the shape of the waiting time distributions of different cues, they were first averaged by cue type. Then signature whistles, non-signature whistles and buzzes were separated by demographic type. Figure 2.3 shows scaled waiting time cumulative density functions for signature whistles, non-signature whistles, chirps and buzzes averaged by demographic type. 

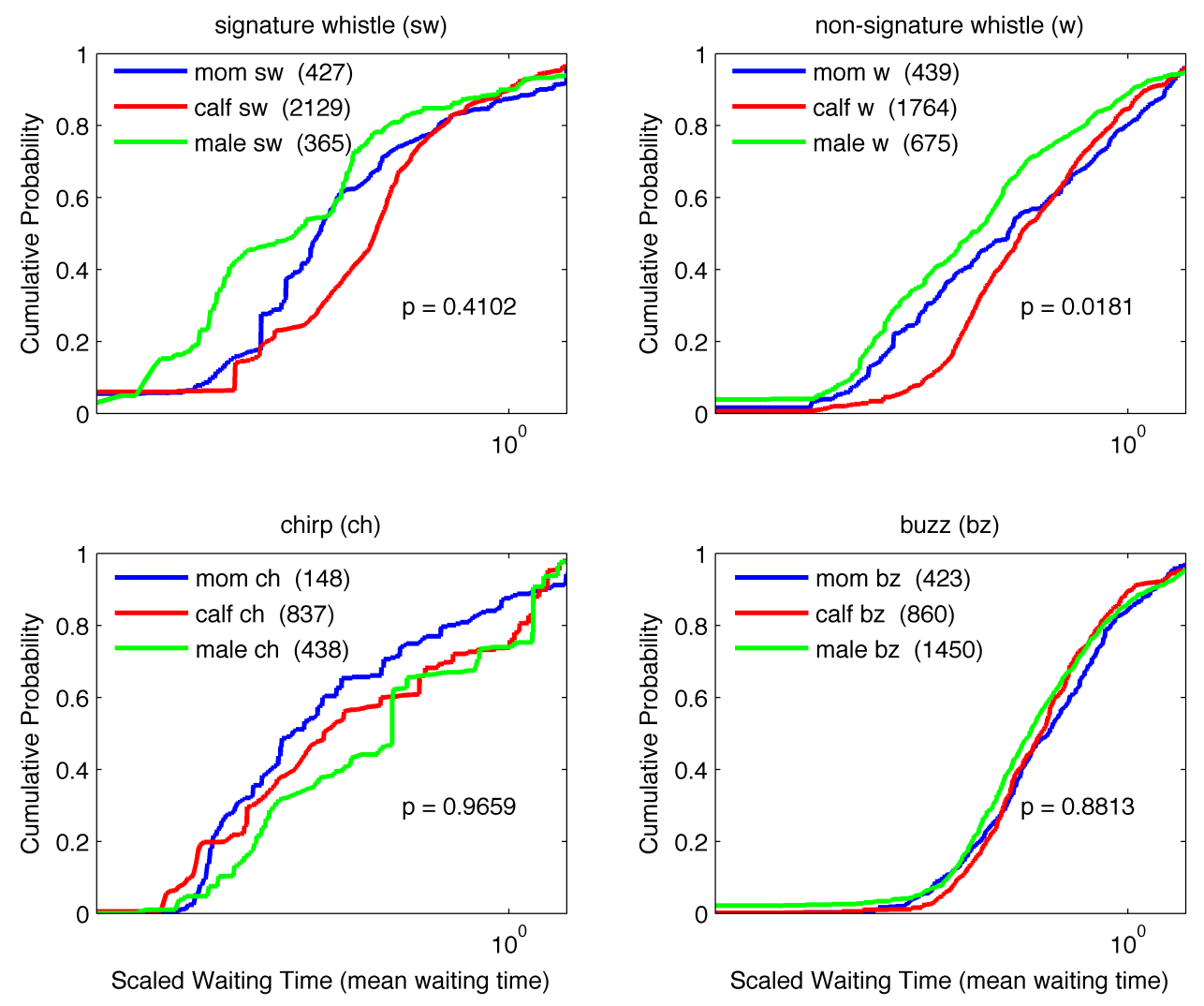

Figure 2.3: Scaled waiting time cumulative density functions across 12 tag deployments averaged by demographic class for signature whistles (sw; upper left), non-signature whistles (w; upper right), chirps (ch; lower left) and buzzes (bz; lower right). P-values for a 3-sample KolmogorovSmirnov test are shown on each plot. Significant differences were only found between distributions averaged by demographic group for nonsignature whistles $(\mathrm{p}=0.0181)$.

Of the four cuetypes, only non-signature whistles showed significant differences in the shape of their distributions across different demographics when compared using a Kolmogorov-Smirnov test $(\mathrm{p}=0.0181)$. The distributions of chirps and buzzes were very similar $(\mathrm{p}=0.9659$ and $\mathrm{p}=0.8813)$. 
There is large amount of variability between individuals; therefore, Figure 2.4 compares scaled distributions of signature and non-signature whistle within individual animals to explore patterns that might be obscured by averaging across demographic groups.
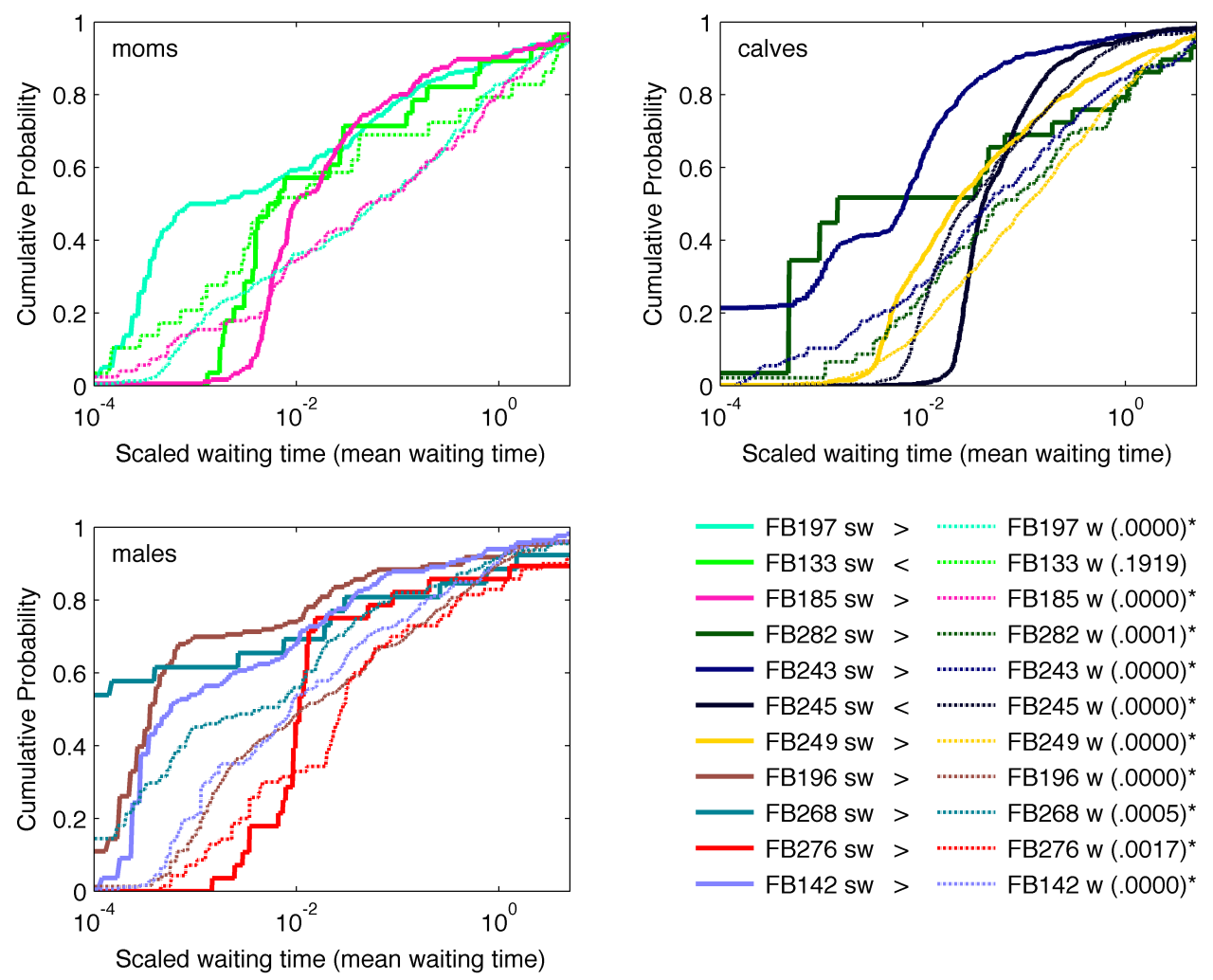

Figure 2.4: Within-animal pairwise comparisons of signature whistle and non-signature whistle scaled waiting time distributions. Animals separated into mothers (upper left), calves (upper right) and males (lower left). Each line of the legend shows which curve was greater at the KolmogorovSmirnov statistic test point $(>/<)$ and the p-value is shown in parentheses for a 2-sample test.

Of the eleven animals with sufficient waiting times for comparison, ten showed significantly different shapes between signature and non-signature whistles when tested with a 2-sample Kolmogorov-Smirnov test. Of those that were significantly different, the 
signature whistle curve was greater than the non-signature whistle curve at the

Kolmogorov-Smirnov statistic test point in nine out of ten animals. This implies that within almost all animals, signature whistles are relatively more clustered than nonsignature whistles.

Figure 2.5 takes the same approach and applies it to the comparison between signature whistles and chirps.
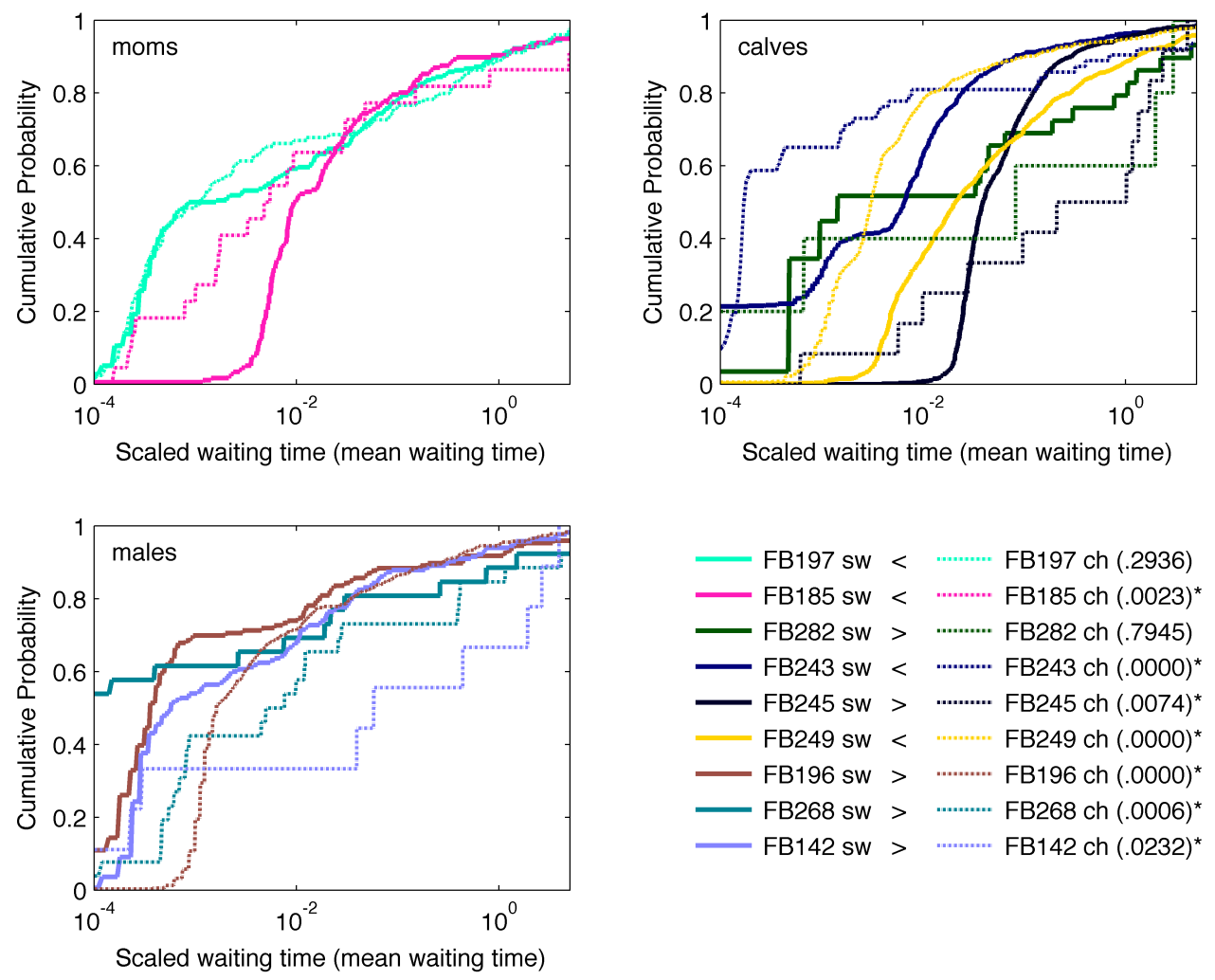

Figure 2.5: Within-animal pairwise comparisons of signature whistle and chirp scaled waiting time distributions. Animals separated into mothers (upper left), calves (upper right) and males (lower left). Each line of the legend shows which curve was greater at the Kolmogorov-Smirnov statistic test point $(>/<)$ and the p-value is shown in parentheses for a 2sample test. 
Of the nine animals with sufficient waiting times for comparison, seven showed significantly different shapes between signature whistles and chirps when tested with a 2sample Kolmogorov-Smirnov test. The relative direction of the shape was less consistent than for signature whistles and non-signature whistles. Of those that were significantly different, in four cases the distribution of signature whistles was relatively tighter than chirps, and in the remaining three cases, the opposite was true.

Figure 2.6 compares non-signature whistles and chirps in the same way.
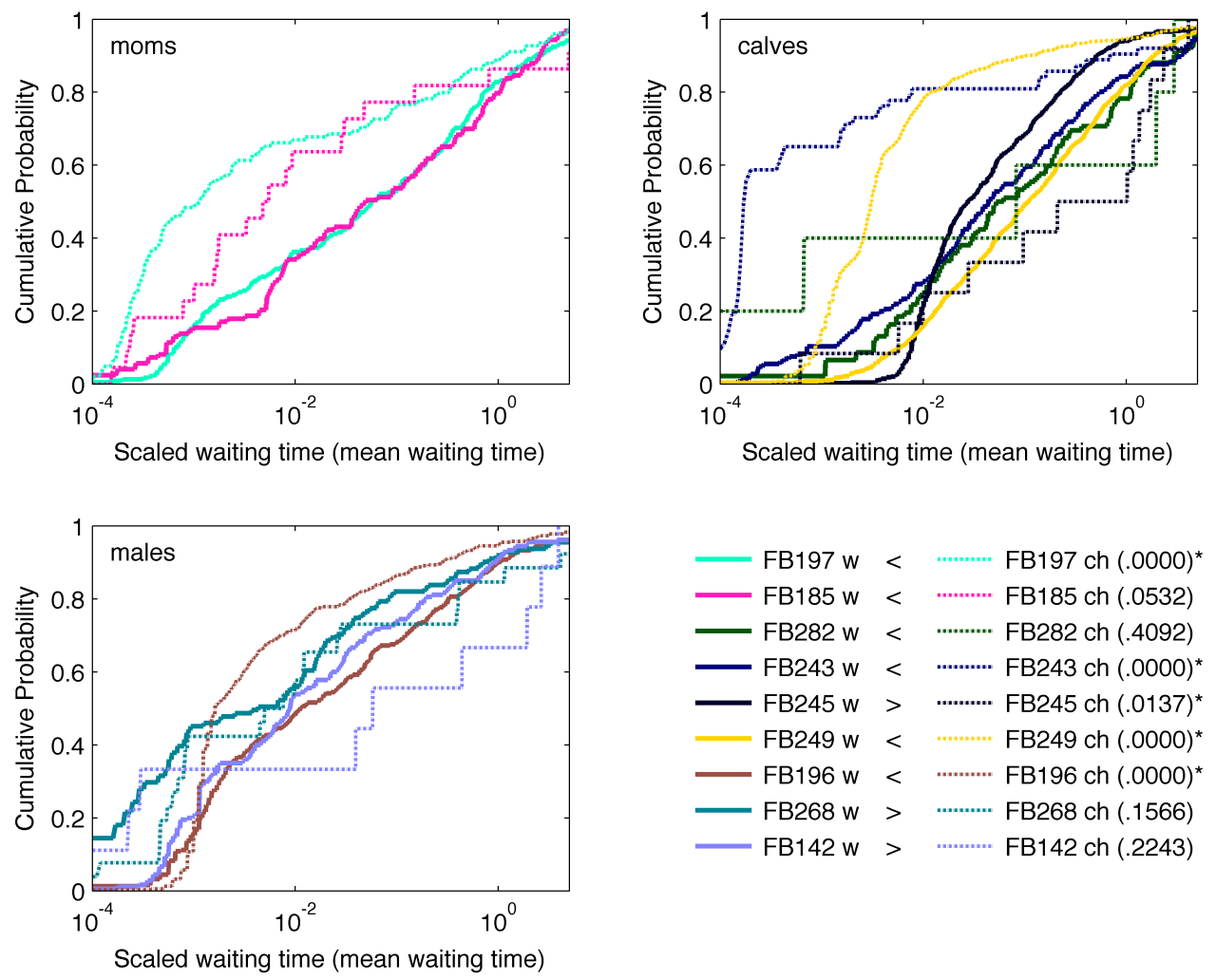

Figure 2.6: Within-animal pairwise comparisons of non-signature whistle (w) and chirp (ch) scaled waiting time distributions. Animals separated into mothers (upper left), calves (upper right) and males (lower left). Each line of the legend shows which curve was greater at the KolmogorovSmirnov statistic test point $(>/<)$ and the p-value is shown in parentheses for a 2-sample test. 
Of the nine animals with sufficient waiting times to compare non-signature whistles and chirps, five showed significantly different shapes when tested with a 2-sample Kolmogorov-Smirnov test. Of those that were significantly different, in four out of five cases chirps showed a relatively more clustered distribution than non-signature whistles.

\subsubsection{Discussion II: waiting time distributions}

Scaling within-animal waiting times allowed me to further characterize and compare the timing of different acoustic cues and their use by the respective demographic classes. I found a wide spread of waiting times, ranging from less than a second to several hours. Once scaled, the shape of the waiting time curves also significantly differed by cuetype within animals, with a strong trend for signature whistles to be clustered relatively closer than non-signature whistles and a weaker trend of chirps grouped tighter than non-signature whistles (Fig 2.4, 2.6).

In one of the few other studies focusing on signature whistle intervals in wild dolphins, Janik et al. (2013) reported that groups of whistles separated by 1-10s were more likely to be signature whistles than those separated by waiting times less than $1 \mathrm{~s}$ or greater than 10s. Although they did not scale to control for base rate sensitivity, like the distributions described in this chapter, their results support the hypothesis that different types of vocalizations are clustered differently. The goal of this chapter is not to evaluate their SigID hypothesis for identifying signature whistles from single hydrophone recordings of groups of wild dolphins. However, for comparison, Table 2.3 matched the signature whistle loop filtering of signature whistle distributions and timing categories used by Janik et al. (2013). 


\begin{tabular}{c|c} 
Inter-SW-Interval & Percent \\
\hline \hline$<1 \mathrm{~s}$ & $32.42 \%$ \\
\hline $1-5 \mathrm{~s}$ & $37.44 \%$ \\
\hline $5-10 \mathrm{~s}$ & $7.43 \%$ \\
\hline$>10 \mathrm{~s}$ & $22.71 \%$ \\
\hline
\end{tabular}

Table 2.3: Percent of inter-signature whistle-intervals described in this chapter filtered and matched according to Janik, et al. (2013)

The largest group of signature whistles analyzed in this chapter (44.87\%) had waiting times between 1 and $10 \mathrm{~s}$. Fewer had intervals less than $1 \mathrm{~s}(32.42 \%)$, and $22.71 \%$ of signature whistles had intervals greater than 10s. These results are similar to those reported by Janik et al. (2013); however, while they found that the majority of signature whistles were separated by $5-10$ s intervals, these boundaries only encompassed $7.43 \%$ of signature whistles analyzed in this chapter. The goal of their study was to create a conservative way to reliably identify signature whistles without tags. Thus, even though the spread of intervals found in this chapter do not overlap entirely, it does not necessarily undermine their method, rather it again highlights the usefulness of on-animal tags for recording a focal animal's highly variable vocal repertoire.

The curves for signature whistles and the ones for chirps (Fig 2.5) were significantly different within animals, but the comparison was less consistently directional than between signature and non-signature whistles: the clustering between cuetypes differed for each animal, but one category was not consistently tighter than the other as in Figure 2.4. Splitting cuetypes by demographic classes (Fig 2.3) showed significant differences between the shapes of non-signature whistle distributions, but there was insufficient evidence to reject the null hypothesis that the waiting time of other cue classes differed by animal type. This difference may support the idea that the non- 
signature whistle group may actually be composed of several different categories that might have different functions.

The distributions of the buzzes were particularly similar, with the curves almost super-imposed on each other, suggesting that they were perhaps used in the same way between animals. It may be that animals foraging within one community are solving the same problem with the same technique. A similar trend was true for chirps, suggesting that they were also used in similar ways between animals. It is important to note that despite similarities for buzzes and chirps, in general the individual variability within animals may obscure our ability to compare across demographics, limiting our ability to assess potential differences in demographic use once averaged.

The general story is one of individual variability, with significant within-animal differences between the shape of the waiting time distributions for signature and nonsignature whistles and chirps. These differences in waiting time distribution shape between call types are consistent with a hypothesis that these acoustic cues may be serving different functions.

\subsubsection{Results III: response latency and inter-animal calling behavior}

To compare the influence of one animal's calling on its partner's acoustic behavior, I first looked at the signature whistle production that follows a partner's signature whistle. Then, I more generally considered the inter-signature whistle waiting time distributions within animals and between partners before testing whether or not an animal is more likely to make a signature whistle within five seconds of its partner's signature whistle. Going one step deeper, I look into these between-animal latency 
distributions to test whether there are timing asymmetries that might reflect hierarchies within partnerships.

Table 2.4 shows the proportion of an animal's signature whistles that were followed within 20s by its partner's signature whistle.

\begin{tabular}{c|c|r|r|r|r} 
ID & Sex & Age & SW Produced & Followed in 20s & Proportion \\
\hline \hline FB33 & F & 31 & 6 & 0 & 0.00 \\
\hline FB282 & M & 5 & 30 & 0 & 0.00 \\
\hline FB196 & M & 16 & 147 & 25 & 0.17 \\
\hline FB268 & M & 21 & 27 & 12 & 0.44 \\
\hline FB276 & M & 22 & 29 & 10 & 0.34 \\
\hline FB142 & M & 22 & 166 & 31 & 0.19 \\
\hline FB197 & F & 11 & 219 & 63 & 0.29 \\
\hline FB243 & F & 2 & 545 & 155 & 0.28 \\
\hline FB133 & F & 15 & 29 & 19 & 0.66 \\
\hline FB245 & F & 2 & 811 & 29 & 0.04 \\
\hline FB185 & F & 14 & 177 & 162 & 0.92 \\
\hline FB249 & F & 3 & 747 & 215 & 0.29
\end{tabular}

Table 2.4: Signature whistles produced by known animals and the number and proportion of those whistles that were followed by the simultaneouslytagged partner producing its own signature whistle within 20s. Partner couplets are shaded the same color.

Figure 2.7 plots this across-animal latency distribution for all animals and the withinanimal signature whistle waiting time distribution described earlier. 


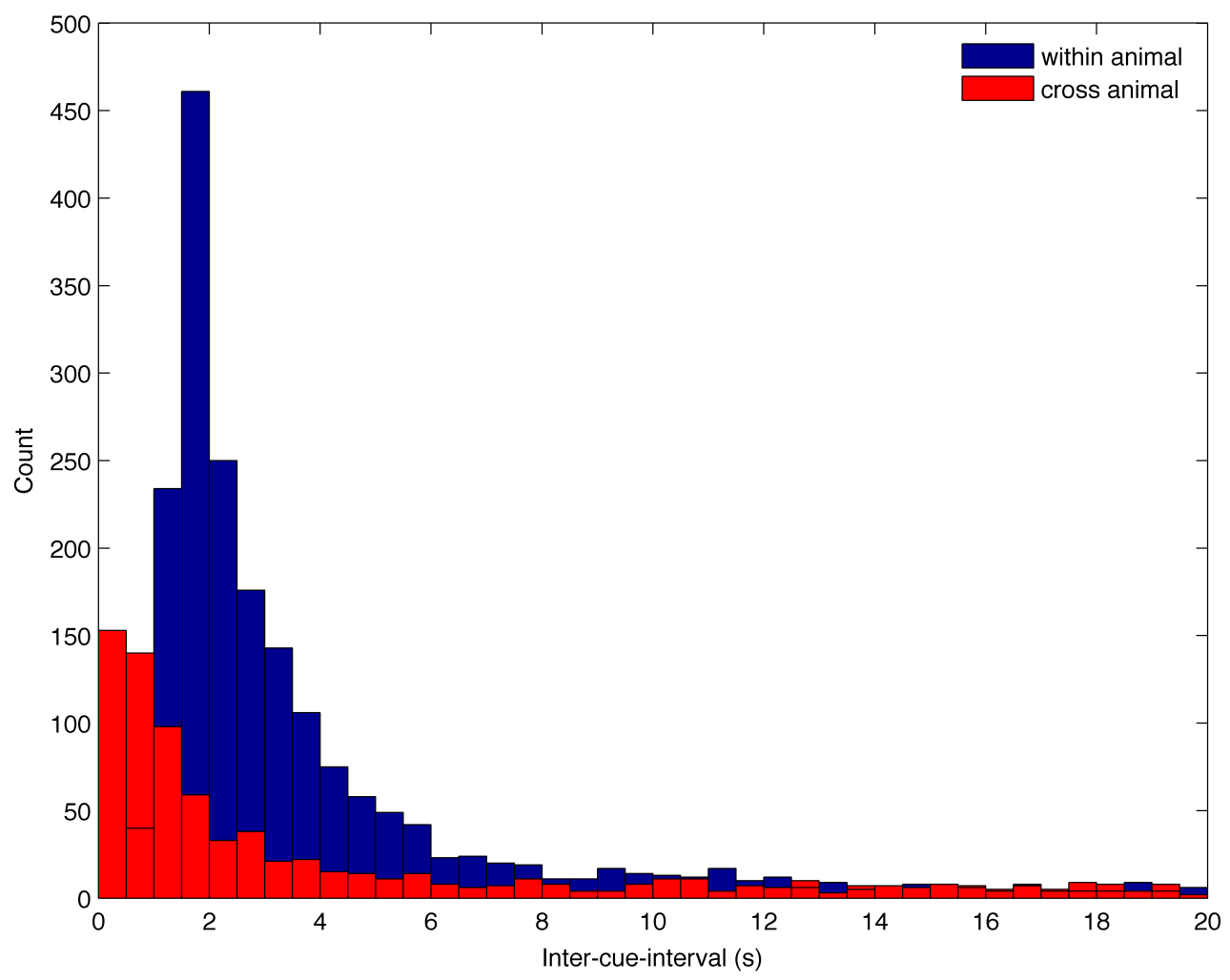

Figure 2.7: Histogram showing the distributions of inter-signature whistle waiting times for all animals within single individuals (blue) and between one individual producing a signature whistle and its partner responding with their own signature whistle (red).

This characterization uses absolute time and shows both that most signature whistle waiting times are less than five seconds (see Table 2.3) and that the intervals across animals are generally shorter than the ones within animals. The cutoff of five seconds was used for the subsequent response latency investigation.

Considering signature whistle production between partners, I found that bottlenose dolphins were significantly more likely than expected by chance to produce signature whistles in the five seconds following their partner's signature whistle (Table 2.5). 


\begin{tabular}{c|c|r|r} 
ID & Sex & Age & P-value \\
\hline \hline FB33 & F & 31 & NA \\
\hline FB282 & $\mathrm{M}$ & 5 & NA \\
\hline FB196 & $\mathrm{M}$ & 16 & .0001 \\
\hline FB268 & $\mathrm{M}$ & 21 & .0001 \\
\hline FB276 & $\mathrm{M}$ & 22 & .002 \\
\hline FB142 & $\mathrm{M}$ & 22 & .0023 \\
\hline FB197 & $\mathrm{F}$ & 11 & $<.0001$ \\
\hline FB243 & $\mathrm{F}$ & 2 & .0016 \\
\hline FB133 & $\mathrm{F}$ & 15 & .1515 \\
\hline FB245 & $\mathrm{F}$ & 2 & .1373 \\
\hline FB185 & $\mathrm{F}$ & 14 & $<.0001$ \\
\hline FB249 & $\mathrm{F}$ & 3 & .0002
\end{tabular}

Table 2.5: Results for a rotation test on the null hypothesis that there is no effect of a partner producing a signature whistle on a dolphin's own signature whistle production in the following $5 \mathrm{~s}$. Each row shows the pvalue for an animal's signature whistle production following its partner's signature whistle being greater than expected by chance.

FB33 and FB282 had no signature whistles within five seconds of each other, so they were not included in the analysis. Eight of the remaining ten animals showed a strong effect of their partner's signature whistle increasing their own signature whistle production within five seconds.

Looking within the subset of intervals that showed signature whistle exchanges within five seconds, Figure 2.8 compares the response latency distributions for partnered animals. 

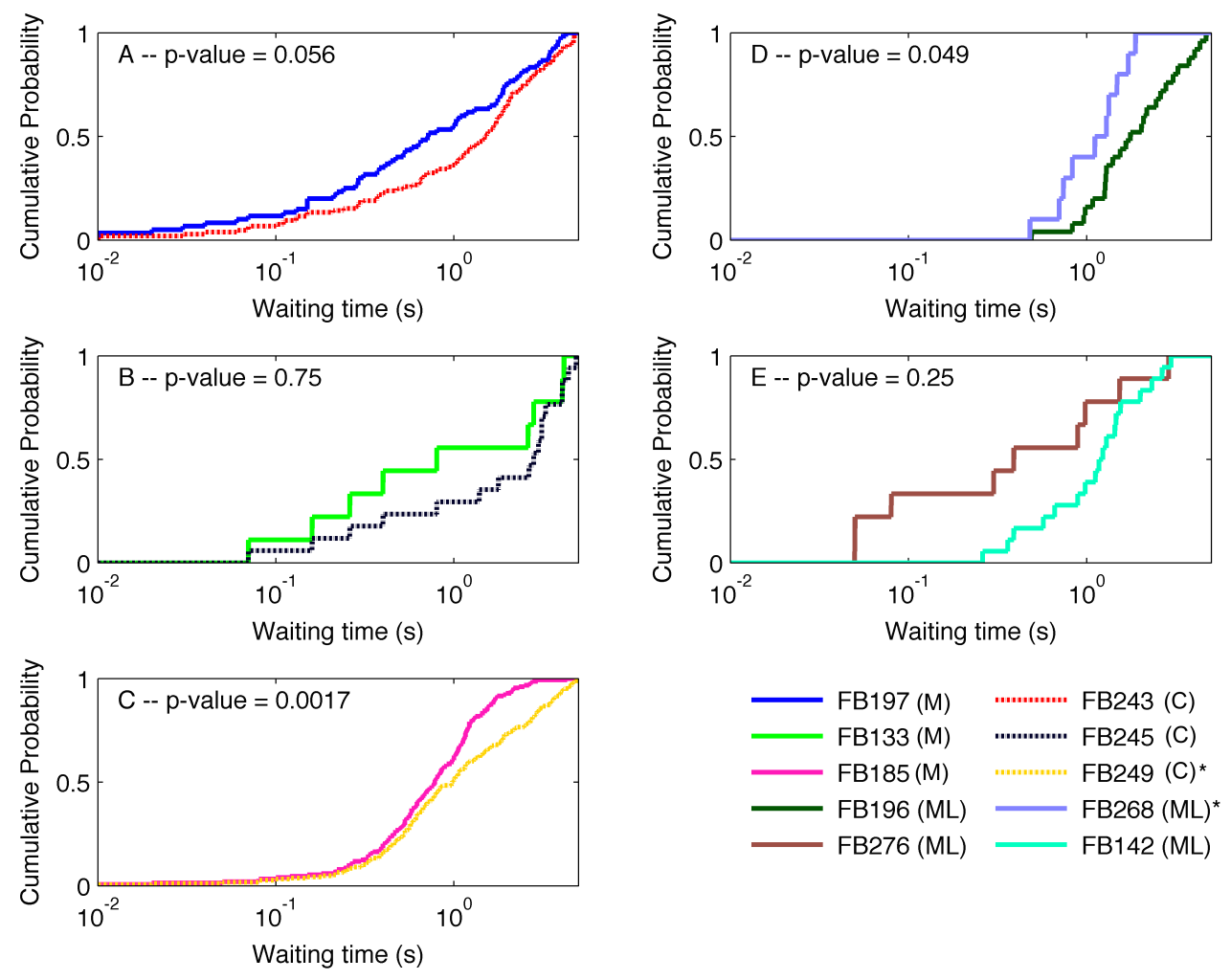

Figure 2.8: Signature whistle response latency distribution within animal pairs (mother (M)-calf $(\mathrm{C})$ on the left and male-male (ML) on the right). The p-value for a 2-sample Kolmogorov-Smirnov test for that pair's distribution is shown in the upper left hand corner of each plot.

I found significant differences between FB196 and FB268, a pair of allied males ( $\mathrm{p}=$ $0.049)$ and between FB185 and FB249, a mother-calf pair $(p=0.0002)$. Another mothercalf pair, FB197 and FB243, also approached significance $(\mathrm{p}=0.056)$. Comparisons were done using a two-sample Kolmogorov-Smirnov test on the unscaled response latencies that were less than five seconds. Repeating the comparison for non-signature whistles and chirps, I found no significant differences within partners. 


\subsubsection{Discussion III: response latency and inter-animal calling behavior}

Rather than describing the characteristic timing of a particular cue, the distribution of response latencies for signature whistles tells us something about the influence of one animal on the calling behavior of another. Here, I found that animals were more likely than expected to produce a signature whistle in the five seconds following their partner's signature whistle. Vocal exchanges where a receiver responds to a produced signal by replying with its own signal have been reported in many animals ranging from primates (Sugiura, 1993, 1998; Miller et al., 2009) to song birds (Krebs et al., 1981) to odontocetes (Nakahara \& Miyazaki, 2011; Quick \& Janik, 2012; King et al., 2014). Thus, the observed increase in probability of a signature whistle is consistent with the hypothesis that animals are responding to each other's signature whistles by producing their own signature whistles. This increase could support a model of contact calling where one animal is attempting to elicit a behavioral change, and the partner is requested to respond.

Using playback experiments with captive dolphins, King et al. (2014) found that once an animal produced its signature whistle, a computer-simulated signature whistle was most likely to elicit a response if it followed in less than one second. A response interval where the animal waits for a reply is consistent with the difference between the cross- and within-distributions shown in Figure 2.7. The cross-animal responses are generally shorter than the within-animal waiting time, suggesting that the longer withinanimal waiting time may be allowing for the possibility of a partner's response.

Looking deeper into the vocal exchanges where animals responded to a signature whistle with their own signature whistle within five seconds, I found asymmetries in the 
distribution of time prior to this response (response latency). This asymmetry is reported to vary with individual identity and affiliative relationships of the animals (Rendall et al., 1996; Soltis et al., 2005) and likely behavioral state as well. In our dataset, two out of the five pairs showed significant differences between their response latency distributions for signature whistles at a 5\% level, and another mother-calf pair showed a strong similar trend $(\mathrm{p}=0.056)$. The data from the mothers and calves exhibit a pattern towards a much larger proportion of the calf signature whistles following closely after a mother's signature whistle than vice versa, even once differences in call rate are taken into account. This contrast may reflect an asymmetry in the relationship. Were this true, the shape of this distribution might change for both the mother and the calf as the calf ages. Perhaps a pregnant mother would become even less responsive as she encourages a calf to leave. The shape of the response latency distribution might also be an indicator of hierarchy, particularly between allied males in a pair. I predict that more dominant animals might command a quicker reply than less dominant ones. Although we see this difference in one out of two male pairs, at present we lack the sample size to accurately assess age effects between mothers and calves or dominance between males.

\subsubsection{Results IV: individually-specific mark recaptures}

Unmodelled heterogeneity in the probability of detecting an animal can pose problems for simple mark recapture abundance estimates. I explored this variability in signature whistle probability of detection in two ways. First, to get a measure of confidence for our ability to detect a specific animal given a certain amount of time within range of a passive acoustic monitoring system, I explored the relationship between recording time and the probability of detecting a signature whistle for each tag. Second, I 
considered the effect of inter-individual variability on detection probability in an eightminute listening window by creating likelihood functions for the number of animals present that create different numbers of detected signature whistles.

Figure 2.9 shows the estimated probability of detecting an animal's signature whistle at increasing listening times for each individual dolphin tagged.

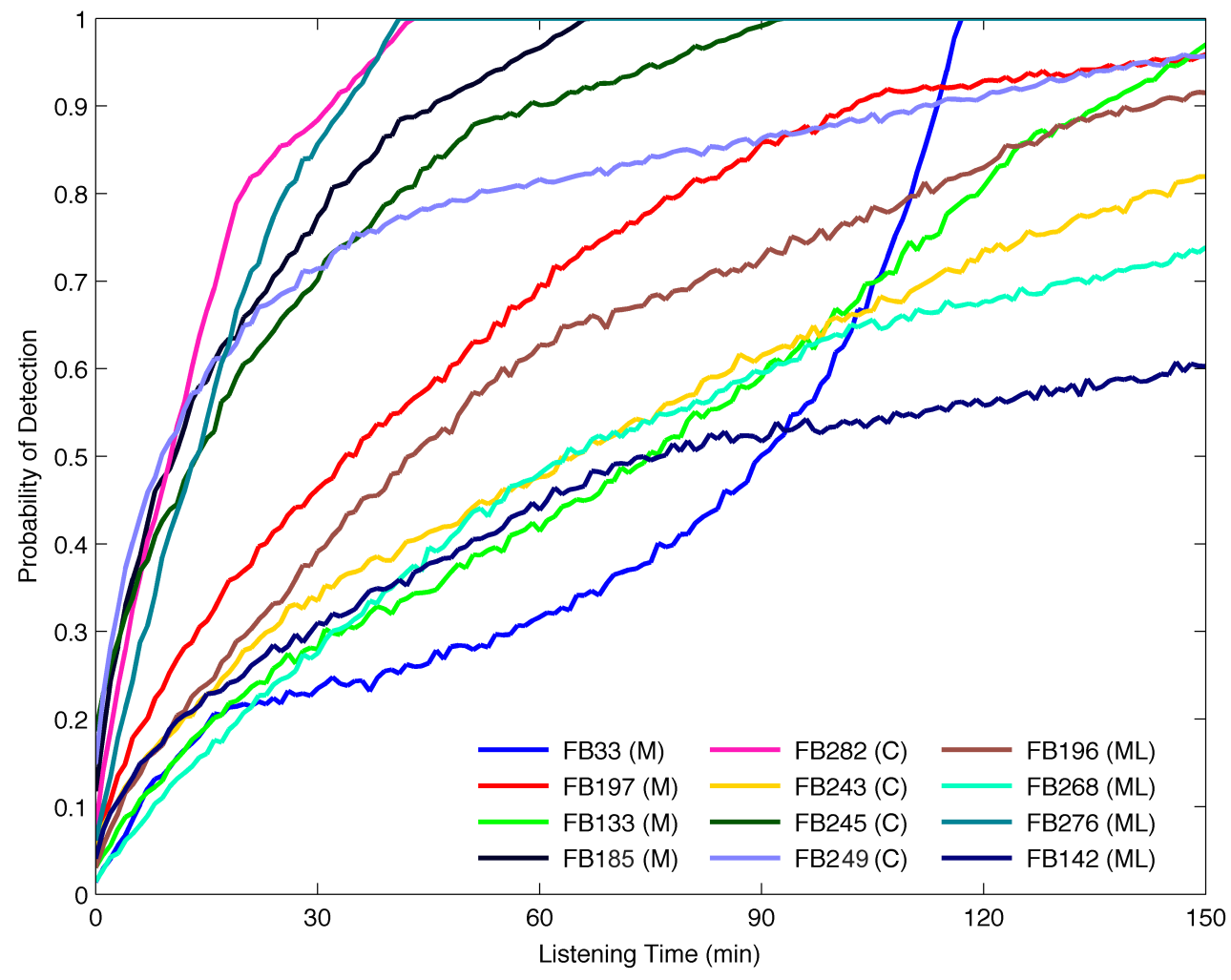

Figure 2.9: Probability of detecting an animal's signature whistle given a length of recording from that animal. Legend is organized by mothers $(\mathrm{M})$, calves (C) and males (ML).

Based on these curves, it might take anywhere from minutes to hours to detect a signature whistle from a particular animal. Table 2.6 shows estimates of required recording time (in minutes) for $25,50,75,95$ and $100 \%$ percent chance of detecting at least one signature whistle from an animal. 


\begin{tabular}{|c|c|c|c|c|c|c|c|}
\hline ID & Sex & Age & $\begin{array}{l}25 \% \\
\text { (min) }\end{array}$ & $\begin{array}{c}50 \% \\
(\mathrm{~min})\end{array}$ & $\begin{array}{r}75 \% \\
\text { (min) }\end{array}$ & $\begin{array}{l}95 \% \\
(\mathrm{~min})\end{array}$ & $\begin{array}{l}100 \% \\
(\mathrm{~min})\end{array}$ \\
\hline FB33 & $\mathrm{F}$ & 31 & 40 & 91 & 109 & 117 & 118 \\
\hline FB282 & $\mathrm{M}$ & 5 & 5 & 12 & 19 & 39 & 44 \\
\hline FB196 & $\mathrm{M}$ & 16 & 18 & 44 & 95 & 168 & 190 \\
\hline FB268 & $\mathrm{M}$ & 21 & 28 & 65 & 156 & 231 & 245 \\
\hline FB276 & $\mathrm{M}$ & 22 & 7 & 15 & 25 & 39 & 42 \\
\hline FB142 & $\mathrm{M}$ & 22 & 21 & 78 & 230 & 300 & 315 \\
\hline FB197 & $\mathrm{F}$ & 11 & 11 & 35 & 70 & 141 & 190 \\
\hline FB243 & $\mathrm{F}$ & 2 & 19 & 64 & 127 & 192 & 226 \\
\hline FB133 & $\mathrm{F}$ & 15 & 25 & 76 & 113 & 148 & 157 \\
\hline FB245 & $\mathrm{F}$ & 2 & 3 & 15 & 37 & 79 & 94 \\
\hline FB185 & $\mathrm{F}$ & 14 & 4 & 12 & 29 & 57 & 68 \\
\hline FB249 & $\mathrm{F}$ & 3 & 3 & 10 & 36 & 143 & 198 \\
\hline
\end{tabular}

Table 2.6: Continuous listening time in minutes required in order to detect an animal's signature whistle a given percent of the time.

To detect an animal by passive acoustic monitoring based on its signature whistle, that dolphin must produce one while in range of the recording system, be it an array or a hydrophone. Since dolphins are generally moving, I used eight minutes to reflect a hypothetical recording window based on an average dolphin travel speed of $2 \mathrm{~ms}^{-1}$ and an active space radius estimate of 487m (Quintana-Rizzo et al., 2006).

Figure 2.10 propagates this variability to show the likelihood functions for the number of animals producing a given number of different signature whistles detected in an eight-minute window, assuming that our tagged dolphins are a representative sample of the population. 


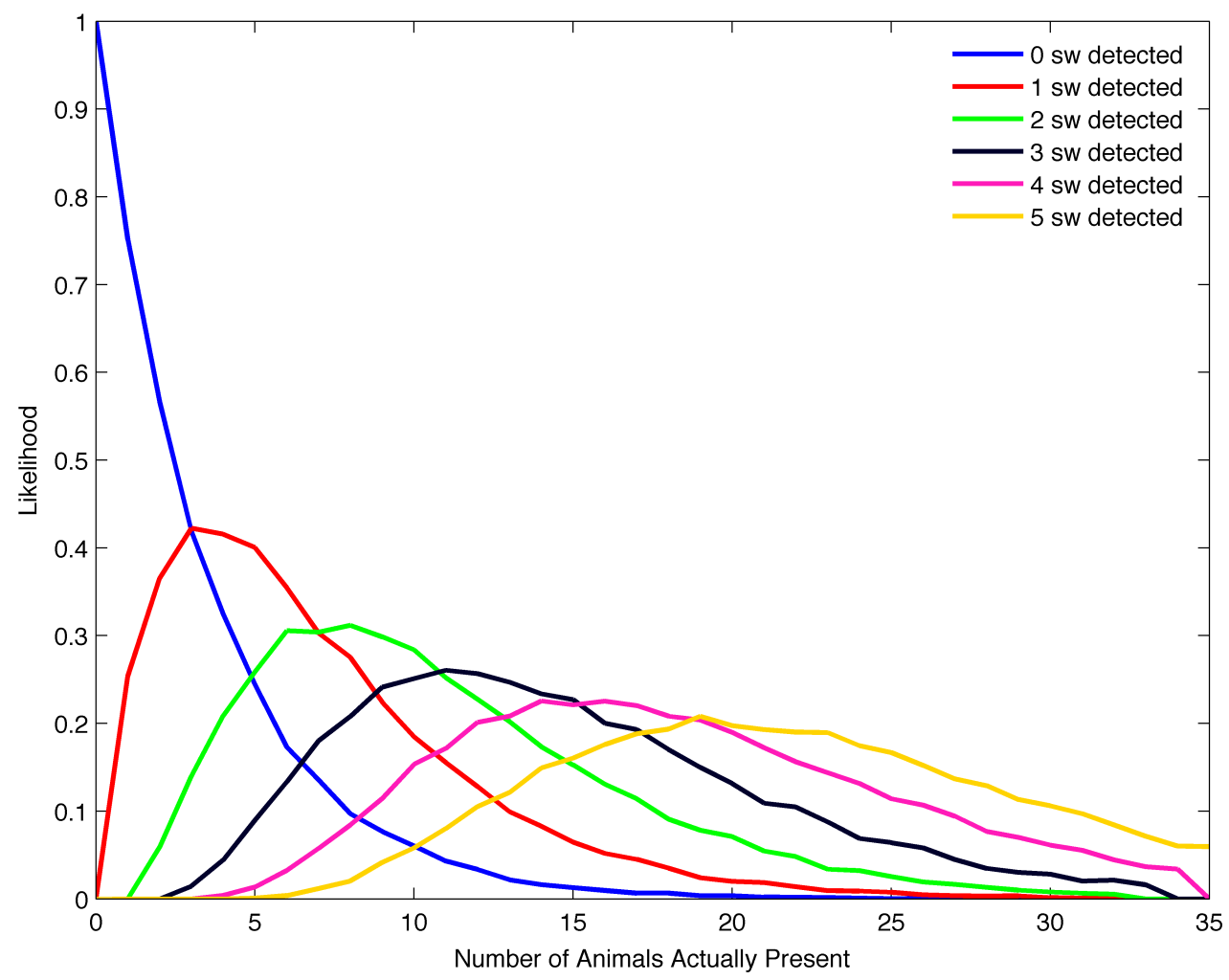

Figure 2.10: Likelihood curves for estimating the number of individuals present, given the number of signature whistles (sw) detected over $8 \mathrm{~min}$. Each curve represents the spread of individuals that could produce a particular number of detected whistles. Note that the number of animals must be greater than or equal to the number of different detected signature whistles.

\subsubsection{Discussion IV: individually-specific mark recaptures}

Passive acoustic monitoring provides an affordable and practical alternative to visual surveys for estimating animal abundance. Given the individually-distinctive nature of bottlenose dolphin signature whistles (Janik \& Sayigh, 2013) and the rarity of signature whistle copying by other animals (King et al., 2013), signature whistles are good candidates for acoustic mark-recapture studies. Sarasota Bay, with its known 
population, would be uniquely suited to ground-truth such a passive acoustic monitoring technique.

A key assumption of many simple mark-recapture methods is that the probability of detection is same across animals. This chapter's results suggest that the inter-animal variability in the probability of producing a signature whistle may strain this assumption and could introduce uncertainty that would carry over into abundance estimates. This variability in probability of detection may stem from the clustering of signature whistles, with bottlenose dolphins routinely going through long periods of silence at unpredictable intervals. This uncertainty is reflected in the spread shape of the likelihood functions for different numbers of detected signature whistles. The shape flattens as the number of detected signature whistles increases, suggesting that a wider range of animals present are likely to be recorded producing five signature whistles than one signature whistle, and it may be challenging to use these curves to estimate population size.

This chapter assumed that the sample of tagged animals is representative of the entire population. The accuracy of this assumption is uncertain; however, these are the first data of their kind, so it is not currently possible to test.

\subsection{CONCLUSION}

The Sarasota health assessments have provided a successful opportunity for deploying suction-cup tags on wild bottlenose dolphins, yielding novel insights into the rate and timing differences between acoustic cue types and demographic classes.

The data presented in this chapter describe vocal rates that were highly variable between individuals, but differences between the shapes of the waiting time distributions 
for different types of acoustic signals are consistent with the idea that signature whistles, non-signature whistles and chirps are serving different functions. In particular, signature whistles show a strong trend of being clustered tighter than non-signature whistles. One potential interpretation for this tight clustering would be that signature whistles may have a contact call function that escalates a demand from a partner who is not responding. The relatively less-clustered distribution of non-signature whistles may reflect the possibility that this category could be composed of several different subcategories. In terms of responses to signature whistles, dolphins were more likely to produce a signature whistle within five seconds of their partner's signature whistling, supporting the idea that they may be using signature whistles as responses. Moreover, significant differences in the latency of this response exist between some partners, consistent with the idea of an asymmetry in the relationship. In terms of mark-recapture, I found heterogeneity in the probability of detection that might stretch a key assumption of mark-recapture methods if not taken into account in the modeling. Using this variability to simulate likelihood curves showed that a given number of detected signature whistles are likely to be produced by a range of animals.

Overall, simultaneous tagging is a powerful new tool for studying the behavior of bottlenose dolphins. The four years of fieldwork described in this chapter have for the first time provided an on-animal acoustic window into the behavior of wild freeswimming bottlenose dolphins where we can both capture almost every focal vocalization and accurately attribute caller identity. Although the health assessments represent a special deployment case, the results described in this chapter suggest that if 
pole-tagging of other small delphinids becomes possible, it will reveal useful insights into their vocal behavior and communication. 


\subsection{REFERENCES}

Beeman, K. (1998). Digital signal analysis, editing, and synthesis. In S. L. Hopp, M. J. Owren \& C. S. Evans (Eds.), Animal acoustic communication (pp. 59-103). New York: Springer.

Borchers, D. (2012). A non-technical overview of spatially explicit capture-recapture models. Journal of Ornithology, 152(2), 435-444.

Bowyer, R. T., \& Kitchen, D. W. (1987). Sex and age-class differences in vocalizations of Roosevelt elk during rut. American Midland Naturalist, 225-235.

Bradbury, J. W., \& Vehrenkamp, S. L. (1998). Principles of acoustic communication. Sunderland, MA: Sinauer Associates.

Caldwell, M. C., \& Caldwell, D. K. (1970). Etiology of the chirp sounds emitted by the Atlantic bottlenosed dolphins: a controversial issue. Underwater Naturalist, 6(3), 6-8 and 43.

Caldwell, M. C., Caldwell, D. K., \& Tyack, P. L. (1990). Review of the signature-whistle hypothesis for the Atlantic bottlenose dolphin. In S. Leatherwood \& R. R. Reeves (Eds.), The Bottlenose Dolphin (pp. 199-234): New York: Academic Press.

Cook, M. L., Sayigh, L. S., Blum, J. E., \& Wells, R. S. (2004). Signature-whistle production in undisturbed free-ranging bottlenose dolphins (Tursiops truncatus). Proceedings of the Royal Society B: Biological Sciences, 271(1543), 1043-1050.

Cvikel, N., Berg, K. E., Levin, E., Hurme, E., Borissov, I., Boonman, A., . . Yovel, Y. (2015). Bats Aggregate to Improve Prey Search but Might Be Impaired when Their Density Becomes Too High. Current Biology.

David, H. T. (1958). A three-sample Kolmogorov-Smirnov test. The Annals of Mathematical Statistics, 842-851.

Deruiter, S. L., \& Solow, A. R. (2008). A rotation test for behavioural point-process data.

Evans, C. S., Evans, L., \& Marler, P. (1993). On the meaning of alarm calls: functional reference in an avian vocal system. Animal Behaviour, 46(1), 23-38.

Ghazanfar, A. A., Smith-Rohrberg, D., Pollen, A. A., \& Hauser, M. D. (2002). Temporal cues in the antiphonal long-calling behaviour of cottontop tamarins. Animal Behaviour, 64(3), 427-438.

Green, S. M. (1981). Sex differences and age gradations in vocalizations of Japanese and lion-tailed monkeys (Macaca fuscata and Macaca silenus). American Zoologist, 21(1), 165-183.

Herman, L. M., \& Tavolga, W. N. (1980). The communication systems of cetaceans. In L. M. Herman (Ed.), Cetacean behavior: Mechanisms and functions (pp. 149209). New York: John Wiley \& Sons, Inc.

Janik, V. M. (2009). Acoustic communication in delphinids. In M. Naguib, V. Janik, N. Clayton \& K. Zuberbuhler (Eds.), Advances in the Study of Behavior (Vol. 40, pp. 123-157): Academic Press.

Janik, V. M., King, S. L., Sayigh, L. S., \& Wells, R. S. (2013). Identifying signature whistles from recordings of groups of unrestrained bottlenose dolphins (Tursiops truncatus). Marine Mammal Science, 29, 1-14.

Janik, V. M., \& Sayigh, L. S. (2013). Communication in bottlenose dolphins: 50 years of signature whistle research. Journal of Comparative Physiology A, 199(6), 479489. 
Janik, V. M., \& Slater, P. J. (1998). Context-specific use suggests that bottlenose dolphin signature whistles are cohesion calls. Animal Behaviour, 56, 829-838.

Johnson, M., Jensen, F. H., Miller, P., Madsen, P. T., de Soto, N. A., \& Tyack, P. L. (2015). Measuring the separation distance and relative location of animals using sound recording tags. Behavioral Ecology and Sociobiology, Submitted.

Johnson, M. P., Aguilar de Soto, N., \& Madsen, P. (2009). Studying the behaviour and sensory ecology of marine mammals using acoustic recording tags: a review. Marine Ecology Progress Series, 395, 55-73.

Johnson, M. P., \& Tyack, P. L. (2003). A digital acoustic recording tag for measuring the response of wild marine mammals to sound. IEEE Journal of Oceanic Engineering, 28(1), 3-12.

King, S. L., Harley, H. E., \& Janik, V. M. (2014). The role of signature whistle matching in bottlenose dolphins, Tursiops truncatus. Animal Behaviour, 96, 79-86.

King, S. L., Sayigh, L. S., Wells, R. S., Fellner, W., \& Janik, V. M. (2013). Vocal copying of individually distinctive signature whistles in bottlenose dolphins. Proceedings of the Royal Society B: Biological Sciences, 280(1757).

Kitchen, D. M. (2004). Alpha male black howler monkey responses to loud calls: effect of numeric odds, male companion behaviour and reproductive investment. Animal Behaviour, 67(1), 125-139.

Krams, I., Krama, T., Freeberg, T. M., Kullberg, C., \& Lucas, J. R. (2012). Linking social complexity and vocal complexity: a parid perspective. Philosophical Transactions of the Royal Society B: Biological Sciences, 367(1597), 1879-1891.

Krebs, J. R., Ashcroft, R., \& Van Orsdol, K. (1981). Song matching in the Great Tit Parus major. Animal Behaviour, 29(3), 918-923.

Link, W. A. (2003). Nonidentifiability of population size from capture-recapture data with heterogeneous detection probabilities. Biometrics, 59(4), 1123-1130.

Macedonia, J. M. (1990). What is communicated in the antipredator calls of lemurs: evidence from playback experiments with ringtailed and ruffed lemurs. Ethology, 86(3), 177-190.

Macfarlane, N. B. W. (2015). Tagging wild cetaceans: investigating the balance between more and less invasive techniques. (SM), Massachusetts Institute of Technology.

Manser, M. B. (2001). The acoustic structure of suricates' alarm calls varies with predator type and the level of response urgency. Proceedings of the Royal Society B:

Biological Sciences, 268(1483), 2315-2324.

Marques, T. A., Thomas, L., Martin, S. W., Mellinger, D. K., Ward, J. A., Moretti, D. J., . . . Tyack, P. L. (2013). Estimating animal population density using passive acoustics. Biological Reviews, 88(2), 287-309.

Mellinger, D. K., Stafford, K. M., Moore, S., Dziak, R. P., \& Matsumoto, H. (2007). Fixed passive acoustic observation methods for cetaceans. Oceanography, 20(4), 36.

Miller, C. T., Beck, K., Meade, B., \& Wang, X. (2009). Antiphonal call timing in marmosets is behaviorally significant: interactive playback experiments. Journal of Comparative Physiology A, 195(8), 783-789.

Miller, P. J. O., Shapiro, A. D., Tyack, P. L., \& Solow, A. R. (2004). Call-type matching in vocal exchanges of free-ranging resident killer whales, Orcinus orca. Animal Behaviour, 67(6), 1099-1107. 
Nakahara, F., \& Miyazaki, N. (2011). Vocal exchanges of signature whistles in bottlenose dolphins (Tursiops truncatus). Journal of ethology, 29(2), 309-320.

Quick, N. J., \& Janik, V. M. (2012). Bottlenose dolphins exchange signature whistles when meeting at sea. Proceedings of the Royal Society B: Biological Sciences, 279, 2539-2545.

Quintana-Rizzo, E., Mann, D. A., \& Wells, R. S. (2006). Estimated communication range of social sounds used by bottlenose dolphins (Tursiops truncatus). Journal of the Acoustical Society of America, 120(3), 1671-1683.

Rendall, D., Rodman, P. S., \& Emond, R. E. (1996). Vocal recognition of individuals and kin in free-ranging rhesus monkeys. Animal Behaviour, 51(5), 1007-1015.

Schel, A. M., Candiotti, A., \& Zuberbühler, K. (2010). Predator-deterring alarm call sequences in Guereza colobus monkeys are meaningful to conspecifics. Animal Behaviour, 80(5), 799-808.

Schneider, K., Baird, R. W., Dawson, S., Visser, I., \& Childerhouse, S. (1998). Reactions of bottlenose dolphins to tagging attempts using a remotely-deployed suction-cup tag. Marine Mammal Science, 14(2), 316-324.

Scott, M., Wells, R., \& Irvine, A. B. (1990). A long-term study of bottlenose dolphins on the West coast of Florida. In S. Leatherwood \& R. R. Reeves (Eds.), The bottlenose dolphin (pp. 235-244). San Diego: Academic Press.

Sherman, P. W. (1977). Nepotism and the evolution of alarm calls. Science, 197(4310), 1246-1253.

Soltis, J., Leong, K., \& Savage, A. (2005). African elephant vocal communication I: antiphonal calling behaviour among affiliated females. Animal Behaviour, 70(3), 579-587.

Sugiura, H. (1993). Temporal and acoustic correlates in vocal exchange of coo calls in Japanese macaques. Behaviour, 124(3), 207-225.

Sugiura, H. (1998). Matching of acoustic features during the vocal exchange of coo calls by Japanese macaques. Animal Behaviour, 55(3), 673-687.

Templeton, C. N., Greene, E., \& Davis, K. (2005). Allometry of alarm calls: blackcapped chickadees encode information about predator size. Science, 308(5730), 1934-1937.

Thomas, L., Jarvis, S., DiMarzio, N., Moretti, D., Marques, T. A., Dunn, C., . . Tyack, P. (2012). Passive acoustic density estimation of sperm whales in the Tongue of the Ocean, Bahamas. Marine Mammal Science, 28(4), E444-E455.

Townsend, S. W., Hollén, L. I., \& Manser, M. B. (2010). Meerkat close calls encode group-specific signatures, but receivers fail to discriminate. Animal Behaviour, 80(1), 133-138.

Tyack, P. L. (2000). Functional aspects of cetacean communication. In J. Mann, R. C. Connor, P. L. Tyack \& H. Whitehead (Eds.), Cetacean Societies: Field studies of whales and dolphins (pp. 270-307). Chicago: University of Chicago Press.

Tyack, P. L., \& Clark, C. W. (2000). Communication and acoustic behavior of dolphins and whales Hearing by whales and dolphins (pp. 156-224): Springer.

van der Hoop, J. M., Fahlman, A., Hurst, T., Rocho-Levine, J., Shorter, K. A., Petrov, V., \& Moore, M. J. (2014). Bottlenose dolphins modify behavior to reduce metabolic effect of tag attachment. The Journal of Experimental Biology, 217(23), 42294236. 
Wells, R. S. (1991). The role of long-term study in understanding the social structure of a bottlenose dolphin community. In K. Pryor \& K. Norris (Eds.), Dolphin societies: discoveries and puzzles (pp. 199-225). Berkeley: University of California Press.

Wells, R. S. (2003). Dolphin social complexity: lessons from long-term study and lifehistory. In F. B. M. De Waal \& P. L. Tyack (Eds.), Animal social complexity: intelligence, culture, and individualized societies. Cambridge: Harvard University Press.

Wells, R. S. (2009). Learning from nature: bottlenose dolphin care and husbandry. Zoo Biology, 28(6), 635-651.

Wells, R. S., Rhinehart, H. L., Hansen, L. J., Sweeney, J. C., Townsend, F. I., Stone, R., . . Rowles, T. K. (2004). Bottlenose dolphins as marine ecosystem sentinels: developing a health monitoring system. EcoHealth, 1(3), 246-254.

Wells, R. S., Scott, M., \& Irvine, A. (1987). The social structure of free-ranging bottlenose dolphins. Current Mammalogy, 1, 247-305. 


\section{Chapter 3: A reunion calling function for signature whistles in wild bottlenose dolphins}




\subsection{ABSTRACT}

Animals with stable relationships need mechanisms to stay in touch when separated. Five decades of research suggest that signature whistles are likely candidates for serving this contact calling purpose in bottlenose dolphins. However, difficulties identifying the vocalizing individual and measuring inter-animal distances have hindered tests of call functions among wild dolphins. Moreover, signature whistles almost certainly serve a variety of functions, so that to focus on contact calling, it will be useful to identify contexts where animals need to maintain cohesion. By simultaneously tagging pairs of mothers and calves to look at instances when the animals are separating and reuniting, I can focus on testing specific contact functions of signature whistles. Drawing from the literature, I define three potential kinds of contact call functions for signature whistles, each with its own hypothetical signature whistle distribution during separations and reunions: location monitoring, reunion calls, and identity advertisement calls. To explore these potential functions, this chapter examines the probability of an animal producing a signature whistle at different stages of temporary separation events. Using a binomial logistic regression model, I found that the data are consistent with signature whistles functioning as reunion calls or identity advertisement calls but not for location monitoring during separations and reunions.

\subsection{INTRODUCTION}

In his review of infant crying in primates, Newman (1985) points out that the need for the young of all mammals to suckle creates the need for prolonged contact, which in many species has selected for early development of contact calling. The cry of a human infant functions to alert caregivers when the infant is hungry or distressed, but the most common trigger for crying is being alone, and the most common response being picked up. Newman (1985) reports that many non-human primates produce similar cries, which he calls isolation calls. In species with strong individually-specific social relationships, the isolation call may persist into adulthood, with species-, geographic- and individual-specific markers. Animals with stable social relationships need mechanisms to stay in touch when they are separated. For animals in open habitats, modalities like vision 
might be sufficient, but in low-visibility environments, such as in the jungle or at night, animals often rely on acoustic calls to maintain contact (Bradbury \& Vehrencamp, 1998).

Constraints on visibility are particularly apparent underwater where light attenuates rapidly, and many mobile marine animals have evolved to rely on acoustics for communication. The bottlenose dolphin, Tursiops truncatus, is a prime example of a species that uses sound to mediate interactions (Janik, 2009). Bottlenose dolphins live in fission-fusion societies where they form temporary associations with a variety of different animals but also show strong, stable relationships with certain individuals, in particular between mother and calf or small groups of allied males (Connor et al., 2000). Dolphin calves have a prolonged period of dependency, remaining associated with their mothers for up to 10 years, until the next calf is born (Wells, 1991). Likewise, pairs of male bottlenose dolphins form long and stable alliances with associations that last longer and are almost as strong as the mother-calf bond (Connor et al., 1992; Owen et al., 2002). Managing these complex relationships in a three-dimensional environment with daily individual home ranges of up to $55 \mathrm{~km}^{2}$ and inter-animal separations up to several hundred meters (Connor et al., 2000) would benefit from an individually-distinctive signal for keeping track of each other while separated underwater. Like other toothed whales, dolphins only have a rudimentary olfactory sense, and their auditory system is better developed than their vision (Janik, 2009). Five decades of research have shown that bottlenose dolphins produce learned signature whistles that are individually distinctive and convey individual identity (Janik \& Sayigh, 2013). 


\subsubsection{Signature whistles as candidates for a contact call function}

Signature whistles are often described as associated with contact calling (Caldwell \& Caldwell, 1965; Caldwell et al., 1990), but specific functional hypotheses have not been fully explored. In one of the clearest examples supporting the hypothesis that signature whistles function as contact calls, Janik and Slater (1998) found that captive dolphins primarily produced signature whistles when spontaneously separated from their group members but produced other whistles when together. Work by Smolker et al. (1993) and Watwood et al. (2005) suggests that a similar contact call function during separation might be used by wild dolphins (Tursiops truncatus and Tursiops aduncus). Thus, signature whistles are likely candidates for serving a contact call function. However, difficulties identifying the vocalizing individual and measuring inter-animal distances have hindered tests of these functions among wild bottlenose dolphins.

\subsubsection{Separating a contact call function from stress}

In addition to an association with contact calling, signature whistle rate has been connected to stress. To investigate a potential contact-calling role for signature whistles, this chapter uses separation distance and rate to distinguish between these two contexts. H. C. Esch et al. (2009) used a temporary capture-release situation to show that signature whistle rate appears to increase in stressful situations; the average rate of whistling was $14.3 / \mathrm{min}$ compared to 0.3 whistles/min when undisturbed. Some definitions of stress combine situations when the animal feels discomfort, pain, hunger or cold with situations of isolation. Here I separate these scenarios, and posit that while whistles indicating stress may occur both when animals are close together and separated, contact calls that are unrelated to stress will occur primarily when they are apart. Thus, looking specifically at 
separations and reunions will allow me to focus on a situation where a potential contactcalling role for signature whistles is likely to be expressed.

\subsubsection{Defining contact call functions for signature whistles}

Researchers have reported contact call vocalizations in many different species, but the definition varies from study to study, making generalization complicated. Da Cunha and Byrne (2009) group contact calls into three broad functional categories: keeping contact, regaining lost contact and coordinating group movement. To these three, Kondo and Watanabe (2009) add identity advertisement. These four categories are quite broad, and different species and societies will likely use different aspects of them in different ways. For my purposes, in the fission-fusion societies of bottlenose dolphins where pair-bonded individuals must maintain contact with a partner amongst brief transient associations with other individuals, I will focus on the keeping contact, regaining lost contact and identity advertisement categories.

Drawing from Caine and Stevens (1990), within the "keeping contact" category, I define a location monitoring function as calls that animals make spontaneously when separated to keep track of each other without necessarily leading to a change in cohesion. Examples of this function include red-bellied tamarins using 'slide calls' to monitor the positions of group members (Caine and Stevens 1990) as well as meerkats that emit 'close calls' to monitor other group-members while foraging with their heads down (Townsend et al., 2010).

Within the broad "regaining lost contact" category, I define reunion contact calls to refer to vocalizations that represent a motivation to reunite with group members. Examples of this function are seen in orange-fronted conures, spider monkeys and spear- 
nosed bats, which all make calls that attract conspecifics (Wilkinson \& Boughman, 1998; Vehrencamp et al., 2003; Ramos-Fernández, 2005).

Finally, in terms of identity advertisement, when animals are approaching close enough to exchange resources or face risk of attack, it may be important to confirm identity before reuniting. For example, in colonial breeding birds such as penguins, not only must the parent ensure feeding the correct offspring, but the offspring must be wary of the wrong parent who might attack it (Aubin \& Jouventin, 2002; Searby et al., 2004).

Simultaneously tagging pairs of bottlenose dolphins with acoustic tags provides a unique opportunity to investigate these contact call functions of signature whistles. Not only does the synchronized tagging allow us to attribute which animal is vocalizing with a very high level of confidence, but, for the first time, it also provides a continuous and accurate measure of inter-animal distance, even when animals are underwater and not visible (Johnson et al., 2015).

Here, I use simultaneous tagging techniques to investigate these potential signature whistle functions during temporary separations and reunions. This focus allows me to focus on situations where a mechanism for keeping in touch is most likely to be expressed. If signature whistles serve a monitoring function, I would expect to see spontaneous low rate emission of signature whistles to monitor the position of other group members. This might manifest itself in a certain inter-animal distance threshold above which signature whistle rate is steady and random but with no trend for approach immediately after whistling. For a reunion function, I would expect that if signature whistles represent a motivation to reunite with the paired animal, they would lead to a reduction of inter-animal distance. Thus, I would hypothesize a concentration of 
signature whistles near the maximum point of separation, prior to the animals coming back together. Lastly, if they are used for establishing identity prior to a close approach with another animal, I would expect another concentration of signature whistles in the final phases of approach just prior to the end of the reunion.

\subsection{MeTHODS}

\subsubsection{Field Site}

All data collection was conducted in Sarasota, FL, at the field site introduced in Chapter Two. Since 1970, the resident bottlenose dolphin (Tursiops truncatus) community in Sarasota Bay has been the subject of the world's longest-running study of dolphin populations as part of the Sarasota Dolphin Research Program (Scott et al., 1990; Wells, 1991; Wells, 2003). Currently, the 165 resident dolphins span five generations, with some individuals being observed for more than 40 years (Wells, 2009). Critically for my purposes, the brief capture-releases conducted as part of annual health assessments provide a unique opportunity to deploy suction-cup Digital Acoustic Recording Tags (DTAGV3, Johnson \& Tyack, 2003, Johnson et al., 2009) prior to release.

\subsubsection{Data Collection}

As described in Chapter Two, pairs of closely associated dolphins were simultaneously tagged, either mother-calf pairs or male-male alliances. Animals were followed upon release from a $7 \mathrm{~m}$ vessel to collect behavioral observations and monitor the presence of all other animals in the area. Follow distances were typically $30-80 \mathrm{~m}$. Dolphins were identified each time they surfaced. Focal follows lasted for up to several 
hours and tags were usually retrieved at a later point following release (although some released while the animals were being followed).

\subsubsection{Analysis Method}

Data were analyzed using custom software written in MATLAB (Mathworks, Natick, MA). Two essential parts of data processing are the identification of focal sounds and the calculation of inter-animal distances. These methods were developed and refined by Mark Johnson and Frants Jensen (for more details on both methods see Johnson, et al., 2015). Both of these methods rely on simultaneously tagging pairs of dolphins as well as identifying any other dolphins in the area whose calls may be confounding focal identification.

In terms of calls, this chapter focuses on echolocation clicks and signature whistles. In terms of the identification of clicks, they are highly directional, so comparing received amplitude between the two tags is not a reliable clue for discriminating the focal animal. However, since low frequency energy is less directional than the high frequency energy and sound production may produce low frequency vibrations in tissue to which the tag is attached, its presence indicates that this tagged animal is producing the clicks. Whistles are less directional, so I can rely more on the relative balance of amplitude as well as the angle of arrival of the sound to the tag: assuming the tag has not moved, that angle of arrival should be relatively stable for calls produced by the focal animal (Johnson et al., 2015). In order to take into account possible effects of the capture-release, I did not consider whistles that occurred during the first 30min post-tagging. Signature whistles were classified through spectrogram comparisons with animals' known signature whistles. Signature whistles with multiple loops that were separated by less than $250 \mathrm{~ms}$ 
were counted as a single whistle starting at the beginning of the first loop (Sayigh et al., 2007; H. Carter Esch et al., 2009; King et al., 2013).

To estimate a continuous measure of inter-animal distance I relied on the echolocation clicks. Once clicks recorded on each tagged dolphin (called focal clicks) have been identified, I can use clicks from each animal that are close together in time and heard on both tags in order to continuously estimate clock drift between the two simultaneous tags and inter-animal distance. This distance has a minimum standard deviation of the error of approximately $0.6 \mathrm{~m}$. I can then interpolate between these distance estimates using a Kalman filter. Since this method relies on hearing clicks, distance estimates are not available for long periods of silence. For a description of this method see Johnson et al. (2015).

I then used the distance calculations to identify separations and reunions for this study. A separation-reunion event was defined as a separation of greater than $30 \mathrm{~m}$. A common method for establishing criteria used to divide behaviors into categories is to identify a point of rapid change in a log-survival plot by finding the intersection between two regression lines for the pre and post-change slope values (Slater \& Lester, 1982). Employing this strategy with respect to inter-animal distance across all datasets showed a low inflection point at $7 \mathrm{~m}$ (Figure 3.1). I used this point as the minimum separation criterion and defined the entire separation-reunion event from $7 \mathrm{~m}$ out to its peak past $30 \mathrm{~m}$ and back to $7 \mathrm{~m}$. Only separations with a complete separation and reunion were included. 


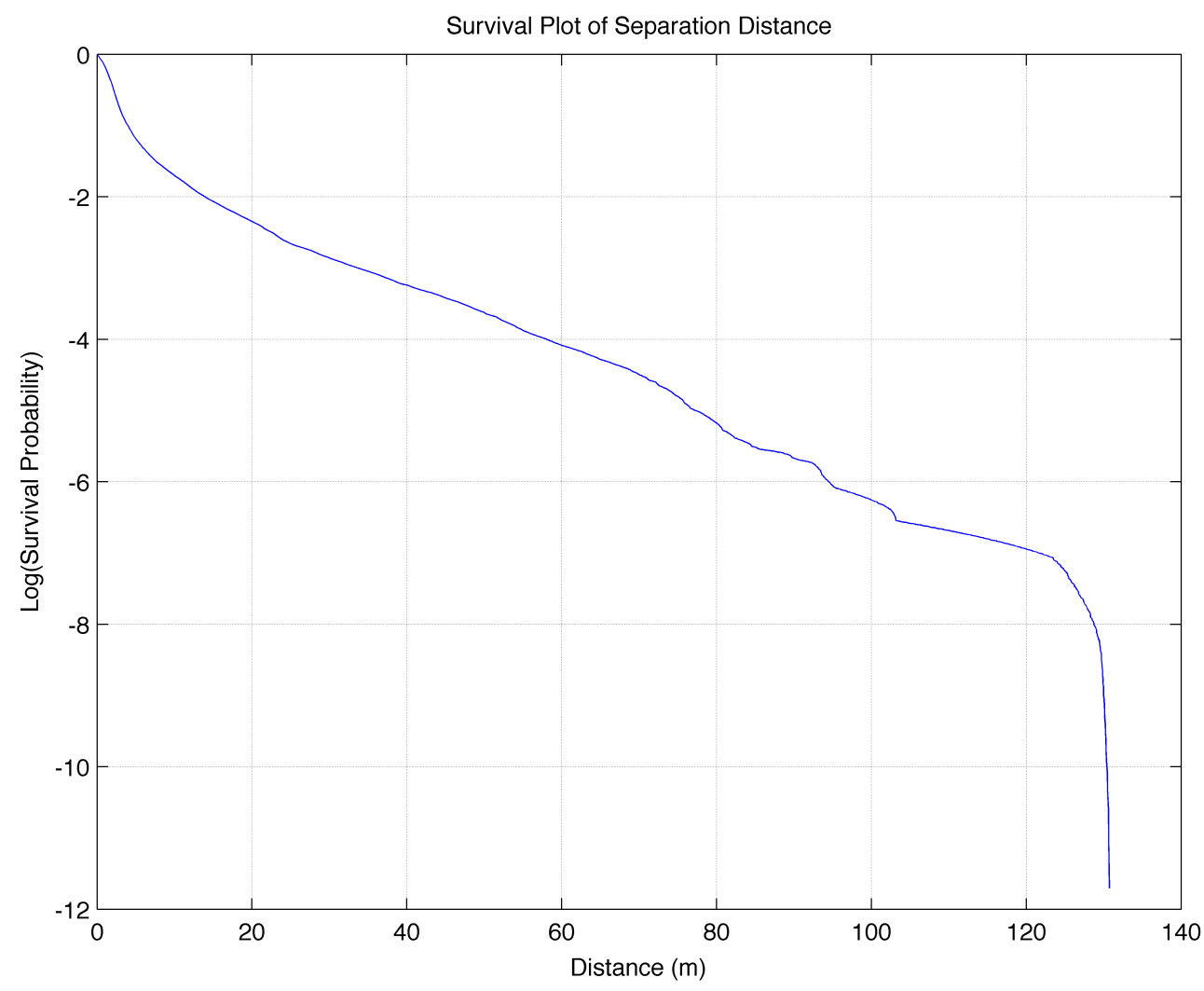

Figure 3.1: Log-survival plot of probability of inter-animal separation distance, pooled across $32 \mathrm{~h}$ of simultaneous tag deployments and 4 mother and calf pairs. A rapid change in slope occurs at $7 \mathrm{~m}$ of separation.

Figure 3.2 illustrates the relationship between animal tracks, separation distance, and the partnered dolphins spatial relationship over a 168s separation and reunion of one mothercalf pair. Tracks were reconstructed according to the method developed by Frants Jensen and described in Chapter Four. Signature whistles, super-imposed on the tracks appear to concentrate shortly after maximum separation as a reunion begins. 

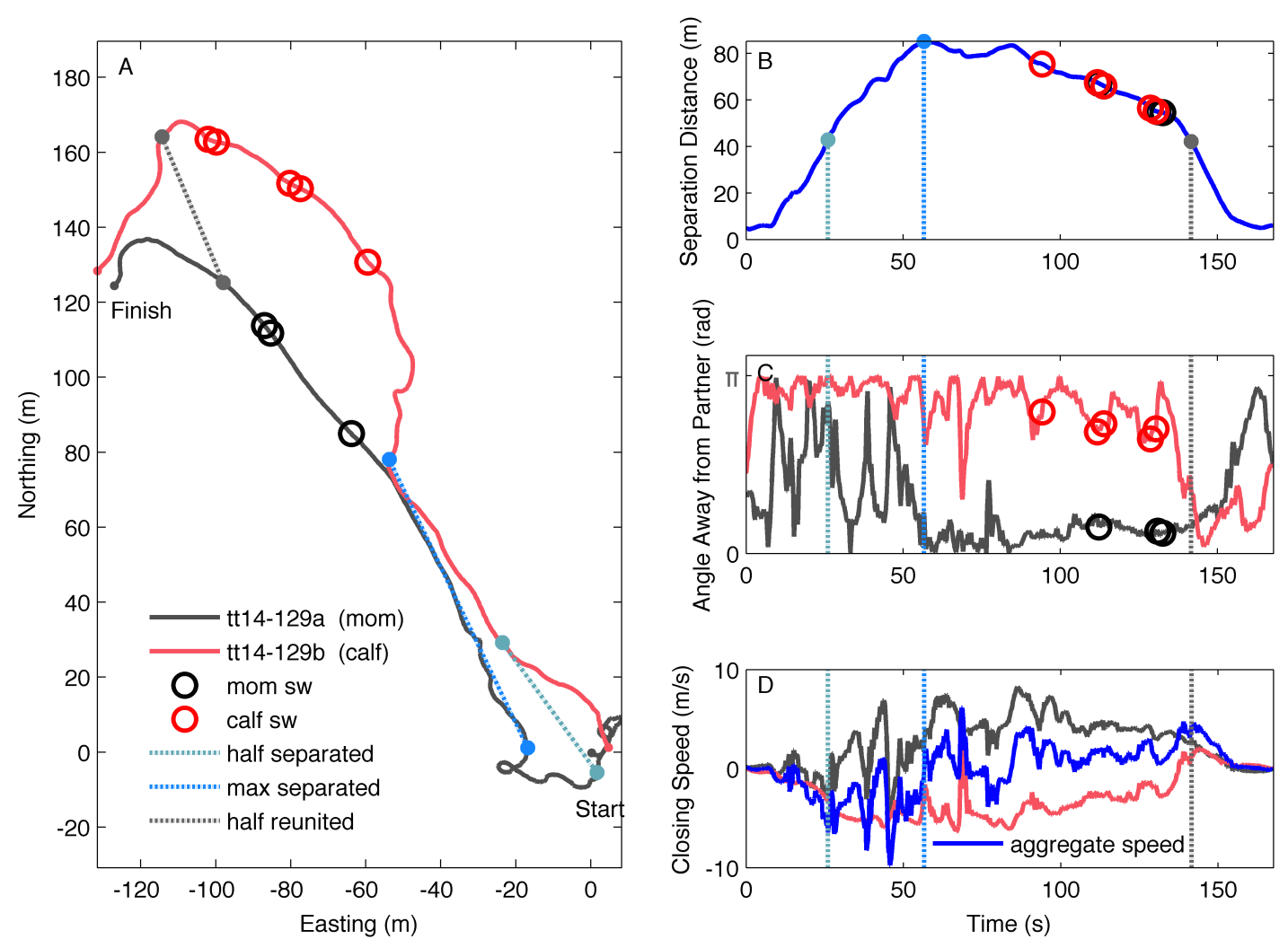

Figure 3.2: Example separation and reunion for a mother (tt14_129a, black) and her calf (tt14_129b, red). A. shows a 2D plan view of the animal tracks beginning at $(0,0)$. B. translates the track to a separationdistance timeseries between the dolphins. C. shows the absolute value of the heading angle in radians for each animal with respect to its partner with 0 radians representing the animal pointing directly at its partner. D. represents the speed with which an animal is closing the distance towards its partner. On all plots the time points representing a separation distance of halfway to maximum separation (teal), maximum separation (blue) and halfway to reunion (black) are marked as dotted lines. Signature whistles for each animal are shown as red and black circles.

\subsubsection{Model Fitting}

I then fit a logistic regression model of signature whistle call rate throughout separation and reunion events as a function of the square of percent separation distance, and separation phase. All model fitting was conducted using R (R Development Core Team, 2014). A plot of autocorrelation in the residuals for the final fitted model (Figure 
3.3) showed that the magnitude of correlation observed is small and would be unlikely to affect the results of the analysis. Since I have no behavioral reason to expect autocorrelation at the lags that show it, I treated the data as independent.

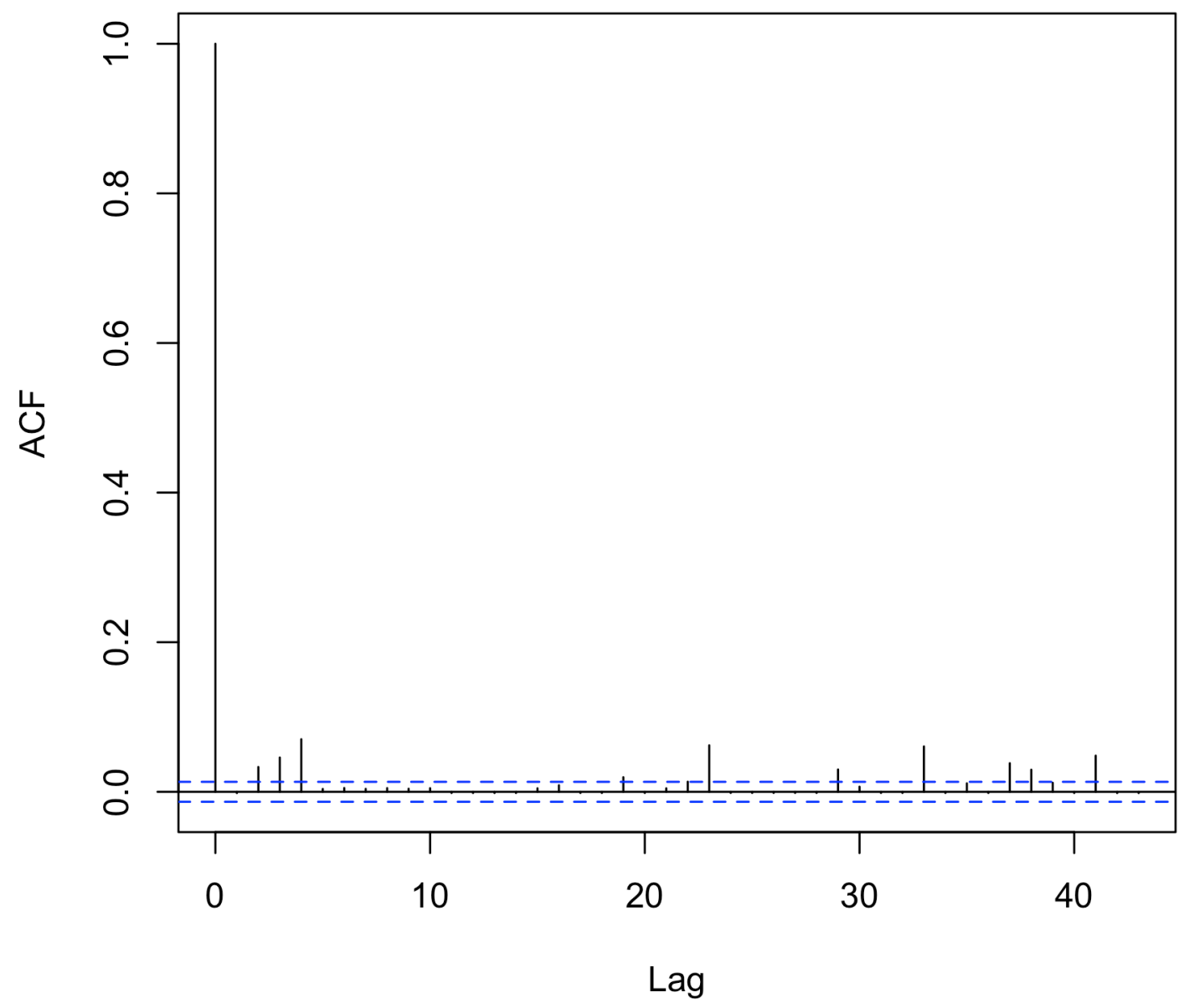

Figure 3.3: Autocorrelation function of the residuals for the final model, demonstrating that any evidence for correlation is small. Dotted blue lines represent 5\% significance level.

I began with a full complement of variables selected for biological relevance and chose the model using standard variable selection approaches at a significance threshold of $p=$ 0.05. The model fitting and selection process are described in more detail in the results. 


\subsection{RESULTS}

\subsubsection{Description of Dataset}

For this study, I focused on simultaneously tagged pairs of mothers and their calves. Over the last four field seasons, DTAGV3s have been simultaneously deployed on nine pairs of mothers and calves ranging in age from 11-43 and 2-7 years old respectively (Table 3.1). Five pairs had insufficient simultaneous tagout time or were lacking sufficient echolocation clicks to achieve accurate distance estimates and were excluded from the analysis. This left four pairs: one from 2013 and three from 2014 (pairs used for this analysis are shaded gray). One calf was male and the other three were female. Total simultaneous tag time for the analysis was $32.02 \mathrm{~h}$ spanning 25 separation and reunion events. 


\begin{tabular}{|c|c|c|c|c|c|}
\hline Field Season & Animal ID & Sex & Age & Tagout (h) & Separations \\
\hline \multirow{2}{*}{2011} & FB90 & Female & 41 & \multirow{2}{*}{0.82} & \multirow{2}{*}{ NA } \\
\hline & FB246 & Male & 7 & & \\
\hline \multirow{6}{*}{2012} & FB113 & Female & 16 & \multirow{2}{*}{0.63} & \multirow{2}{*}{ NA } \\
\hline & FB274 & Male & 2 & & \\
\hline & FB159 & Female & 16 & \multirow{2}{*}{0.96} & \multirow{2}{*}{ NA } \\
\hline & FB233 & Female & 2 & & \\
\hline & FB007 & Female & 28 & \multirow{2}{*}{2.8} & \multirow{2}{*}{ NA } \\
\hline & FB278 & Male & 2 & & \\
\hline \multirow{4}{*}{2013} & FB90 & Female & 43 & \multirow{2}{*}{0.72} & \multirow{2}{*}{ NA } \\
\hline & FB270 & Male & 4 & & \\
\hline & FB33 & Female & 31 & \multirow{2}{*}{2.91} & \multirow{2}{*}{5} \\
\hline & FB282 & Male & 5 & & \\
\hline \multirow{6}{*}{2014} & FB197 & Female & 11 & \multirow{2}{*}{18.18} & \multirow{2}{*}{6} \\
\hline & FB243 & Female & 2 & & \\
\hline & FB133 & Female & 15 & \multirow{2}{*}{6.18} & \multirow{2}{*}{3} \\
\hline & FB245 & Female & 2 & & \\
\hline & FB185 & Female & 14 & \multirow{2}{*}{4.75} & \multirow{2}{*}{11} \\
\hline & FB249 & Female & 3 & & \\
\hline
\end{tabular}

Table 3.1: Summary of simultaneous tag deployments on 9 mother and calf pairs across the last 4 field seasons. Pairs included in the analysis are shaded gray.

Inter-animal distances were calculated for these four mother-calf pairs. Every instance of a tagged animal's signature whistle was also identified from the acoustic record. These signature whistles are overlaid as circles on the plots of inter-animal distance shown in Figure 3.4A-D. Those produced by the mothers are shown in magenta and calves in black. During instances with no echolocation clicks close together in time it is not possible to estimate inter-animal distance, so no data are shown. 

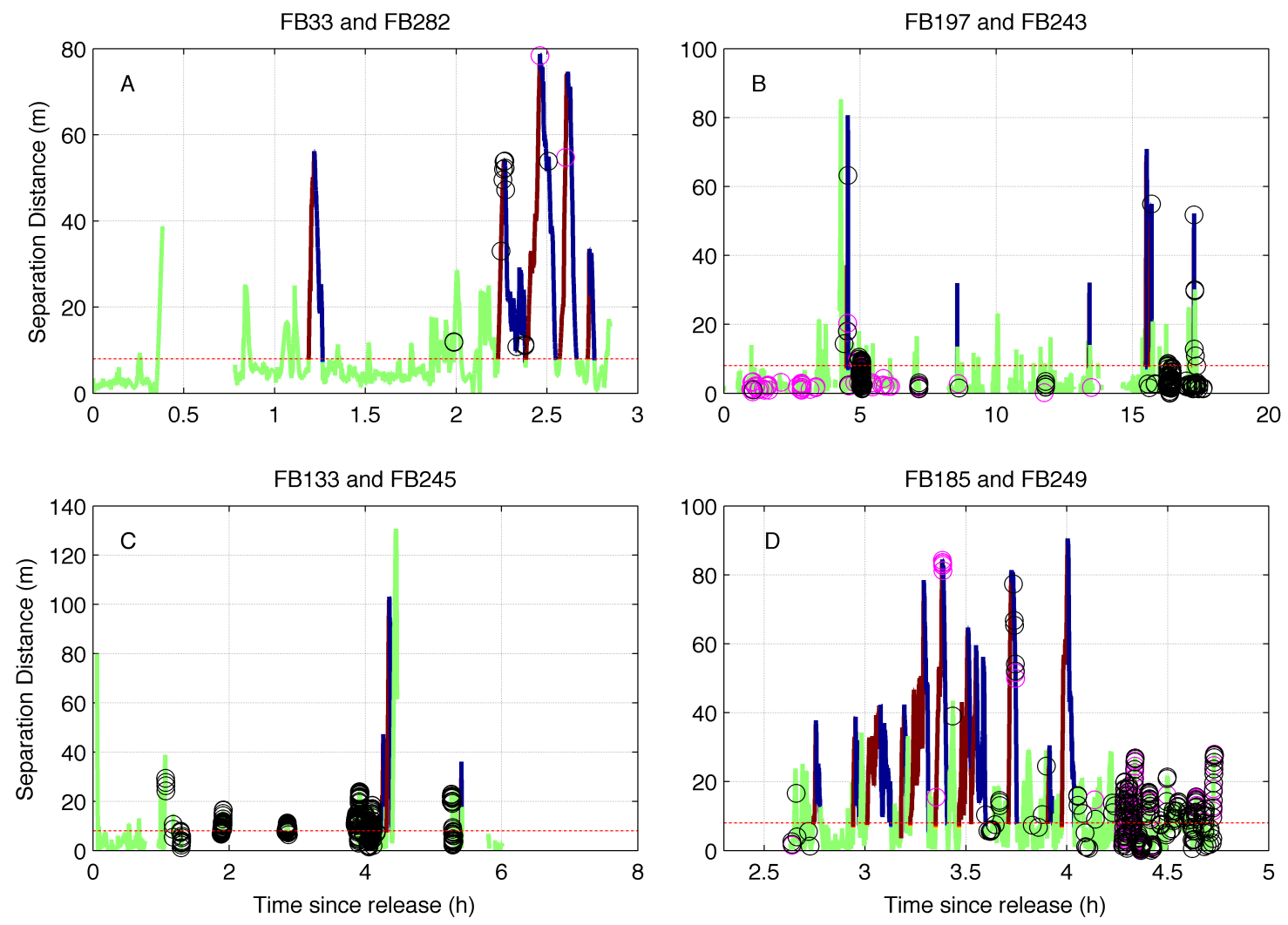

Figure 3.4: Simultaneous deployments on 4 mother-calf pairs: FB33 and FB282 (A, 2.82h, 4 complete separations); FB197 and FB243 (B, 18.18h, 6 complete separations); FB133 and FB245(C, 6.18h, 3 complete separations); FB185 and FB249 (D, 4.75h, 11 complete separations) Separations are shown in dark red, reunions in blue and other times in green. Times with insufficient data to estimate distance are left blank. Signature whistles for the mother (magenta) and calf (black) are shown as circles placed at the beginning of each whistle. Notice that the $\mathrm{x}$-axis for $\mathrm{D}$ is shifted to zoom in on the period when separations and reunions were observed.

Table 3.2 shows aggregated counts of signature whistles, separated by animal and by phase in a separation event. In general, calves are more vocal than mothers, and both mothers and calves produce more signature whistles when they are not in a separationreunion event (close) than they do when splitting apart greater than $30 \mathrm{~m}$ (separation or reunion). However, these are total counts not normalized for time, and that observation 
needs to be interpreted within the context of them spending a much smaller proportion of the tagout time in separation-reunion events than they do close together.

Signature Whistle Counts by Phase

\begin{tabular}{l|r|r|r|r}
\hline \hline ID & close & separation & reunion & total \\
\hline FB33 & 0 & 2 & 0 & 2 \\
FB282 & 2 & 4 & 7 & 13 \\
FB197 & 100 & 2 & 0 & 102 \\
FB243 & 317 & 1 & 4 & 322 \\
FB133 & 8 & 0 & 0 & 8 \\
FB245 & 738 & 0 & 0 & 738 \\
FB185 & 114 & 1 & 8 & 123 \\
FB249 & 217 & 0 & 6 & 223 \\
\hline all mothers & 222 & 5 & 8 & $\mathbf{2 3 5}$ \\
all calves & 1274 & 5 & 17 & $\mathbf{1 2 9 6}$ \\
\hline \hline total & 1496 & 10 & 25 & $\mathbf{1 5 3 1}$
\end{tabular}

Table 3.2: Counts of signature whistles produced by each tagged dolphin throughout the entire set of simultaneous tag deployments. Counts are separated both by animal, age class and phase. Close represents all times when animals are not in a separation-reunion event as defined in the methods. Mothers are shaded gray.

\subsubsection{Data for Model Fitting:}

To fit the model, I only considered time during complete separation and reunion events that have data for the entire separation and reunion. Every 0.5 seconds in those separation-reunion events I calculated a measure of separation distance as the percent distance out to the maximum separation in that particular event. For example, if two dolphins begin at a separation of $7 \mathrm{~m}$, go out to a maximum of $50 \mathrm{~m}$ and are at $15 \mathrm{~m}$ on their return to $7 \mathrm{~m}$, they would have moved from a percent distance value of $14 \%$ out to $100 \%$ and be back to $30 \%$. On the other hand, if they had started at $7 \mathrm{~m}$ but separated to $100 \mathrm{~m}$ and are at $15 \mathrm{~m}$ on their return to $7 \mathrm{~m}$, they would have moved from $7 \%$ out to 
$100 \%$ and back to $15 \%$. I chose percent distance rather than absolute distance to be able to compare across separation and reunion events with different maximum separation distances. Along with percent distance, each time point also recorded whether the animal was in a separation or reunion phase, animal identity, age class (mom or calf), whether or not the animal was in a phase of the separation between 10s prior to peak and 50s after maximum separation.

\subsubsection{Variable Selection Process and Results}

I began model selection by plotting signature whistle counts at binned percent distance separation ranges. In the resulting histogram (Figure 3.5), frequencies tended to be higher when distances were very low or very high, suggesting a quadratic model of percent distance as a basis for the logistic regression. 


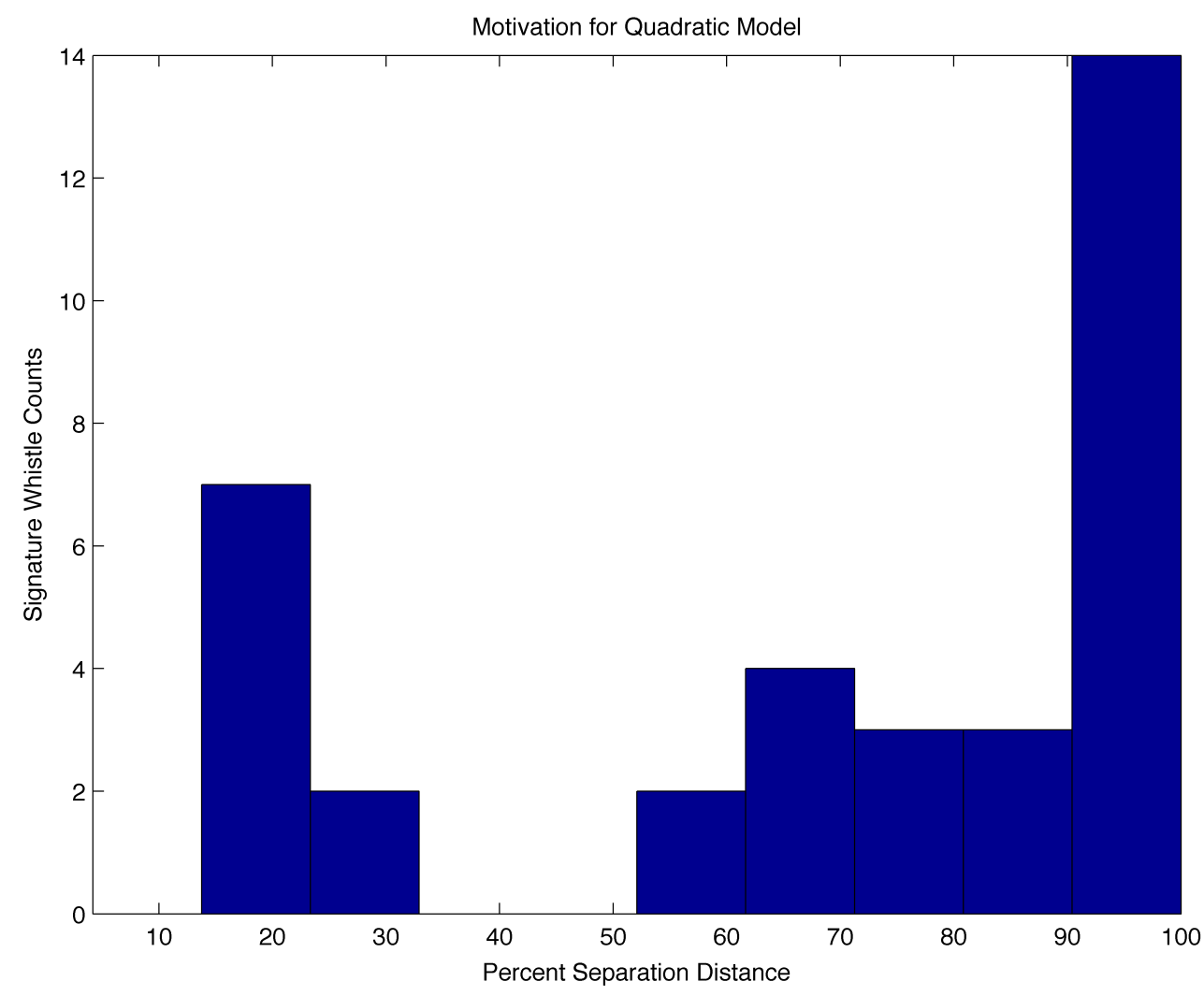

Figure 3.5. Signature whistle counts binned according to percent separation distance, suggesting a parametric quadratic regression as the basis for the model.

As described in the methods, I fitted a quadratic logistic regression model. The model used a binomial family with a logit link function. The first model fit the probability of an animal producing a signature whistle during a separation and reunionevent as a function of the following variables: the square of percent distance in a separation (i.e. Peak separation is $100 \%$ ), percent distance, whether an animal was in a separation or reunion phase, whether the animal was a mother or calf, whether or not the animal was in a phase of the separation between 10s prior to peak and 50s after maximum separation and animal identity. Backwards stepwise variable selection at threshold of $\mathrm{p}=$ 0.05 left a model of probability of signature whistle during a separation and reunion event 
as a function of the square of percent distance, percent distance and whether or not the animal was in a separation or reunion.

$$
\log \left(\frac{p(s w)}{1-p(s w)}\right)=\beta_{0}+\beta_{1} \% \text { Dist }{ }^{2}+\beta_{2} \% \text { Dist }+\beta_{3} \text { Separation }
$$

Table 3.3 reports model coefficients and p-values.

\begin{tabular}{r|rrrr} 
sw & Coef. & Std. Err. & $\mathbf{z}$ & $\mathbf{p}$ \\
\hline Intercept & -4.6816626 & 0.8012930 & -5.843 & $5.14 \mathrm{e}-09$ \\
Percent Distance $^{2}$ & 0.0009654 & 0.0002744 & 3.518 & 0.000435 \\
Percent Distance & -0.0962486 & 0.0329545 & -2.921 & 0.003493 \\
In Separation & -0.8205444 & 0.3751565 & -2.187 & 0.028728 \\
\hline
\end{tabular}

Table 3.3. Summary of the binomial logistic regression model showing that the probability of a signature whistle was best explained by percent distance and whether or not an animal was in a separation or a reunion

Figure 3.6 plots the fitted probability of a signature whistle as a function of percent distance in a separation and reunion event divided into the separation and reunion phases. 


\section{Separations}

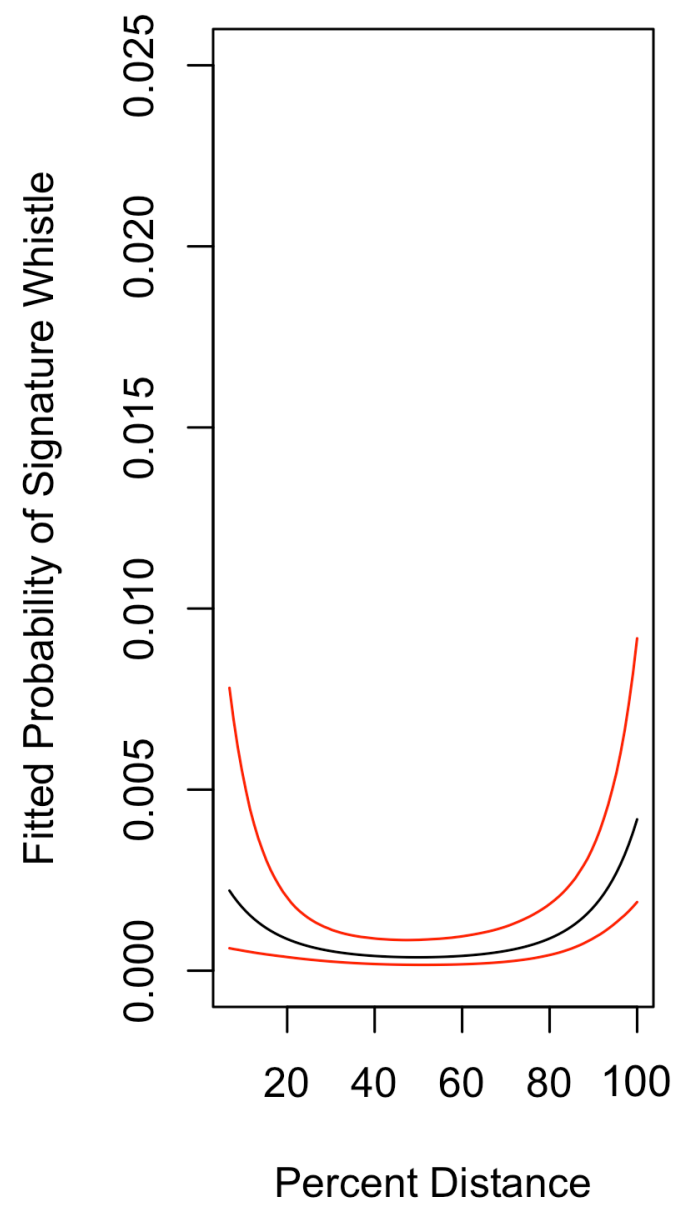

Reunions

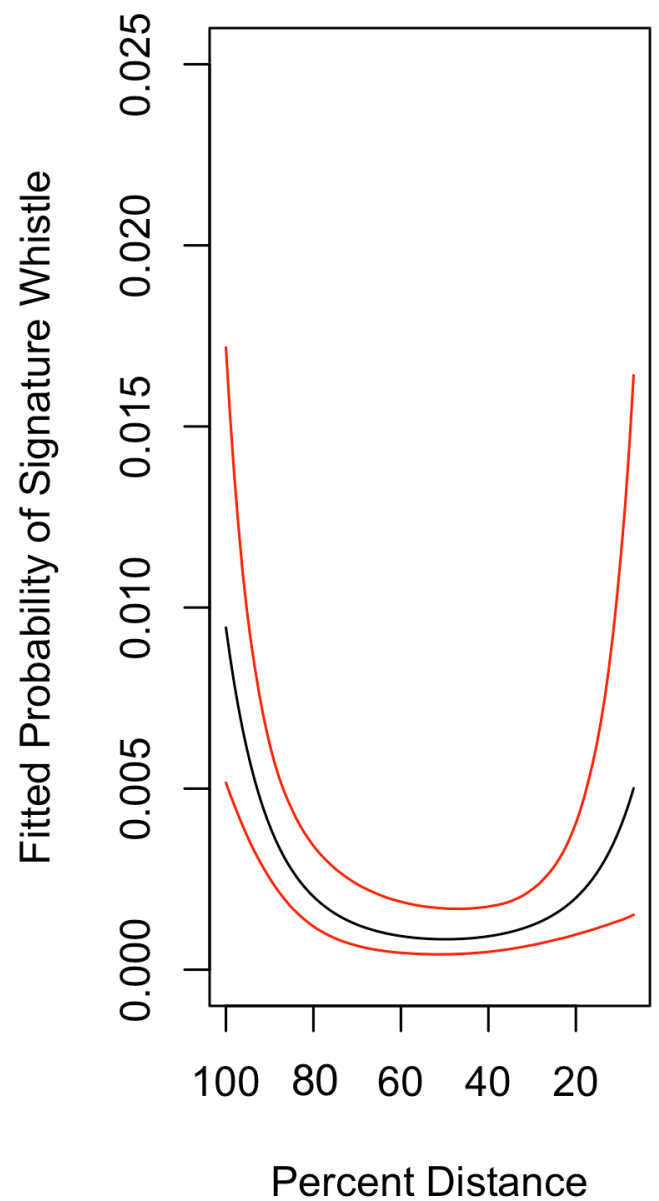

Figure 3.6: Logistic regression model of the probability of a signature whistle during a separation-and-reunion event as a function of percent distance and whether or not the animal was in a separation or reunion. $95 \%$ confidence intervals are shown in red. Separations and reunions are shown separately. Note that the $\mathrm{x}$-axis for reunions begins at 100 percent separation, so that the two plots mimic the overall shape of an event.

While the confidence intervals suggest that more data would be useful, I can cautiously interpret a few things about signature whistle production during separation and reunion events: there seems to be a difference between separations and reunions with a trend 
towards higher probability of signature whistles during reunions. There also appears to be a trend towards more whistles as inter-animal distance increases.

\subsection{DISCUSSION}

In order to interpret these data I will discuss the unexpected, but quite interesting finding that the vast majority of signature whistles do not occur during separations and reunions. Then I will consider each hypothesis in turn and outline some exciting opportunities for next steps before discussing differences between the Sarasota field site and the open ocean.

First of all, when looking at the distribution of whistles across the deployments, $97 \%$ of signature whistles occur when the animals are close together, rather than when they are in a separation-reunion event. These large numbers of high amplitude and increasingly noisy signature whistles are clustered in tight bouts when animals are close together. Figure 3.7 shows an excerpt from the middle of one of these bouts produced by a calf, FB243. 

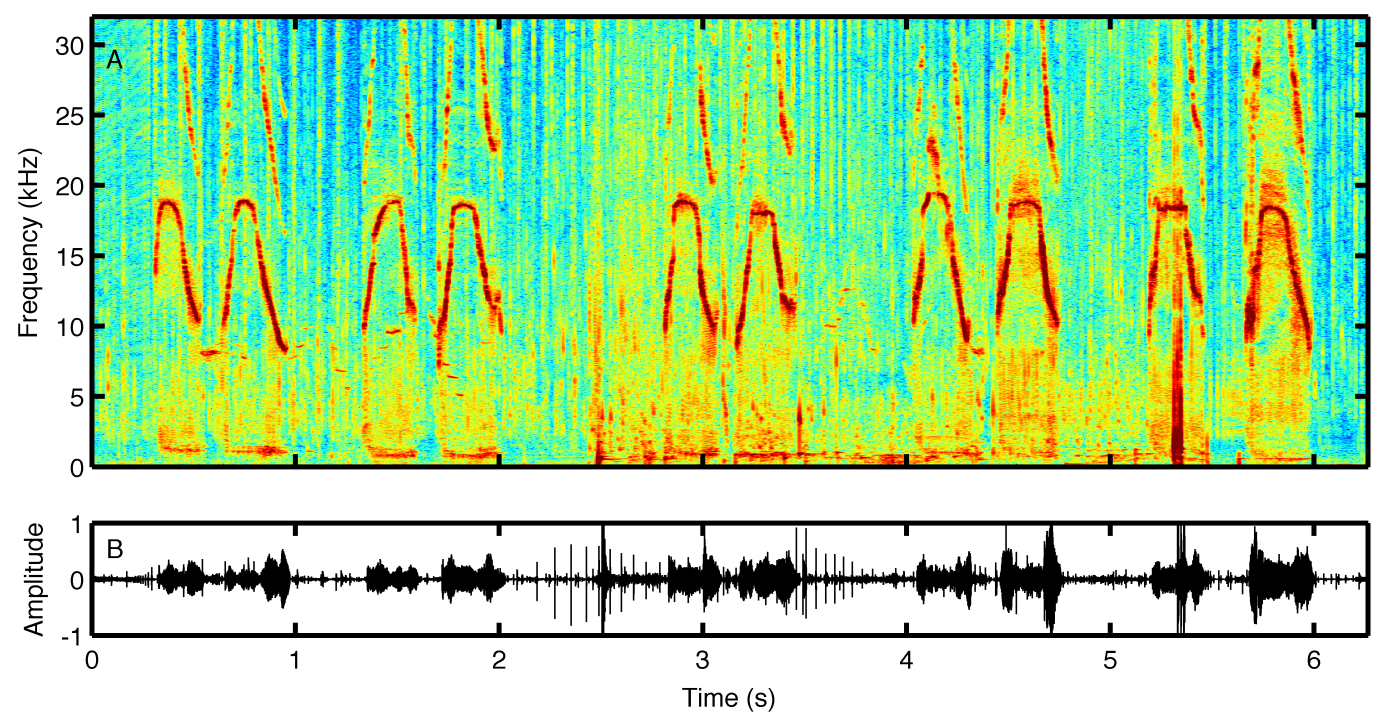

Figure 3.7: Excerpt from a tight bout of highly repeated signature whistles produced by FB243 when the animals were less than $7 \mathrm{~m}$ apart. FFT size 8192, Hanning window, temporal overlap of $90 \%$.

While this pattern of high rates of whistling in tightly clustered bouts when animals are close holds for the pairs shown in Figure 3.4B-D, it was not observed in Figure 3.4A. It was predominantly observed in calves rather than their mothers. Calves also have higher signature whistle rates than mothers, producing 1296 signature whistles over all the deployments as opposed to 235 . As outlined in the introduction, signature whistles are often associated with stress and other internal states of animals (Lilly, 1963; H. C. Esch et al., 2009). It is likely that signature whistles may be serving a continuum of functions that differ when animals are close together rather than separating and reuniting.

In mammals, this kind of intense infant calling is often related to parent-offspring conflict when the offspring is requesting care that the parent is denying. These infant care elicitation calls are observed in a variety of primates such as vervet monkeys (Hauser, 1993), baboons (Barrett \& Henzi, 2000), and rhesus macaques (Berman et al., 1993). Suckling is one of the best-studied cases: as the mother rejects suckling, the calls increase 
and become more and more intense (Maestripieri, 2002). Bottlenose dolphins have an extended lactation period of several years (Wells, 1991), and one instance where intense signature whistle bouts like these were observed when a calf wished to nurse and the mother rejected it (Janik, VM P. Comm, Nov 17, 2014). While I did observe some nursing behavior, the intense bouts did not overlap with the focal follows, so behavioral observations cannot provide context. High rates of signature whistle production also occur when a calf encounters another calf (Janik, 2014), so it is possible that a similar situation accounts for some of the high rates. Without behavioral observations it is impossible to know for certain what was occurring during these bouts; however, there does appear to be a distinction in functional modes between the intense bouts and the much lower rates when animals are separating and reuniting.

Returning to these separation-reunion events, I began with three hypotheses: that signature whistles in separations and reunions might serve location monitoring, reunion contact call or identity confirmation functions.

Beginning with the location monitoring hypothesis, I expected that evidence for this function would be a steady and random signature whistle rate above a certain distance but with no trend for approach immediately after whistling. The data do not seem to support this conclusion because of the observed differences in signature whistle rates between separations and reunions. Dolphins appear to produce more signature whistles during reunions than separations (25 vs 10), and the difference between separations and reunions was a significant factor in the model. Despite the broad confidence intervals, Figure 3.6 also shows differences in shapes between the separation and reunion curves, which is inconsistent with a hypothesis of random distribution of 
signature whistles. One potential confound to consider is that during the separation phase of a separation-event, dolphins may have a clearer sense of their partner's location, for less time has elapsed since they were together, reducing the need for location monitoring. This might lead to a lower rate during separations than reunions. Although this is impossible to rule out, the fact that the separation to peak distance lasted a range of different times yet still showed a significant difference between separations and reunions suggests that these data are not consistent with location monitoring.

With respect to a potential reunion call function, I expected that signature whistles representing a motivation to reunite would be concentrated near the maximum point of separation, prior to animals coming back together. Several separation-reunion events throughout the deployments do show clusters coinciding at the peaks (e.g. separations 5 and 6 in Figure 3.4B). Moreover, there does appear to be a general trend towards increased whistle rates as the distances between the animals increase in a separationreunion event. The model fitting in Figure 3.6 is consistent with this hypothesis and shows a higher probability of producing a signature whistle when animals are close to peak separation, with there also being a slight skewing towards a concentration at the beginning of reunions. These results provide evidence supporting the reunion hypothesis, but there are insufficient data to make any very strong claims, so interpretations must be cautious.

In terms of the third identity confirmation function, I would expect supporting data to show another concentration of signature whistles just prior to the end of the reunion. This function would be in line with previous studies reporting identity information in signature whistles and instances of animals exchanging them at sea when 
encountering another group (Quick \& Janik, 2012). I did observe examples of signature whistles occurring just as animals were finishing a reunion (e.g. separation 2 in Figure 3.4A). Figure 3.6 also shows a potential for an increased probability of signature whistles at that time. However, with such a small number of whistles and such wide confidence bounds at the edges, more data are needed to truly assess that hypothesis.

Given these pilot results that are consistent with a reunion call function role for signature whistles, one fruitful way to further explore their function in wild dolphins might be to use playback experiments. During a follow of a dolphin pair, we could wait for natural separations, position the follow vessel between the animals and play one animal's signature whistle to its partner and observe the reaction. If signature whistles are used to represent a motivation to reunite when in a separation, I might expect the receiving animal to orient itself to the animal whose signature whistle was played and perhaps begin to move in that direction. Although the model did not show a significant difference between mothers and calves, I did observe a higher calf vocal rate, and the lack of difference might have been impeded by low statistical power due to small sample size. It would be particularly interesting to contrast the difference between a mother and calf in terms of their reactions to the other animal's signature whistle. This could give us a sense of the animal responsible for separations and reunions that is not apparent in the absolute distance calculations.

This chapter has focused on the first context where it has been possible to tag wild bottlenose dolphins. However, many dolphins live in other environments, and there are some considerations for extrapolating these results to oceanic contexts. The dolphins in Sarasota live primarily in shallow inter-coastal waters with brief excursions into Tampa 
Bay or the Gulf of Mexico. This means that they essentially inhabit a two-dimensional environment filled with inland channels and obstacles that they may be able to use for shelter. In contrast, the open ocean is a truly three-dimensional environment with little refuge, which may influence their vocal and spatial behavior. The techniques developed here to identify spacing and the vocalizing individual could be equally applied to oceanic environments. However, the third dimension would need to be taken into account, for example in the creation of the animal tracks (Figure 3.2). In the open ocean, lone animals would be even more exposed, and with less shelter defending the safety of the group might be more essential for survival than in Sarasota. Under these circumstances, I would expect cohesion to be even more important that in the inter-coastal waters, and the mechanisms for mediating cohesion might emerge in even stronger ways than in Sarasota. 


\subsection{REFERENCES:}

Aubin, T., \& Jouventin, P. (2002). How to vocally identify kin in a crowd: the penguin model. Advances in the Study of Behavior, 31, 243-277.

Barrett, L., \& Henzi, S. P. (2000). Are baboon infants Sir Phillip Sydney's offspring? Ethology, 106(7), 645-658.

Berman, C., Rasmussen, K., \& Suomi, S. (1993). Reproductive consequences of maternal care patterns during estrus among free-ranging rhesus monkeys. Behavioral Ecology and Sociobiology, 32(6), 391-399.

Bradbury, J. W., \& Vehrencamp, S. L. (1998). Principles of animal communication.

Caine, N. G., \& Stevens, C. (1990). Evidence for a oductive consequences of-bellied tamarins. American Journal of Primatology, 22(4), 251-262.

Caldwell, M. C., \& Caldwell, D. K. (1965). Individualized Whistle Contours in BottleNosed Dolphins (Tursiops Truncatus). Nature, 207(4995), 434-\&.

Caldwell, M. C., Caldwell, D. K., \& Tyack, P. L. (1990). Review of the signature-whistle hypothesis for the Atlantic bottlenose dolphin. In S. Leatherwood \& R. R. Reeves (Eds.), The Bottlenose Dolphin (pp. 199-234): New York: Academic Press.

Connor, R. C., Smolker, R. A., \& Richards, A. F. (1992). Two levels of alliance formation among male bottlenose dolphins (Tursiops Sp). Proceedings of the National Academy of Sciences, 89(3), 987-990.

Connor, R. C., Wells, R. S., Mann, J., \& Read, A. J. (2000). The Bottlenose Dolphin. In J. Mann, R. C. Connor, P. L. Tyack \& H. Whitehead (Eds.), Cetacean Societies: Field studies of whales and dolphins (pp. 91-126). Chicago: Univ. Chicago Press.

Da Cunha, R. G. T., \& Byrne, R. W. (2009). The use of vocal communication in keeping the spatial cohesion of groups: Intentionality and specific functions. In P. A. Garber, A. Estrada, J. C. Bicca-Marques, E. W. Heymann \& K. B. Strier (Eds.), South American Primates (pp. 341-363): Springer New York.

Esch, H. C., Sayigh, L. S., Blum, J. E., \& Wells, R. S. (2009). Whistles as potential indicators of stress in bottlenose dolphins (Tursiops truncatus). Journal of Mammalogy, 90(3), 638-650.

Esch, H. C., Sayigh, L. S., \& Wells, R. S. (2009). Quantifying parameters of bottlenose dolphin signature whistles. Marine Mammal Science, 25(4), 976-986. doi: 10.1111/j.1748-7692.2009.00289.x

Hauser, M. (1993). Do vervet monkey infants cry wolf? Animal Behaviour, 45(6), 12421244.

Janik, V. M. (2009). Acoustic communication in delphinids. In M. Naguib, V. Janik, N. Clayton \& K. Zuberbuhler (Eds.), Advances in the Study of Behavior (Vol. 40, pp. 123-157): Academic Press.

Janik, V. M. (2014, Nov 17, 2014).

Janik, V. M., \& Sayigh, L. S. (2013). Communication in bottlenose dolphins: 50 years of signature whistle research. Journal of Comparative Physiology A, 199(6), 479489.

Janik, V. M., \& Slater, P. J. (1998). Context-specific use suggests that bottlenose dolphin signature whistles are cohesion calls. Animal Behaviour, 56, 829-838. 
Johnson, M., de Soto, N. A., \& Madsen, P. T. (2009). Studying the behaviour and sensory ecology of marine mammals using acoustic recording tags: a review. Marine Ecology Progress Series, 395, 55-73. doi: 10.3354/meps08255

Johnson, M., Jensen, F. H., Miller, P., Madsen, P. T., de Soto, N. A., \& Tyack, P. L. (2015). Measuring the separation distance and relative location of animals using sound recording tags. Behavioral Ecology and Sociobiology, Submitted.

Johnson, M. P., \& Tyack, P. L. (2003). A digital acoustic recording tag for measuring the response of wild marine mammals to sound. IEEE Journal of Oceanic Engineering, 28(1), 3-12.

King, S. L., Sayigh, L. S., Wells, R. S., Fellner, W., \& Janik, V. M. (2013). Vocal copying of individually distinctive signature whistles in bottlenose dolphins. Proceedings of the Royal Society B: Biological Sciences, 280(1757).

Kondo, N., \& Watanabe, S. (2009). Contact calls: information and social function. Japanese Psychological Research, 51(3), 197-208.

Lilly, J. C. (1963). Distress call of the bottlenose dolphin: stimuli and evoked behavioral responses. Science, 139(3550), 116-118.

Maestripieri, D. (2002). Parent-offspring conflict in primates. International Journal of Primatology, 23(4), 923-951.

Newman, J. D. (1985). The infant cry of primates Infant crying (pp. 307-323): Springer.

Owen, E. C., Wells, R. S., \& Hofmann, S. (2002). Ranging and association patterns of paired and unpaired adult male Atlantic bottlenose dolphins, Tursiops truncatus, in Sarasota, Florida, provide no evidence for alternative male strategies. Canadian Journal of Zoology, 80(12), 2072-2089.

Quick, N. J., \& Janik, V. M. (2012). Bottlenose dolphins exchange signature whistles when meeting at sea. Proceedings of the Royal Society B: Biological Sciences, 279, 2539-2545.

R Development Core Team. (2014). R: A language and environment for statistical computing. Vienna, Austria: R Foundation for Statistical Computing.

Ramos-Fernández, G. (2005). Vocal communication in a fission-fusion society: do spider monkeys stay in touch with close associates? International Journal of Primatology, 26(5), 1077-1092.

Sayigh, L. S., Esch, H. C., Wells, R. S., \& Janik, V. M. (2007). Facts about signature whistles of bottlenose dolphins, Tursiops truncatus. Animal Behaviour, 74(6), 1631-1642.

Scott, M., Wells, R., \& Irvine, A. B. (1990). A long-term study of bottlenose dolphins on the West coast of Florida. In S. Leatherwood \& R. R. Reeves (Eds.), The bottlenose dolphin (pp. 235-244). San Diego: Academic Press.

Searby, A., Jouventin, P., \& Aubin, T. (2004). Acoustic recognition in macaroni penguins: an original signature system. Animal Behaviour, 67(4), 615-625.

Slater, P., \& Lester, N. (1982). Minimising errors in splitting behaviour into bouts. Behaviour, 153-161.

Smolker, R., Mann, J., \& Smuts, B. (1993). Use of signature whistles during separations and reunions by wild bottlenose dolphin mothers and infants. Behavioral Ecology and Sociobiology, 33(6), 393-402. 
Townsend, S. W., Hollén, L. I., \& Manser, M. B. (2010). Meerkat close calls encode group-specific signatures, but receivers fail to discriminate. Animal Behaviour, 80(1), 133-138.

Vehrencamp, S. L., Ritter, A., Keever, M., \& Bradbury, J. (2003). Responses to Playback of Local vs. Distant Contact Calls in the Orange-Fronted Conure, Aratinga canicularis. Ethology, 109(1), 37-54.

Watwood, S. L., Owen, E. C. G., Tyack, P. L., \& Wells, R. S. (2005). Signature whistle use by temporarily restrained and free-swimming bottlenose dolphins, Tursiops truncatus. Animal Behaviour, 69, 1373-1386.

Wells, R. S. (1991). The role of long-term study in understanding the social structure of a bottlenose dolphin community. In K. Pryor \& K. Norris (Eds.), Dolphin societies: discoveries and puzzles (pp. 199-225). Berkeley: University of California Press.

Wells, R. S. (2003). Dolphin social complexity: lessons from long-term study and lifehistory. In F. B. M. De Waal \& P. L. Tyack (Eds.), Animal social complexity: intelligence, culture, and individualized societies. Cambridge: Harvard University Press.

Wells, R. S. (2009). Learning from nature: bottlenose dolphin care and husbandry. Zoo Biology, 28(6), 635-651.

Wilkinson, G. S., \& Boughman, J. W. (1998). Social calls coordinate foraging in greater spear-nosed bats. Animal Behaviour, 55(2), 337-350. 


\section{Chapter 4: Do dolphins keep track of their partners by passively monitoring conspecific biosonar signals?}




\subsection{ABSTRaCT}

Social bottlenose dolphins live in fission fusion societies, forming transient associations while maintaining strong bonds with a few key individuals. They use individuallydistinctive signature whistles for recognition, contact and spatial coordination (Chapter Three). Like other odontocetes, they also gather information about their environment using echolocation, and these sonar clicks are audible to other animals. Here I test whether dolphins use these echolocation signals from conspecifics to keep track of partners when separated. This exploration takes advantage of the unique opportunity provided by simultaneously tagging pairs of dolphins in order to investigate sonar transmission in their natural habitat, allowing consideration of distributions of data from on-animal tags that are better reflections of dolphin behavior and transmission loss in the natural environment, rather than single measurements taken in captivity. The chapter begins by introducing the dataset and moving through an exploration of a dolphin's beam pattern by reconstructing animal trajectories across separations. Then three complementary approaches address the question of this strategy's potential range: 1) by modeling the distribution of maximum detected ranges from each click. 2) by separating clicks according to whether they are in the forward or aft portions of the beam patterns to create probability density functions of maximum detection ranges that a dolphin is likely to encounter. 3) by investigating the interaction of range with the probability of an emitted click being detected by a partner. This multi-pronged approach demonstrates that the operating range of this listening strategy overlaps with the typical separation ranges of Sarasota mother-calf pairs. Moreover, the model from Chapter Three can be significantly improved by adding a variable to capture the delay since the tag on an animal last detected a sonar click from its partner.

\subsection{INTRODUCTION}

Biologists typically maintain a rigid distinction between vocalizations used for communication and those used for echolocation. However, amongst social animals, this separation may be artificial. While echolocating on prey during foraging, an individual is not only actively exploring its environment but also broadcasting its own location-and even information about its current behavior and the presence of food - to any animal able to hear. As described in Chapter Three, coordinating spatial cohesion forms an essential part of animal communication, and if sonar clicks can provide useful information to conspecifics about the location of partners, perhaps they should not be excluded from the 
communication sphere. Cvikel et al. (2015) report a recent example in a foraging bat, Rhinopoma microphyllum: by combining GPS tags with ultrasonic recorders, they were able to show that bats passively eavesdrop on the echolocation clicks of conspecifics in order to obtain spatial information about patches of prey while foraging. Might a similar strategy be employed in a species like the bottlenose dolphin, Tursiops truncatus, to keep track of conspecifics?

As described in Chapters Two and Three, social bottlenose dolphins form transient associations with many animals in their fission-fusion societies, all the while maintaining strong, stable bonds with a few key individuals (Connor et al., 2000). With limited visual acuity and olfactory senses, dolphins must rely on acoustic signals to keep in touch with partner animals while separated (Janik, 2009). Individually-distinctive signature whistles appear to be one of these signals (Chapter Three); might echolocation clicks be another? Just as the bats eavesdrop on each other's clicks to detect the location of prey, dolphins could take advantage of a similar strategy to monitor their partners' locations. I will use the term echolocation monitoring to refer to the act of monitoring the location of an echolocating conspecific by passively listening to their sonar clicks. In this chapter, I will investigate the distances over which a dolphin might use such an echolocation monitoring strategy.

This approach takes advantage of the unique opportunity provided by simultaneously tagging pairs of dolphins in order to explore sonar transmission in their natural habitat. One of the strengths of this strategy is that it allows us to consider distributions rather than individual measurements, providing a more probabilistic 
characterization of transmission by wild dolphins whose behavior and environment are constantly changing.

This chapter begins by introducing current research on active space, followed by a description of the data used to address the distances over which a Sarasota dolphin could use an echolocation monitoring approach, and a preliminary comparison between the potential detection ranges of passive and active sonar. Afterwards, I move through an exploration of a dolphin beam pattern using simultaneous tag data in order to reconstruct animal trajectories across separations and calculate the off-axis angle of each click as heard by the tag on the other dolphin. Then, I approach the question of echolocation monitoring range from three complementary directions: 1) by modeling the distribution of maximum detection ranges from every detected click; 2) by separating clicks according to their beam pattern to create probability density functions of the maximum detection ranges that a dolphin is likely to encounter when pointing away from or towards its partner; and 3) by calculating the probability that an emitted click is detected based on separation range. Once I have an idea of the realistic detection ranges for naturally behaving dolphins, I use the model created in Chapter Three in order to explore how the idea of echolocation monitoring strategy might influence the signature whistle calling probability of spatially separated pairs of dolphins.

\subsection{ACTIVE SPACE}

The active space of a signal refers to the effective range over which it can be transmitted and then detected by a receiver. It is a function of the signal's source level $(S L)$, the transmission loss $(T L)$ that occurs as the signal travels, ambient noise levels 
$(N L)$, and the hearing capabilities of the receiver (Marten \& Marler, 1977). For a receiver to detect a signal, the signal must exceed the hearing threshold of the animal and be greater than the critical ratio above the ambient noise spectrum level in its frequency band (Fletcher, 1940). Since $S L, T L$ and $N L$ vary, detection range is best understood as a probabilistic distribution. Knowing this distribution is essential for understanding animal behavior because it dictates an animal's maximum possible communication range, which can in turn influence our understanding of how to define the outer limits of a group. Since the group forms the basic unit of analysis for much research into social behavior, accurate knowledge of active space is particularly important.

Previous active space studies have been conducted in a wide variety of species, from red-winged blackbirds, Agelaius phoeniceus, whose songs can be heard 189m away (Brenowitz, 1982) to blue monkeys, Cercopithecus mitis, who have an acoustic range of 870m (Brown, 1989). In marine mammals, Miller (2006) used a hydrophone array to record resident killer whales vocalizing in Johnston Strait and modeled their active space by using an approximation for transmission loss. He found that communication range differed according to the type of vocalization: in calm sea states the active space of shortrange sounds was approximately $5-9 \mathrm{~km}$ but $10-16 \mathrm{~km}$ for long-range vocalizations. Janik (2000) used a similar approach in bottlenose dolphins, Tursiops truncatus, in the Moray Firth, UK, localizing a vocalizing dolphin with a hydrophone array to calculate the source level of whistles and then modeling $T L$ and using published values for ambient noise to predict an active space of 20-25 km. Quintana-Rizzo et al. (2006) used the reported range of bottlenose dolphin whistle source levels and combined this with in-situ measurements of transmission loss in Sarasota Bay, FL, to estimate an active space of $487 \mathrm{~m}$ for a typical 
signature whistle source level. Since both $S L$ and frequency will affect the effective range of a signal and may vary between and within animals, generalizing these values is challenging. In one of the more comprehensive studies on bottlenose dolphins to date, Jensen et al. (2012) used a hydrophone array to estimate $S L$ by localizing individual animals and combined those with separate measurements of background noise and $T L$. In the tropical habitat of Shark Bay, Australia, they reported a median whistle communication range of $750 \mathrm{~m}$ and a maximum range of $5740 \mathrm{~m}$ in optimal conditions.

The wide spread of estimated active space ranges reported above illustrates a couple of key points. First, active space in the ocean is much greater than in air, as a result of reduced sound absorption in seawater (Urick, 1983). Second, a single active space detection range by itself is not useful. Rather, communication range depends on the interaction between the particular signal and the environment through which it is transmitted. Miller's (2006) killer whales had vastly different active space values depending on the type of vocalization used. Even within a single vocalization type, animals can choose to modulate their source levels (Brumm \& Zollinger, 2011), which will in turn affect detection range. Likewise, the transmission loss of the signal depends heavily on environmental factors such as the bathymetry (Urick, 1983). A complete characterization of active space ought to be defined for a particular kind of signal, at a particular source level in a particular environment with a particular level of background noise. Since this level of specificity will not necessarily be possible for naturally behaving animals in a changing environment, it means that neither the producer nor the receiver can predict active space with high precision at any point in time. Rather they must deal with uncertain active space distributions in a probabilistic sense. 
In marine mammals, most of these active space studies have focused on vocalizations such as whistles, but this chapter will concern itself with bottlenose dolphin echolocation clicks. Götz et al. (2006) report that tight groups of traveling rough-toothed dolphins are less prone to echolocating than smaller groups or looser aggregations, suggesting that they may be eavesdropping on the echoes from the sonar clicks of groupmembers. This chapter looks at the detection range not of an echo but of the outgoing click. Although there has been much research on dolphin echolocation in captive settings (e.g. Au, 1993), we know relatively little about how dolphins use their sonar systems in the wild (Au, 2004; Houser et al., 2005; Götz et al., 2006; Jensen et al., 2009). Moreover, the very nature of captive detection experiments trains the dolphins to maximize their $S L \mathrm{~s}$ at values of up to $220 \mathrm{~dB}$ re $1 \mu \mathrm{Pa}$ peak to peak (Au, 1993; Janik, 2000). These $S L$ s may not be typical for dolphins in the wild (Au, 1993; Madsen \& Wahlberg, 2007; Wahlberg et al., 2011) where other factors may affect search behavior or target detection (Jensen et al., 2012), so our understanding of how far a dolphin could keep track of a partner by passively listening to its echolocation clicks could benefit from an in situ exploration of measurements recorded from freely behaving dolphins in their natural habitat. On-animal acoustic tags, like the DTAGs used here (Johnson \& Tyack, 2003), allow us to characterize communication range in terms of distributions taken from naturally behaving animals.

\subsection{DATA USED IN THIS CHAPTER}

Pairs of simultaneously tagged dolphins provide a unique opportunity to explore the transmission of echolocation clicks. I took advantage of the methods already 
described in the preceding chapters, using closely bonded mother-calf pairs of bottlenose dolphins tagged in Sarasota Bay, FL (where Quintana-Rizzo et al. (2006) conducted their study). For each of these pairs, focal and non-focal echolocation clicks were identified on each tag record in order to calculate inter-animal distances. Thus, for each member of a pair, all focal echolocation clicks were extracted as well as the subset of those clicks that were detected on the partner's tag. These detections were used to investigate the potential detection range for an echolocation monitoring strategy. In particular, subsequent calculations in this chapter will be focused on reconstructed tracks from 10 separations across 3 simultaneous tagged pairs of mothers and calves (Table 4.1).

\begin{tabular}{|c|c|c|c|c|c|c|}
\hline Tag ID & Animal ID & Sex & Age & Track Clicks & Tracks & Time(s) \\
\hline $\mathrm{tt13 \_ 130a}$ & FB33 & $\mathrm{F}$ & 31 & 1676 & \multirow{2}{*}{3} & \multirow{2}{*}{1263} \\
\hline $\mathrm{tt} 13 \_130 \mathrm{~b}$ & FB282 & $\mathrm{M}$ & 5 & 1341 & & \\
\hline $\mathrm{tt} 14 \quad 127 \mathrm{a}$ & FB197 & $\mathrm{F}$ & 11 & 2059 & \multirow{2}{*}{4} & \multirow{2}{*}{1179} \\
\hline $\mathrm{tt} 14 \_127 \mathrm{~b}$ & FB243 & F & 2 & 5243 & & \\
\hline tt14_129a & FB185 & $\mathrm{F}$ & 14 & 1445 & & \multirow{2}{*}{632} \\
\hline $\mathrm{tt} 14 \_129 \mathrm{~b}$ & FB249 & $\mathrm{F}$ & 3 & 1020 & & \\
\hline
\end{tabular}

Table 4.1: Summary of simultaneous tag deployments on 3 mother-calf pairs whose reconstructed tracks are used in this chapter

An important consideration to keep in mind is that the data analyzed here are tag data. This means that although the two animals are wearing tags, I am analyzing sound that is recorded on tags, not detected by dolphins. At present this is the best method that exists for wild dolphins; however, it is a slightly different situation from sound passing from one dolphin to another dolphin. Although there is likely much overlap, my inability to detect a sound, such as a click, on the tag record does not imply that the dolphin wearing the tag was not able to detect it. The dolphin's hearing is well adapted to reduce flow noise from water flowing past, that I hear on the tag's hydrophones. Bottlenose dolphin hearing extends across a wide frequency range with best sensitivity between $15 \mathrm{kHz}$ and 
$110 \mathrm{kHz}(\mathrm{Au}, 1993)$. In contrast, the tags used in this thesis have a built in $80 \mathrm{kHz}$ low pass filter, so the dolphins are able to detect frequencies that the tag cannot. More importantly, the measure of click detection used in this chapter was defined by whether or not I could manually detect clicks at very low signal to noise ratios. However, when many clicks from different animals occurred simultaneously, this method needed to bias towards not marking any of the clicks, for incorrectly attributing caller identity would have let to unacceptable spatial errors. Since clicks are not individually distinctive, dolphins may have had trouble detecting them under these circumstances as well. Thus, the click detections on the receiving tag extracted here, are likely good indicators of dolphin click detection, but they may not be perfect. It would be helpful to understand the relationship between the $R L$ at the tag and a dolphin detecting the click.

One way of getting at this distinction would be by using auditory evoked potentials $(A E P)$ that measure the electrical brain activity from the surface of the skin above auditory cortex in response to a stimulus. This technique has been used to construct audiograms and measure hearing sensitivity in dolphins (Nachtigall et al., 2005; Mooney et al., 2006), and a DTAG that can measure $A E P$ s is currently under development that would be capable of measuring $A E P$ data. To match the received level of a click to detection by the receiving dolphin, clicks detected with the current method could be sorted according to $R L$ and I could use the $A E P$ response generated at different $R L \mathrm{~s}$ to estimate the threshold at which dolphins begin to exhibit a brain response to their partner's click. This detection threshold then could be compared to that of my inspection of DTAG data. 


\subsection{COMPARISON BETWEEN ACTIVE AND PASSIVE DETECTION RANGES}

The first thing to explore is a dolphin's active echolocation range. If they remain within that range they may generally be able to locate their partner without having to eavesdrop. However, active echolocation is highly directional, and it may be advantageous to eavesdrop if it is less disruptive to other activities such as an animal's own foraging. Here, I used the following version of the noise-limited active sonar equation (Urick, 1983; Au, 1993) to calculate the active echolocation range of a perfectly on-axis dolphin echolocating on its partner.

$$
D T=S L-2 T L+T S-N+D I r-D
$$

Where $D T$ is the detection threshold of the dolphin, $S L$ the source level, $T L$ the transmission loss, $T S$ a dolphin's target strength, $N$ the ambient noise in the frequency band of the signal, $D I r$ the receiving directivity of the dolphin's hearing, and $D$ is an integration time correction factor. All values are on a logarithmic dB scale. Following recent work on bottlenose dolphin detection thresholds (Au, 2014), I set $D T=0$ to estimate the maximum distance at which the returning echo signal is greater than the ambient noise. For this illustration, a characteristic $N$ in the $20-80 \mathrm{kHz}$ band was measured from a 200s separation from the tags of FB197 and FB248 introduced above. Noise measurements were made from the 0.02 s prior to each click in the separation in

order to reduce the possibility of conflict with the preceding echolocation clicks. Signals were DC corrected and band-pass filtered between 20-80khz to remove low frequency noise and reflect the fact that tags have an inbuilt low-pass filter at $80 \mathrm{kHz}$. Median $N$ across the clicks was $85 \mathrm{~dB}$ RMS re $1 \mu \mathrm{Pa}$. For $S L$, I used an upper bound of the $220 \mathrm{~dB}$ 
reported in captive studies ( $\mathrm{Au}, 1993)$ and a lower value of $177 \mathrm{~dB}$ reported in the wild (Wahlberg et al., 2011). DIr varies with frequency, but I used a value of $15 \mathrm{~dB}$ reported for $60 \mathrm{kHz}$ (Au \& Moore, 1984). TL is doubled in Equation 1 in order to represent the two-way travel. Here I modeled $T L$ according to a spherical spreading equation that is often used for active space calculations in shallow water and absorption as a function of range (Au, 1993; Quintana-Rizzo et al., 2006).

$$
T L=S L-R L=k \log _{10}(R)+\alpha R
$$

Where $R$ is range and $k$ is a geometrical spreading constant often approximated to 20, and the absorption coefficient $\alpha=0.0130$, calculated for a centroid frequency of $60 \mathrm{kHz}$ and a measured temperature of 23 degrees Celsius (Madsen \& Wahlberg, 2007). TS for a dolphin will depend on the particular dolphin, the reflected body part and its orientation, but following $\mathrm{Au}$ (1996), I used $-25 \mathrm{~dB}$ as a reasonable value. A time window correction factor $(D)$ accounts for the difference between the duration of the $S L$ values $(15-20 \mu \mathrm{s}$,

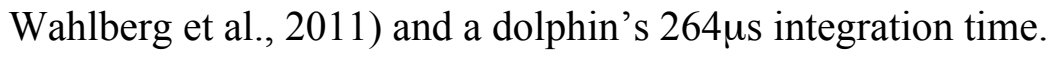

$$
D=10 \log _{10}\left(\frac{18 \mu s}{264 \mu s}\right)
$$

Solving for $R$ calculated a maximum active echolocation detection range of $384 \mathrm{~m}$ for a $S L$ of $220 \mathrm{~dB}$ and $53 \mathrm{~m}$ for a $S L$ of $177 \mathrm{~dB}$. Since animals modulate their source levels, these values are best understood as upper and lower ranges that reflect an open water distribution rather than absolute numbers. 
Now, moving from active echolocation to the case where a dolphin is passively listening to the sonar clicks of its conspecific and assuming on-axis clicks, I used the passive sonar equation:

$$
D T=S L-T L-N+D I r-D
$$

Note that in this case there is no target strength, for no reflection is occurring. $T L$ appears only once to represent one-way travel. Setting $D T=0$ and solving for $R$ as above yields a detection range of approximately $4957 \mathrm{~m}$ for a $S L$ of $220 \mathrm{~dB}$ and $2194 \mathrm{~m}$ for a $S L$ of $177 \mathrm{~dB}$. Thus, a strategy that passively listens to conspecific echolocation clicks can do so at a much greater range than an active echolocation approach if the clicking dolphin is pointing towards the listening dolphin. The average maximum separation distance was $96 \mathrm{~m}$ for simultaneously tagged mother-calf pairs and $250 \mathrm{~m}$ for male-male pairs of dolphins. Therefore, the animals' separation distances overlap with the active range, but remain well within the passive one, suggesting that passive strategy may be a useful.

\subsection{EMPIRICAL ESTIMATION OF MONITORING DISTANCE}

\subsubsection{Addressing directivity of dolphin biosonar}

Studies into the biosonar system of captive dolphins have shown that their echolocation clicks are highly directional signals with a three $\mathrm{dB}$ beam width of only 10 degrees in the horizontal or vertical planes (Au, 1993). Thus, if the clicking dolphin is aiming directly at the receiving animal, the level received by the receiving dolphin may closely reflect the on-axis $S L-T L$. However, any deviation from that on-axis orientation 
will result in the actual signal transmitted in the direction of the receiver being reduced as a function of the beam pattern.

To take directivity into account requires information about the angle between the axis of the clicking animal and the receiving animal. Focusing on 10 separation and reunion events, I recreated animal tracks from the tag records corrected for the interanimal range in order to calculate that angle. Frants Jensen developed the method to create the tracks as part of a larger project.

To reconstruct underwater tracks for each dolphin, I used pitch, roll and heading from the tag's inertial sensors. Dead-reckoning the tracks from the orientation sensors and using flow noise to estimate speed without any optimization would have led to large errors. Therefore, I estimated parameters for the initial location, heading error, and the intercept and slope of a linear fit of speed as a function of flow noise. The best value of one parameter will depend on all of the others, so a 4D grid search was used over a wide set of parameters to find the optimal values. These values were the ones that minimize the sum of the squared error (SSE) between the reconstructed tracks and the separation range empirically calculated from the detected clicks. This approach created more accurate estimates of the individual animal tracks. From the time of each outgoing click, I calculated the vector angle between the position of the clicking animal and of the receiving animal. This vector was combined with the heading measurements to calculate the off-axis angle between the clicking animal and the receiver. 

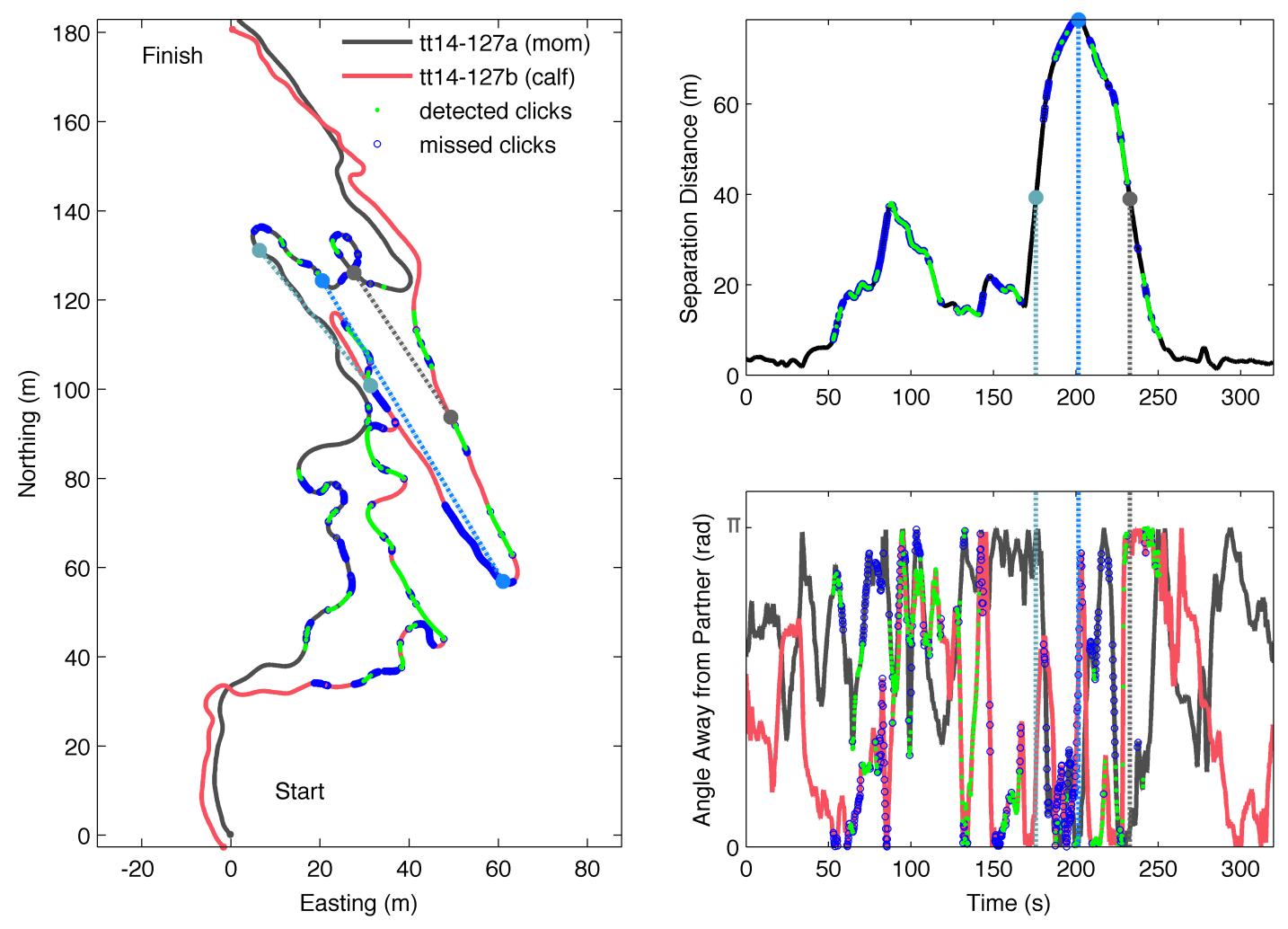

Figure 4.1: Left, reconstructed and corrected track for a separation between tt14_127a (mother, black) and tt14_127b (calf, red). Emitted clicks are green if detected, blue if missed. Upper right shows inter-animal separation distance vs time. Lower right shows absolute off-axis angle with respect to its partner with 0 being pointing directly towards it. Dotted lines connect 3 points at the same time across both tracks corresponding to $50 \%$ and $100 \%$ separation.

Figure 4.1 illustrates the relationship between reconstructed tracks for $t t 14 \_127 \mathrm{ab}$ and inter-animal separation distance. Over this track, the mother (black) maintained a fairly constant slow speed, but the calf (red) accelerated away during the large separation.

I limited calculations to clicks recorded when the animals were greater than $7 \mathrm{~m}$ apart since any error in the tracks would be exacerbated in the angle calculations at close distances. I chose $7 \mathrm{~m}$ to match the minimum separation distance used in Chapter Three. With the separation distance and the relative angle, I then back-calculated the apparent 
source level $(A S L)$ from $R L$ as a function of off-axis angle (Møhl et al., 2000; Zimmer et al., 2005; Madsen \& Wahlberg, 2007).

$$
A S L=R L+T L=R L+k \log _{10}(R)+\alpha R
$$

As above, $T L$ was modeled as spherical spreading with a loss constant of $k=20$ and an absorption constant of $\alpha=0.0130$. Figure 4.2 shows plots of $A S L$ versus off axis angle for outgoing clicks from the three mother-calf pairs.
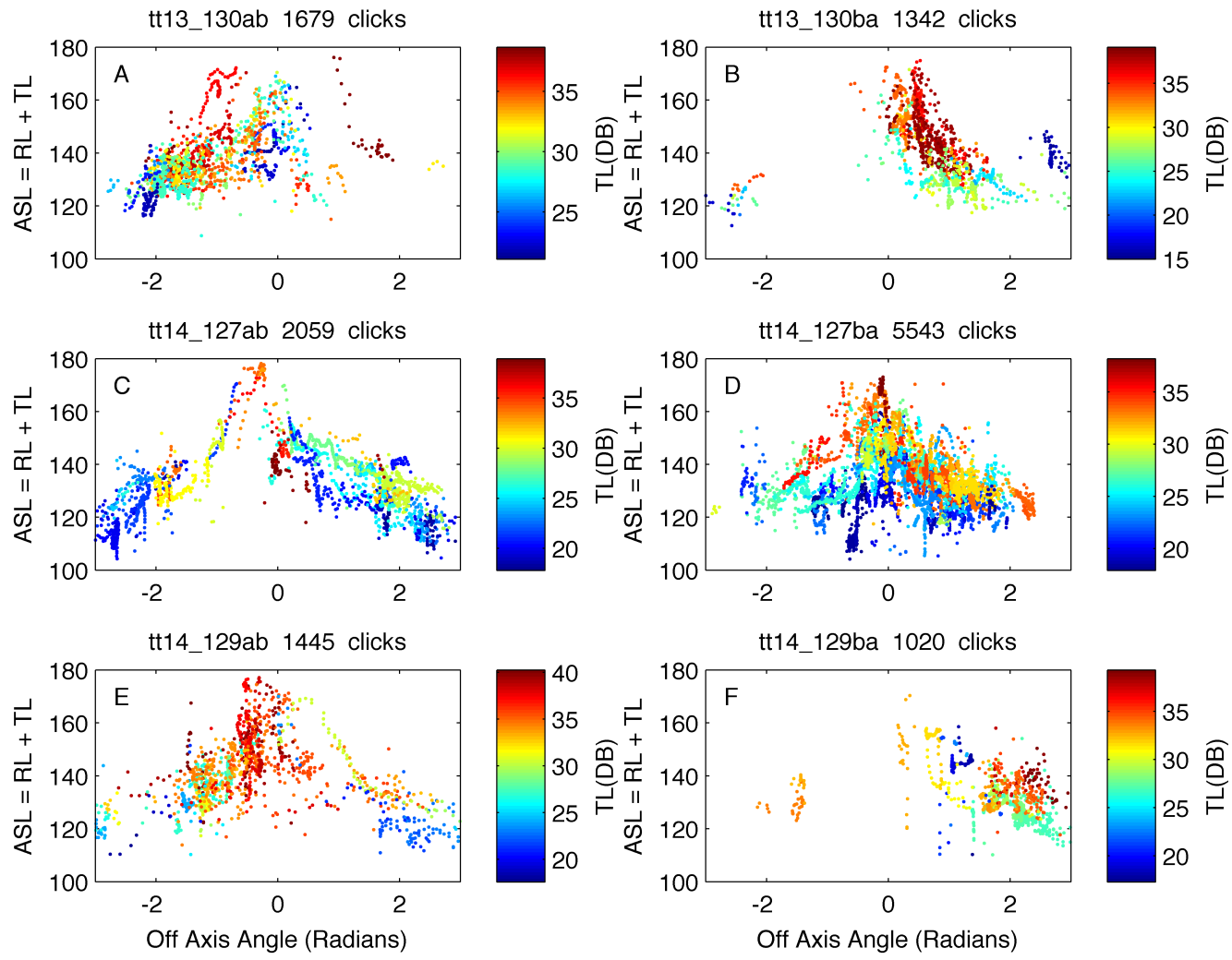

Figure 4.2: Back-calculated apparent source level ( $A S L)$ vs off-axis angle for outgoing clicks from 3 sets of partnered animals (AB, CD, EF). Mothers are shown on the left and calves on the right. Points are colorcoded according to transmission loss $(T L)$. Clicks are aggregated across 3 $(\mathrm{AB}), 4(\mathrm{CD})$ and $3(\mathrm{EF})$ separation and reunion tracks. 
Another, perhaps more intuitive way of visualizing off-axis angle is on a polar plot.

Figure 4.3 shows polar representations of $A S L$ and off-axis angle for the same mothercalf pairs.
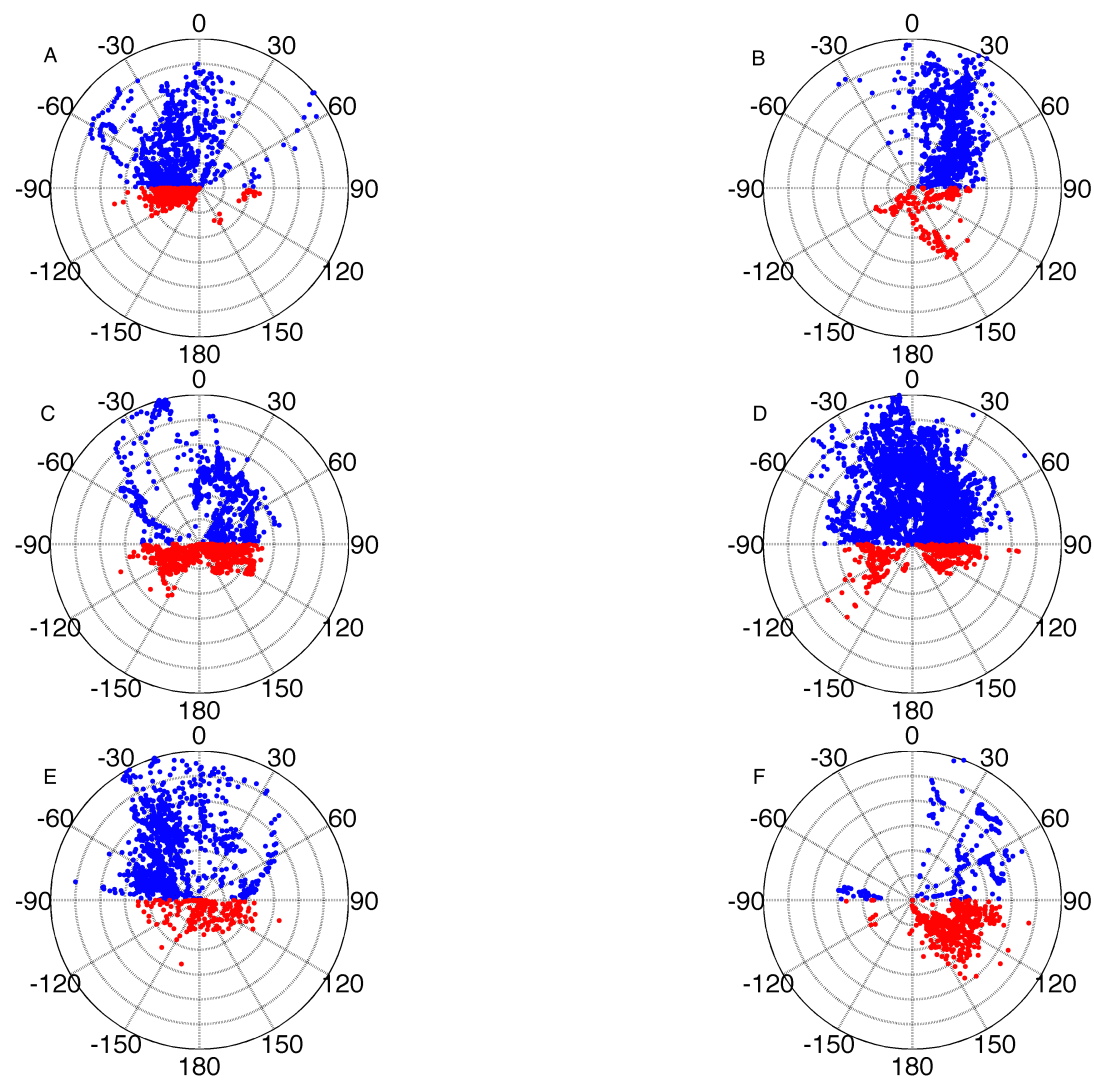

Figure 4.3: Polar plots of horizontal off-axis angle (in degrees) for each detected outgoing click emitted by the 3 sets of partnered animals shown in Figure 3 ( $\mathrm{AB}, \mathrm{CD}, \mathrm{EF})$. Zero degrees would be perfectly on-axis. Apparent source level $(A S L)$ is shown in decreasing $10 \mathrm{~dB}$ concentric contours, with the outer contour representing the maximum recorded $A S L$. Clicks emitted while pointing towards the receiving animal $\left(-90^{\circ}\right.$ to $\left.+90^{\circ}\right)$ are colored blue. Clicks emitted while pointing away $\left(+90^{\circ}\right.$ to $\left.-90^{\circ}\right)$ are colored red.

The three distributions generally fit a beam pattern as described by the toothed whale biosonar literature with $A S L$ being higher around the acoustic axis and falling off as angle increases (Au, 1993). However, in some cases they were incomplete, and there 
are a few caveats. First, head movement may cause a difference between the acoustic and body axes, so there may be a certain amount of offset (Zimmer et al., 2005). This headscanning behavior is evident in the tag recordings, where we routinely see changes in angle of incidence of outgoing clicks from a tagged animal in the order of $\pm 20^{\circ}$. Incorporating the angle of arrival between the hydrophones of the clicking dolphin's signal at its own tag (Johnson et al., 2009) can mitigate this offset, but it is not a perfect solution. Likewise, although the tracks corrected by the known inter-animal distances are much better than uncorrected ones, they will still incorporate some error in the relative location of tagged individuals. Finally, I simplified the estimates of off-axis angle as horizontal off-axis angle. This ignores the fact that animals may be searching for, or tracking, prey throughout the water column and perhaps especially near the bottom. Incorporating pitch into the calculation did not noticeably improve the patterns. This is likely because our estimate of pitch from tagged animals itself has a relatively large margin of error, but partly also because the pitch of the dolphin may not accurately represent the axis of the sonar beam. My estimate of $A S L$ does not rely on $A O$, so no correction is required for the difference between the $A O$ recorded by the tag worn on the dolphin's back and its actual SL. While the patterns generally resemble the beam shape of a circular piston, which is commonly used to model beam patterns, the high variance in on-axis $A S L$ prevents us from making a complete fit of each dolphin's echolocation beam. However, despite the incomplete beam, the maximum $A S L$ values will approximate $S L$ when close to on-axis. The back-calculated $A S L$ values found ranged from $170-180 \mathrm{~dB}$, lower than the maximum values reported for captive animals and more similar to the values reported for free-ranging dolphins (Wahlberg et al., 2011). 


\subsubsection{Empirical distribution of detection range for free-swimming dolphins}

I used the measured values of $A S L$ from the perspective of an eavesdropping conspecific to model the range of possible detection distances that a dolphin encounters.

Figures 4.4 extends our previous $R L$ to $A S L$ calculation in order to find the maximum distance at which simulated $R L$ could be heard above the noise recorded on the receiver's tag at that point in time according to the rearranged passive sonar equation:

$$
N L=R L=A S L-T L=A S L-k \log _{10}(R)-\alpha R
$$

Where $k=20$ and $\alpha=0.0130$ as before.
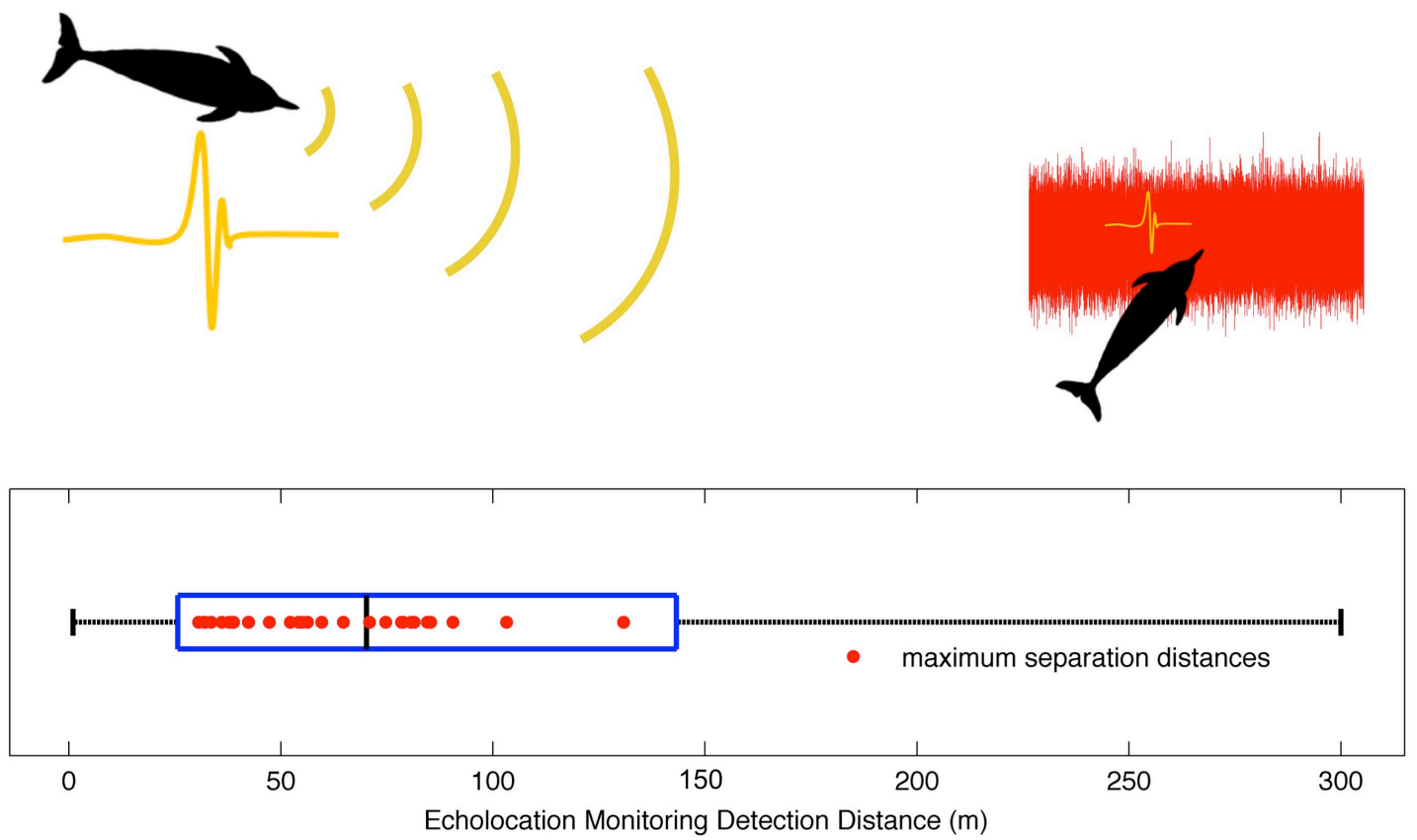

Figure 4.4: Pairs of mother and calf dolphins are often at separation distances where they could use an echolocation monitoring strategy to track each other, but they also travel beyond that range. The maximum potential echolocation monitoring range is a function of the source level of the outgoing click, the transmission loss as it travels and the noise over which it must be detected at the receiver. The blue boxplot shows the distribution of maximum ranges at which each of the 13,082 emitted clicks would have been able to be detected above the noise on the receiver's tag at that point in time. Measured peak separations from mothers and calves are overlaid on the box plot as red dots. 
The intersections of these simulated $R L$ values with the noise floor recorded on the receiver's tag represent a distribution of the potential detection ranges that Sarasota Bay dolphins encounter when swimming in their natural environment. These distributions illustrate a measure of the prospective range for a potential echolocation monitoring strategy and show that the maximum separation distances of mother-calf dolphins are often within the potential range of an echolocation monitoring tracking strategy but also separate beyond it at times. It is important to note that the dolphin's ear and brain may be able to detect clicks at larger ranges than I was able to do from DTAG recordings, but I think it unlikely that the tag and my detector performed better than a dolphin as a receiver.

\subsubsection{Probability distributions of maximum detection range}

A complementary way of looking at these simulated $R L$ 's would be to consider the probability distributions of maximum detection ranges. Before randomly sampling from the distributions of $A S L$ and noise recorded from the clicking animal just prior to each click, it is necessary to check for any potential relationship between $A S L$ and noise. An example of a potential relationship would be the Lombard effect, first noticed by Etienne Lombard in 1911 that human speakers (and by extension animals) involuntarily increase $S L$ in the presence of background noise (Brumm \& Zollinger, 2011). Figure 4.5 plots $A S L$ vs noise for the tt14_127ab pair to look for any relationship. 

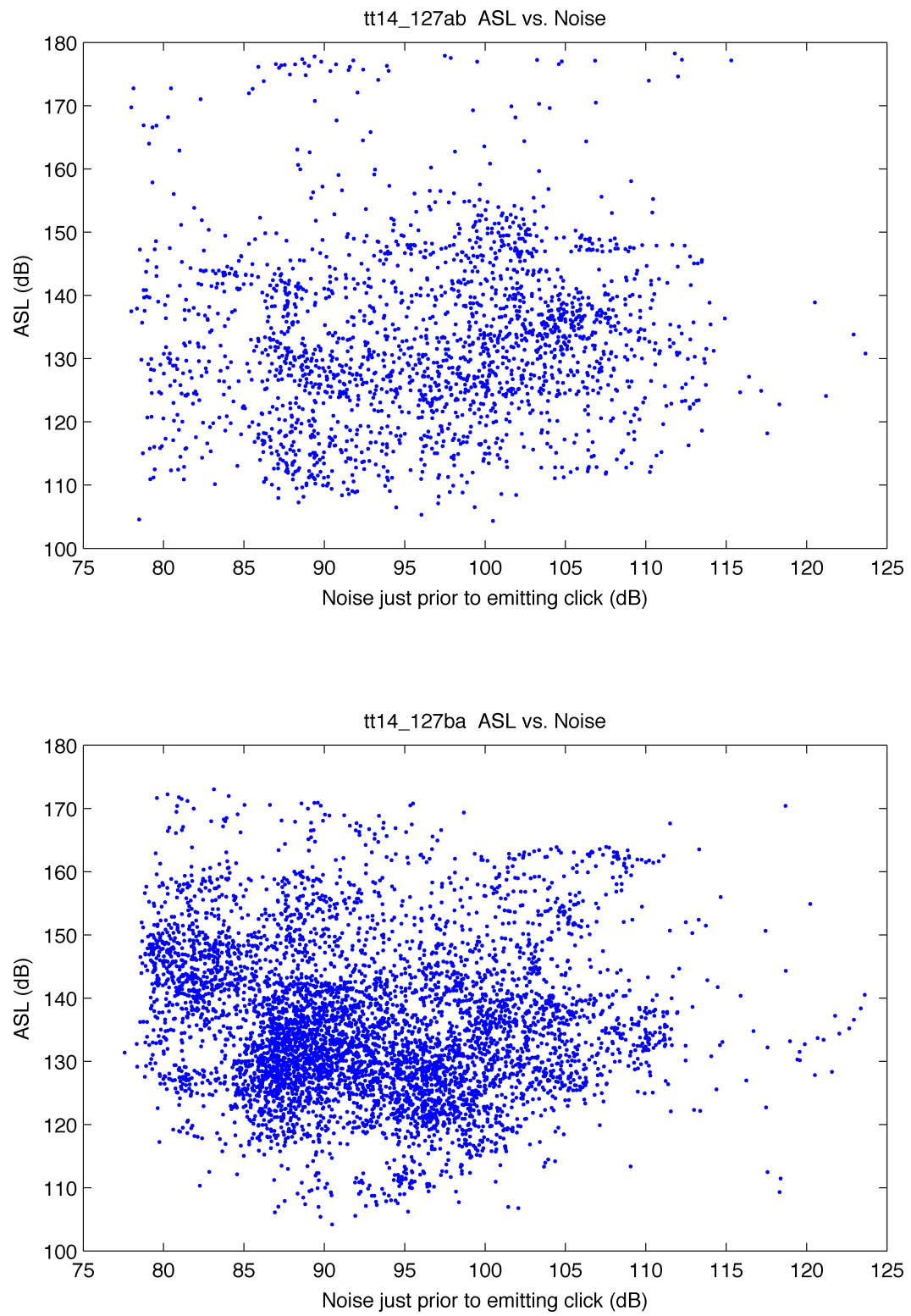

Figure 4.5: Apparent source level $(A S L)$ vs noise just prior to emitting a click for tt14_127a (top) and tt14_127b (bottom).

Although Figure 4.5 doesn't show a strong pattern in the scatter, to mitigate any potential relationship I will pair each $A S L$ with its own noise measurement when calculating maximum detection ranges rather than sampling randomly. Given the directionality of 
dolphin sonar, I would expect that the distributions would be different for times when the animals are moving towards (blue dots in Figures 4.3) and away from (red dots in Figures 4.3) their partner. Figure 4.6 shows the probability distribution of maximum detection ranges that an animal is likely to encounter, calculated using kernel density estimation.
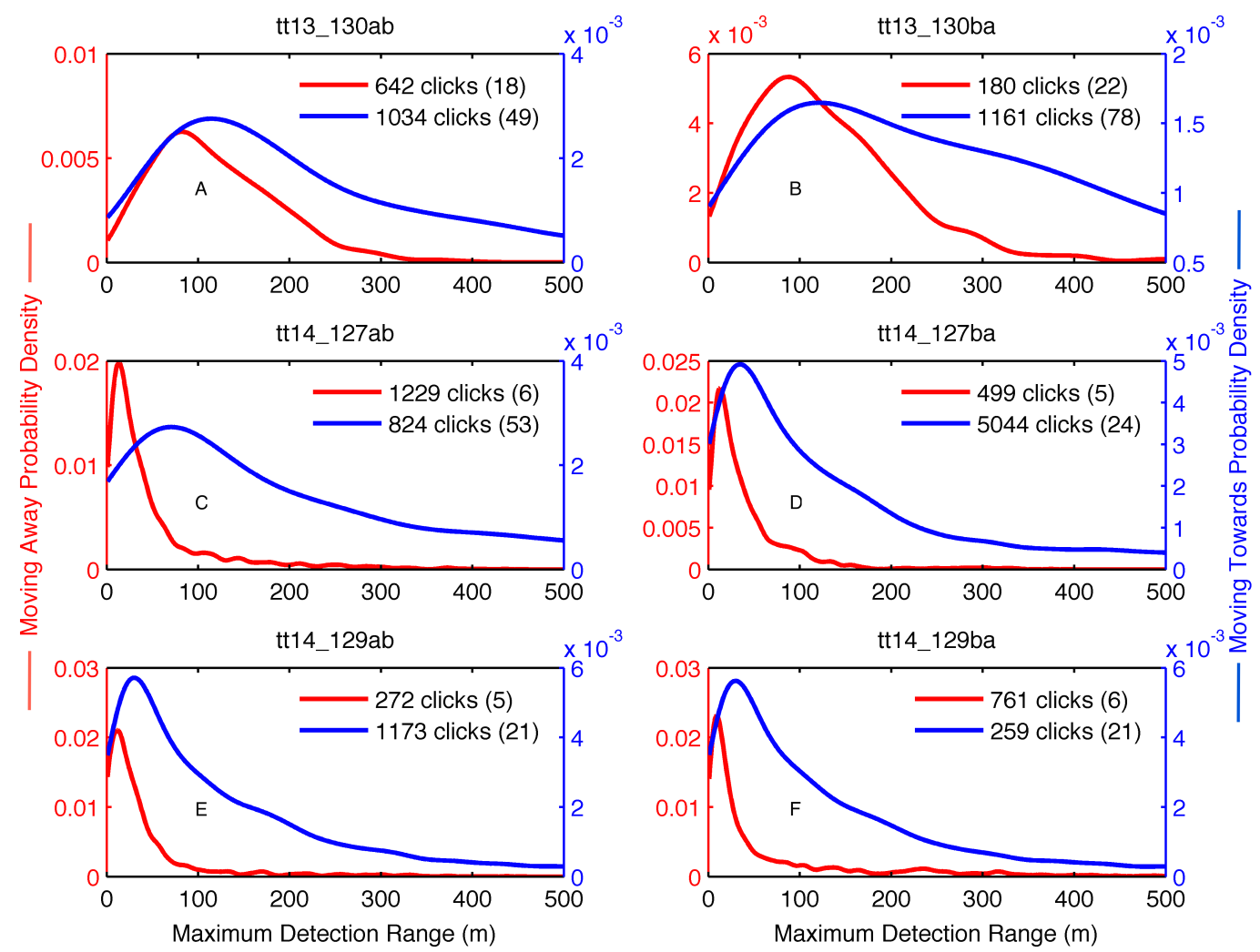

Figure 4.6: Probability density functions (PDF) of maximum detection ranges for clicks emitted by each dolphin. PDF estimated from distribution of apparent source levels $(A S L)$ and noise prior to emitted clicks as in Figure 4.5. Different functions are shown for clicks while pointing away from (red) and towards (blue) its partner. The smoothing for these functions used a Gaussian kernel with bandwidth chosen based on the number of clicks shown in parentheses.

The PDF distributions of maximum detection range (Figure 4.6) illustrate the expected differences between moving towards and moving away. In all cases, the most likely 
maximum detection range is greater when the animals are moving towards each other than away. This makes intuitive sense given the directivity of a dolphin's biosonar.

\subsubsection{Probability of click detection at a given range}

This section explores how the probability of an emitted click being detected varies with range. As discussed above, my analysis detects every outgoing click and the subset of those that were detected on the partner tag. The processing methods were developed by Mark Johnson and Frants Jensen (Johnson et al., 2015). Briefly, echolocation clicks were identified and manually categorized as produced by the tagged dolphin (focal) or other dolphin (non-focal) on each tag record based on the angle of arrival and lowfrequency energy. Once this first pass had identified and sorted the clicks, they were filtered through a supervised click detector that relies on amplitude and angle of arrival to fine-tune classification. Then, the emitted clicks were visually identified on the partner receiving tag after accounting for a time offset stemming from travel distance. The signal-to-noise ratio of the received clicks was often quite low, and it was only possible to identify a subset. Moreover, there is a high cost to mis-identifying a received click in resulting tracks and calculations, so my detection was biased towards being conservative.

In addition to these detected and missed clicks, the range at each point in time allowed detection probability in increasing range bins to be calculated as follows:

$$
p(\text { detection })=\frac{\text { number of detected clicks }}{\text { number of emitted clicks }}
$$

Again, data were split into categories where the animals were moving away from and towards their partners. 

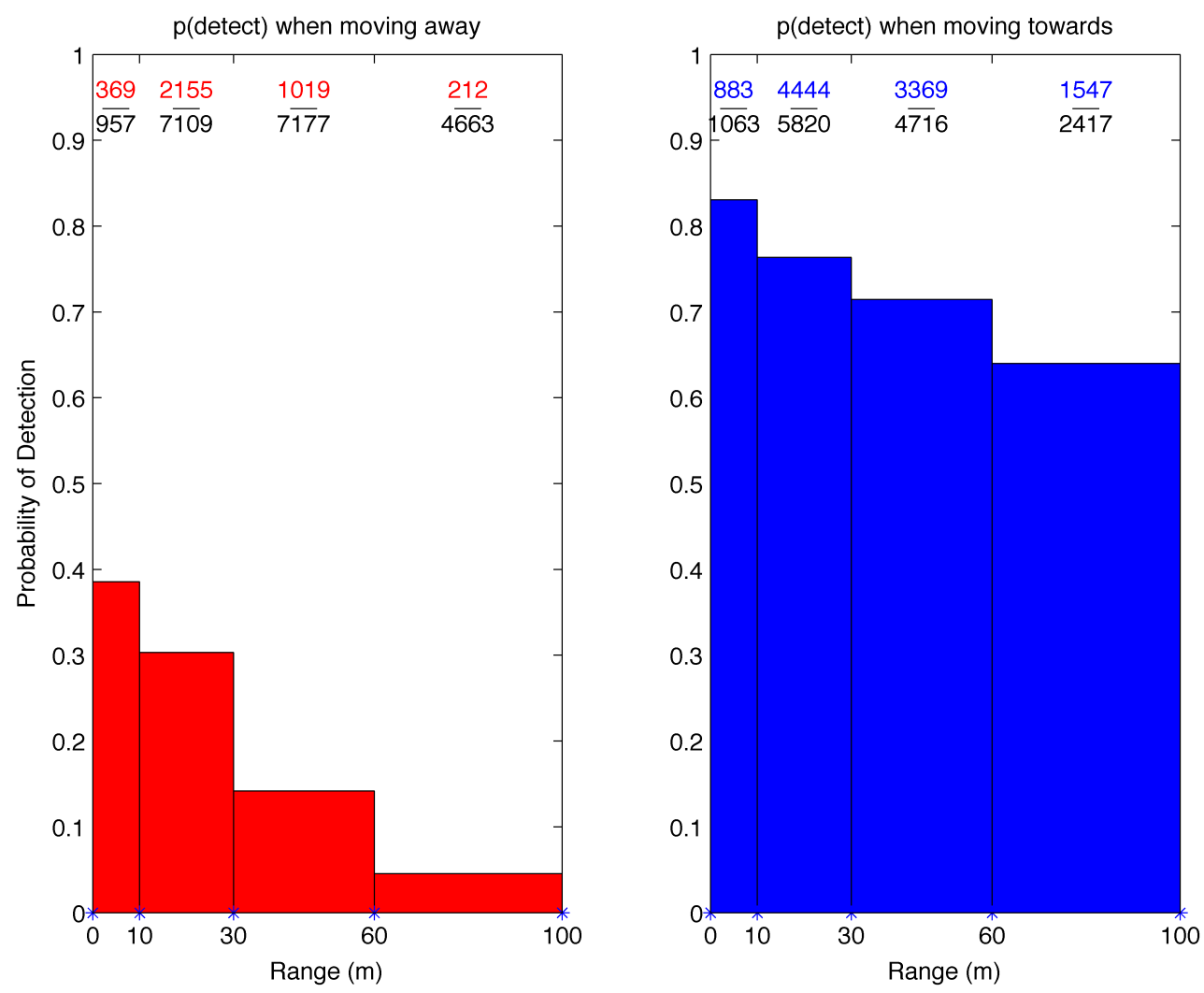

Figure 4.7: Probability of an emitted click being detected at a given range while the clicking dolphin is moving away and moving towards its partner. Clicks are aggregated across all animals and separations. Probabilities were calculated in bins of increasing size. The number of detected (blue and red) and emitted clicks (black) in each bin are shown at the top of the plots.

The distribution of the probability of an emitted click being detected by the tag on the animal's partner (Figure 4.7) showed the pattern I would expect: when the clicking dolphin was moving away from its partner, emitted clicks were generally detected at close ranges, but probability of detection decreased at greater separation ranges. When the clicking dolphin was moving towards the partner, probability of detection was relatively high at all ranges. Note that the bin size increases with range to reflect 
increasing $T L$ with range. Looking at each animal individually showed the same pattern as the aggregate.

\subsection{EFFECT OF ECHOLOCATION MONITORING ON THE PROBABILITY OF EMITTING SIGNATURE WHISTLES}

Chapter Three modeled the probability of a signature whistle being produced in a separation-reunion event as the square of percent distance and whether or not the animal was in a separation or reunion. The data provided evidence for signature whistles representing a dolphin's motivation to reunite. Combining this hypothesis with the idea of echolocation monitoring, an increase in time since an animal last detected its partner's echolocation click might increase its motivation to reunite and the probability of emitting a signature whistle.

To test this idea, I added this parameter to the model from Chapter Three to see if it improved the fit. I included a variable at each point in time that captured the number of seconds since the focal tag detected a click from the partner animal (silence variable).

After repeating variable selection, the re-fitted logistic regression model is shown below.

$$
\log \left(\frac{p(s w)}{1-p(s w)}\right)=\beta_{0}+\beta_{1} \% \text { Dist }^{2}+\beta_{2} \% \text { Dist }+\beta_{3} \text { Separation }+\beta_{4} \text { Silence }
$$

Table 4.2 reports model coefficients and p-values.

\begin{tabular}{r|rrrr} 
sw & \multicolumn{1}{|c}{ Coef. } & Std. Err. & \multicolumn{1}{c}{$\mathbf{z}$} & $\mathbf{p}$ \\
\hline Intercept $^{2}$ & -5.1486379 & 0.8458416 & -6.087 & $1.15 \mathrm{E}-09$ \\
Percent Distance $^{2}$ & 0.0008861 & 0.0002764 & 3.205 & 0.00135 \\
Percent Distance $^{-0.0865906}$ & 0.0333091 & -2.600 & 0.00933 \\
In Separation & -0.7985785 & 0.3753467 & -2.128 & 0.03337 \\
Silence & 0.0061237 & 0.0027191 & 2.252 & 0.02432 \\
\hline
\end{tabular}

Table 4.2. Summary of re-fitted binomial logistic regression model showing the significant improvement coming from adding the silence variable (shaded). 
After re-fitting our model, this new silence variable made a significant contribution with a p-value of 0.024 and a positive coefficient of 0.006 . This coefficient corresponds to an odds ratio of 1.006, meaning that for every second that an animal had not detected a click from its partner, its odds of producing a signature whistle increased by a factor of 1.006 .

Thus, adding a variable that accounted for time since last detected click provided a measurable improvement to our model of probability of signature whistle during a separation-reunion event. The model improvement is consistent with animals passively eavesdropping on their conspecifics' echolocation clicks to monitor their locations, and having increasing motivation to whistle the longer they have not been able to hear their partners echolocation clicks.

\subsection{CONCLUSION}

This chapter provides evidence that bottlenose dolphins may use an echolocation monitoring strategy to keep track of closely bonded conspecifics. First, I estimated upper and lower bounds on the passive detection range for bottlenose dolphins using previously reported estimates of their biosonar source levels. I then showed that source levels used in the wild are lower than maximum estimates in the literature, and demonstrated that passive detection range in the wild should be on the order of $\sim 100 \mathrm{~m}$, with lower passive detection range as animals are moving apart compared to when they are moving towards each other. I proceeded to show that typical separation distances between mother-calf pairs fall within these limits of passive monitoring range, but that they also separate beyond the passive monitoring range. Finally, I showed that, in a separation and reunion context, the amount of time since an animal was last able to use an echolocation 
monitoring strategy helped explain its probability of producing a signature whistle, consistent with the idea that passively listening to a conspecifics biosonar clicks may reduce a dolphin's motivation to produce cohesion calls.

Taken together, these lines of evidence describe mother-calf pairs of dolphins that often remain within a range where they can hear each other's echolocation clicks, despite individual foraging needs that drive them to separate. This suggests that inter-animal distance may not always be sufficient to completely characterize group cohesion: if the dolphins can still occasionally keep track of each other through echolocation monitoring, it may not be completely correct to think of them as separated. In turn, echolocation monitoring may help explain the many separations and reunions that were mediated without any signature whistles. Throughout a separation this suggests that mother and calf pairs of bottlenose dolphins can solve much of the tracking problem through echolocation monitoring, without requiring them to pause their foraging or exploration to signal with whistles. At the beginning of a separation they can likely use echolocation monitoring to track each other passively, then they may separate beyond a range where this is viable because they will probably be pointing away from each other. At this point signature whistles may come into play to signal a reunion, and once they face towards each other they will likely be able to use echolocation monitoring again to mediate cohesion. 


\subsection{REFERENCES}

Au, W. W. (1996). Acoustic reflectivity of a dolphin. Journal of the Acoustical Society of America, 99(6), 3844-3848.

$\mathrm{Au}, \mathrm{W}$. W. (2014). Dolphin biosonar target detection in noise: Wrap up of a past experiment. Journal of the Acoustical Society of America, 136(1), 9-12.

Au, W. W. L. (1993). The Sonar of Dolphins. [electronic resource]: New York, NY : Springer New York, 1993.

Au, W. W. L. (2004). Echolocation signals of wild dolphins. Acoustical Physics, 50(4), 454-462.

Au, W. W. L., \& Moore, P. W. B. (1984). Receiving beam patterns and directivity indexes of the atlantic bottlenose dolphin Tursiops truncatus. Journal of the Acoustical Society of America, 75(1), 255-262.

Brenowitz, E. A. (1982). The active space of red-winged blackbird song. Journal of Comparative Physiology, 147(4), 511-522.

Brown, C. H. (1989). The active space of blue monkey and grey-cheeked mangabey vocalizations. Animal Behaviour, 37, 1023-1034.

Brumm, H., \& Zollinger, S. A. (2011). The evolution of the Lombard effect: 100 years of psychoacoustic research. Behaviour, 148(11-13), 1173-1198.

Connor, R. C., Wells, R. S., Mann, J., \& Read, A. J. (2000). The Bottlenose Dolphin. In J. Mann, R. C. Connor, P. L. Tyack \& H. Whitehead (Eds.), Cetacean Societies: Field studies of whales and dolphins (pp. 91-126). Chicago: Univ. Chicago Press.

Cvikel, N., Berg, K. E., Levin, E., Hurme, E., Borissov, I., Boonman, A., .. . Yovel, Y. (2015). Bats aggregate to improve prey search but might be impaired when their density becomes too high. Current Biology, 25, 206-211.

Fletcher, H. (1940). Auditory Patterns. Reviews of Modern Physics, 12(1), 47.

Götz, T., Verfuß, U. K., \& Schnitzler, H.-U. (2006). 'Eavesdropping'in wild roughtoothed dolphins (Steno bredanensis)? Biology Letters, 2(1), 5-7.

Houser, D., Martin, S. W., Bauer, E. J., Phillips, M., Herrin, T., Cross, M., . . Moore, P. W. (2005). Echolocation characteristics of free-swimming bottlenose dolphins during object detection and identification. Journal of the Acoustical Society of America, 117(4), 2308-2317.

Janik, V. M. (2000). Source levels and the estimated active space of bottlenose dolphin (Tursiops truncatus) whistles in the Moray Firth, Scotland. Journal of Comparative Physiology A, 186, 673-680.

Janik, V. M. (2009). Acoustic communication in delphinids. In M. Naguib, V. Janik, N. Clayton \& K. Zuberbuhler (Eds.), Advances in the Study of Behavior (Vol. 40, pp. 123-157): Academic Press.

Jensen, F. H., Beedholm, K., Wahlberg, M., Bejder, L., \& Madsen, P. (2012). Estimated communication range and energetic cost of bottlenose dolphin whistles in a tropical habitat. Journal of the Acoustical Society of America, 131(1), 582-592.

Jensen, F. H., Bejder, L., Wahlberg, M., \& Madsen, P. T. (2009). Biosonar adjustments to target range of echolocating bottlenose dolphins (Tursiops sp.) in the wild. Journal of Experimental Biology, 212(8), 1078-1086. doi: 10.1242/jeb.025619 
Johnson, M., de Soto, N. A., \& Madsen, P. T. (2009). Studying the behaviour and sensory ecology of marine mammals using acoustic recording tags: a review. Marine Ecology Progress Series, 395, 55-73. doi: 10.3354/meps08255

Johnson, M., Jensen, F. H., Miller, P., Madsen, P. T., de Soto, N. A., \& Tyack, P. L. (2015). Measuring the separation distance and relative location of animals using sound recording tags. Behavioral Ecology and Sociobiology, Submitted.

Johnson, M. P., \& Tyack, P. L. (2003). A digital acoustic recording tag for measuring the response of wild marine mammals to sound. IEEE Journal of Oceanic Engineering, 28(1), 3-12.

Madsen, P. T., \& Wahlberg, M. (2007). Recording and quantification of ultrasonic echolocation clicks from free-ranging toothed whales. Deep-Sea Research Part I Oceanographic Research Papers, 54(8), 1421-1444.

Marten, K., \& Marler, P. (1977). Sound transmission and its significance for animal vocalization. Behavioral Ecology and Sociobiology, 2(3), 271-290.

Miller, P. (2006). Diversity in sound pressure levels and estimated active space of resident killer whale vocalizations. Journal of Comparative Physiology A: Neuroethology, Sensory, Neural, and Behavioral Physiology, 192(5), 449-459. doi: 10.1007/s00359-005-0085-2

Møhl, B., Wahlberg, M., Madsen, P. T., Miller, L. A., \& Surlykke, A. (2000). Sperm whale clicks: Directionality and source level revisited. Journal of the Acoustical Society of America, 107(1), 638-648.

Mooney, T. A., Nachtigall, P. E., \& Yuen, M. M. (2006). Temporal resolution of the Risso's dolphin, Grampus griseus, auditory system. Journal of Comparative Physiology A, 192(4), 373-380.

Nachtigall, P. E., Yuen, M. M., Mooney, T. A., \& Taylor, K. A. (2005). Hearing measurements from a stranded infant Risso's dolphin, Grampus griseus. Journal of Experimental Biology, 208(21), 4181-4188.

Quintana-Rizzo, E., Mann, D. A., \& Wells, R. S. (2006). Estimated communication range of social sounds used by bottlenose dolphins (Tursiops truncatus). Journal of the Acoustical Society of America, 120(3), 1671-1683.

Urick, R. J. (1983). Principles of Underwater Sound: Peninsula, Los Altos.

Wahlberg, M., Jensen, F. H., Soto, N. A., Beedholm, K., Bejder, L., Oliveira, C., . . . Madsen, P. T. (2011). Source parameters of echolocation clicks from wild bottlenose dolphins (Tursiops aduncus and Tursiops truncatus). Journal of the Acoustical Society of America, 130(4), 2263-2274.

Zimmer, W. M. X., Johnson, M. P., Madsen, P. T., \& Tyack, P. L. (2005). Echolocation clicks of free-ranging Cuvier's beaked whales (Ziphius cavirostris). Journal of the Acoustical Society of America, 117(6), 3919-3927. 


\section{Chapter 5: A 3D stereo camera system for precisely positioning animals in space and time}


This chapter was published originally published as

Macfarlane, N. B. W., Howland, J. C., Jensen, F. H., \& Tyack, P. L. (2015). A 3D stereo camera system for precisely positioning animals in space and time. Behavioral Ecology and Sociobiology, 1-9. doi: 10.1007/s00265-015-1890-4

It is reprinted here with permission from Springer Science and Behavioral Ecology and Sociobiology 
TITLE: A 3D Stereo Camera System for Precisely Positioning Animals in Space and Time

Authors:

Nicholas B. W. Macfarlane ${ }^{(1,2)}$

Jonathan C. Howland ${ }^{(3)}$

Frants H. Jensen ${ }^{(1,4)}$

Peter L. Tyack ${ }^{(1,5)}$

${ }^{1}$ Department of Biology, Woods Hole Oceanographic Institution, Woods Hole, MA 02543, USA

${ }^{2}$ Department of Biology, Massachusetts Institute of Technology, Cambridge, MA 02139, USA

${ }^{3}$ Department of Applied Ocean Physics and Engineering, Woods Hole Oceanographic Institution, Woods Hole, MA 02543, USA

${ }^{4}$ Department of Ecology and Evolutionary Biology, Princeton University, Princeton, NJ 08540, USA

${ }^{5}$ Sea Mammal Research Unit, Scottish Oceans Institute, Department of Biology, University of St. Andrews, Fife, KY16 8LB, UK

Corresponding author:

Nicholas Macfarlane 266 Woods Hole Rd MS 50

Woods Hole, MA 02543

USA

nicholas@whoi.edu

$+1.508 .289 .3254$ 


\section{Keywords}

- Photogrammetry

- Group cohesion

- Collective behaviour

- Geo-location

- Range-finding 


\subsection{ABSTRACT}

Here, we describe a portable stereo camera system that integrates a GPS receiver, an attitude sensor, and 3D stereo photogrammetry to rapidly estimate the position of multiple animals in space and time. We demonstrate the performance of the system during a field test by simultaneously tracking the individual positions of six long-finned pilot whales, Globicephala melas. In shore-based accuracy trials, a system with a $50-\mathrm{cm}$ stereo baseline had an average range estimation error of $0.09 \mathrm{~m}$ at a $5-\mathrm{m}$ distance increasing up to 3.2 at $50 \mathrm{~m}$. The system is especially useful in field situations where it is necessary to follow groups of animals travelling over relatively long distances and time periods whilst obtaining individual positions with high spatial and temporal resolution (up to $8 \mathrm{~Hz}$ ). These positions provide quantitative estimates of a variety of key parameters and indicators for behavioural studies such as inter-animal distances, group dispersion, speed and heading. This system can additionally be integrated with other techniques such as archival tags, photo-identification methods or acoustic playback experiments to facilitate fieldwork investigating topics ranging from natural social behaviour to how animals respond to anthropogenic disturbance. By grounding observations in quantitative metrics, the system can characterize fine-scale behaviour or detect changes as a result of disturbance that might otherwise be difficult to observe.

\subsection{INTRODUCTION}

Many animals live in groups that provide important benefits to the individual, such as decreased predation risk and lower cost of movement (Hamilton 1971; Krause and Ruxton 2002). These benefits often depend on the relative position of an individual within a group (Sumpter et al. 2008) and the behavior of other group members (Conradt and Roper 2003; Bode et al. 2011). Realizing the benefits of a group often requires mechanisms for maintaining spatial cohesion (Krause and Ruxton 2002) and for reuniting with the group after temporary separations (Da Cunha and Byrne 2009).

Many theoretical studies have modelled how simple behaviours by individual group members with differing motivations (Conradt and Roper 2000) can give rise to remarkably complex collective movements (Gueron and Levin 1993; Parrish and Hamner 1997). These studies indicate the importance of an individual's relative spatial position 
for many aspects of collective motion ranging from the impact of social networks (Bode et al. 2011) and decision-making structures (Conradt and Roper 2003; Conradt and Roper 2010) to transferring information throughout the group (Couzin et al. 2005; Sumpter et al. 2008). Until recently, empirical studies have lagged behind theory because they lacked the precision and accuracy to collect spatial data in fast-moving groups of animals (Nagy et al. 2010). However, methods have improved dramatically in recent years to the point where it is now possible to simultaneously track all members of entire groups of fish (Stienessen and Parrish 2013) or starlings (Ballerini et al. 2008; Cavagna et al. 2013) in three dimensions using fixed installations of cameras in a particular location. These methods are extremely useful for studying animal movement and decision processes, but the fixed instrumentation limits their ability to be applied in the field to follow a group of freely moving animals over longer intervals of space and time.

Over the years, researchers have taken various approaches to estimating positions of individual animals in the field. For example, Fischhoff et al. (2007) used periodic video censuses to record the relative position of zebras during single-file travel, and King et al. (2011) observed groups of baboons from a cliff top to visually estimate individual positions and timing during movement initiation. The marine environment provides particular challenges for tracking quickly moving individuals in a group. Animals often dive out of view, and they may surface asynchronously, unpredictably and often only for brief moments. Efforts to locate marine animals have often relied on estimating positions by eye from a distance with practicality requiring estimates to be grouped into broad spatial categories that are sampled at several minute intervals (Mattson et al. 2005). Calibrating visual observer estimates by periodically comparing them with a laser range 
finder or comparing estimates to a GPS buoy such as Visser et al. (2014) did when following groups of pilot whales from 100 to $400 \mathrm{~m}$, can improve accuracy and precision. However, in general, humans are often ineffective at estimating distance at sea by eye, subject to biases and large errors and with a tendency to lump measurements together (Buckland et al. 2001).

At sea, positions have been estimated by using the vertical angle between an object and the horizon (Gordon 2001), from overhead video cameras mounted on tethered airships (Nowacek et al. 2001), and by combining laser range finders with compass bearings measured with a GPS (Curé et al. 2012). Some approaches to monitoring marine animals from land can use theodolites, which measure the horizontal and vertical angles to a target (Bejder et al. 2006; Williams and Ashe 2007), but these instruments require a stable platform and they generally only sample one individual at a time. Thus, new equipment would be helpful for studying fast-moving groups of animals in the field over longer time periods and distances.

An ideal method would provide the ability to obtain a quickly updating 3D position of each animal in a group. This could be accomplished by instrumenting all the animals with biologging systems as has been done in pigeons (Nagy et al. 2010; Flack et al. 2013; Nagy et al. 2013). However, at present, few tags have the spatial and temporal resolution required for most behavioural analyses, and tagging all group members may not always be possible.

Here, we report on a portable stereo camera system that takes advantage of miniaturized digital technology to implement stereo photogrammetry for rapid and easy positioning of multiple animals. We demonstrate the performance of this system by 
simultaneously tracking the individual positions of six long-finned pilot whales,

Globicephala melas, allowing us to estimate inter-animal distances and group dispersion as a function of time with high spatial and temporal resolution.

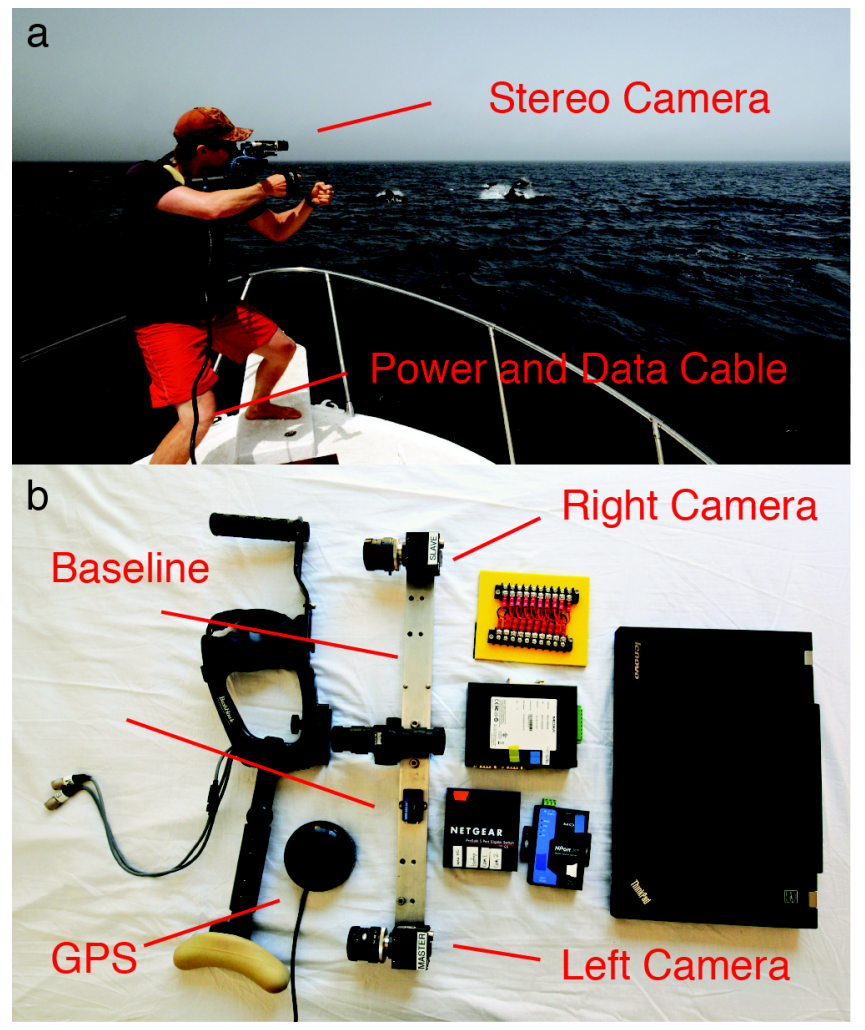

Figure 5.1: Stereo camera geocoding system. a System in action collecting data on a group of long-finned pilot whales, Globicephala melas. b System in parts shown without wiring and battery.

\subsection{SYSTEM DESCRIPTION}

\subsubsection{Overview of the system}

Our portable, battery-powered system (Fig. 5.1) uses online stereo photogrammetry to position animals in space and time. The system integrates a GPS receiver (GPS16x, Garmin, USA) to provide camera location, an attitude sensor (3DMGX3, Microstrain, USA) to identify the 3D direction in which the camera is aiming and a 
pair of computer-controlled calibrated and synchronized stereo digital cameras (Prosilica GC 1380, Allied Vision Technologies, Canada) to measure the range to any point in the stereo overlap area. The stereo camera is attached to an adjustable rigid baseline and sits on a modified wildlife photography shoulder mount (Bushhawk, USA). The centre of the stereo baseline has a sight for aiming. A $10-\mathrm{m}$ power and data cable connects to a $40-\mathrm{L}$ support cooler where a laptop running the Ubuntu 12.04 operating system and custom software written in $\mathrm{C}$ controls the data collection and processing. The entire system runs off a 12-V battery. The system's trigger is integrated into the photography mount, and three LEDs on the mount allow the operator to monitor whether data are being collected correctly. Often the operator can observe contextual details during data collection that are unavailable during analysis; therefore, a small headset microphone can be triggered to record time-stamped voice notes, such as animal IDs, that are saved as small audio files and synchronized with images from the camera. Although animals can often be identified from the captured images, these notes are a particularly useful complement with similar looking animals that may be difficult to distinguish, for example if they are facing away from the camera.

To operate the system, the operator sights on an animal or a group of animals and pulls the trigger in the camera mount. The LEDs signal that the system is recording, and stereo image pairs are captured at a predetermined rate of up to eight image pairs per second. The data from the GPS and attitude sensor are recorded into the metadata of each stereo pair. The voice recorder turns on whilst the trigger is pressed, and the system continues capturing data until the trigger is released. Battery life depends on the type of battery used as well as operating procedures: the current configuration of a small 32- 
Amp-hour car battery allows $6-8 \mathrm{~h}$ of data collection following a group of pilot whales and recording all individual surfacings.

\subsubsection{Technical approach to photogrammetric ranging}

Fig. 5.2 illustrates the basic principal concept of photogrammetric ranging (for a review, see Bradski and Kaehler (2008)).

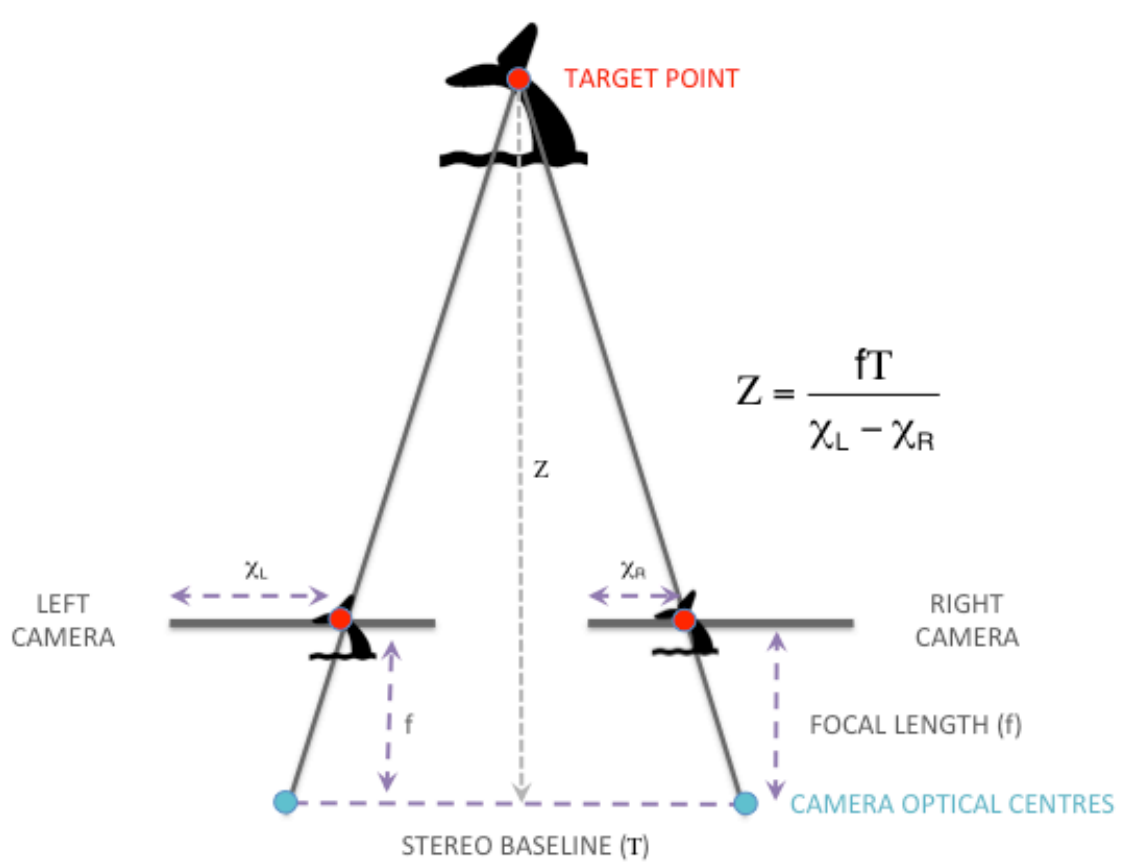

Figure 5.2: Technical approach to photogrammetric ranging. A conceptual illustration of photogrammetric ranging using a frontal parallel 2D view. Disparity between the corresponding images of the target in the two calibrated cameras, shown here by the difference between $\chi_{L}-\chi_{R}$ allows triangulation of the range in space $(Z)$ according to the following equation: $Z=\frac{f T}{\chi_{L}-\chi_{R}}$ where $f$ is the focal length of the two cameras and $T$ is the system baseline. 
A pair of calibrated cameras capture a stereo image of the target, in this case a whale fin. Lines are traced from the optical centre of each camera through the two points in the image plane corresponding to the same location on the target, and their intersection in the local camera reference frame is computed using triangulation. The system computes geodetic coordinates for the target using camera position and orientation data provided by the GPS receiver and the attitude sensor.

\subsubsection{System calibration}

In order to estimate range accurately, the system must take into account any distortion in the lenses and the relative geometry of the two cameras to each other in all six degrees of freedom (x, y, z, pitch, yaw and roll). Both of these factors were addressed using standard stereo calibration approaches found in the OpenCV computer vision software (Bradski and Kaehler 2008). OpenCV version 2.4 was used for all of the analyses described in this paper. The approach uses multiple stereo pairs of a checkerboard of known size and geometry to produce a set of calibration matrices that map the real-world system onto the idealized model illustrated in Fig. 5.2.

\subsubsection{Analytical method}

Data collection produces a directory of stereo image pairs similar to the example shown in Fig. 5.3a. 


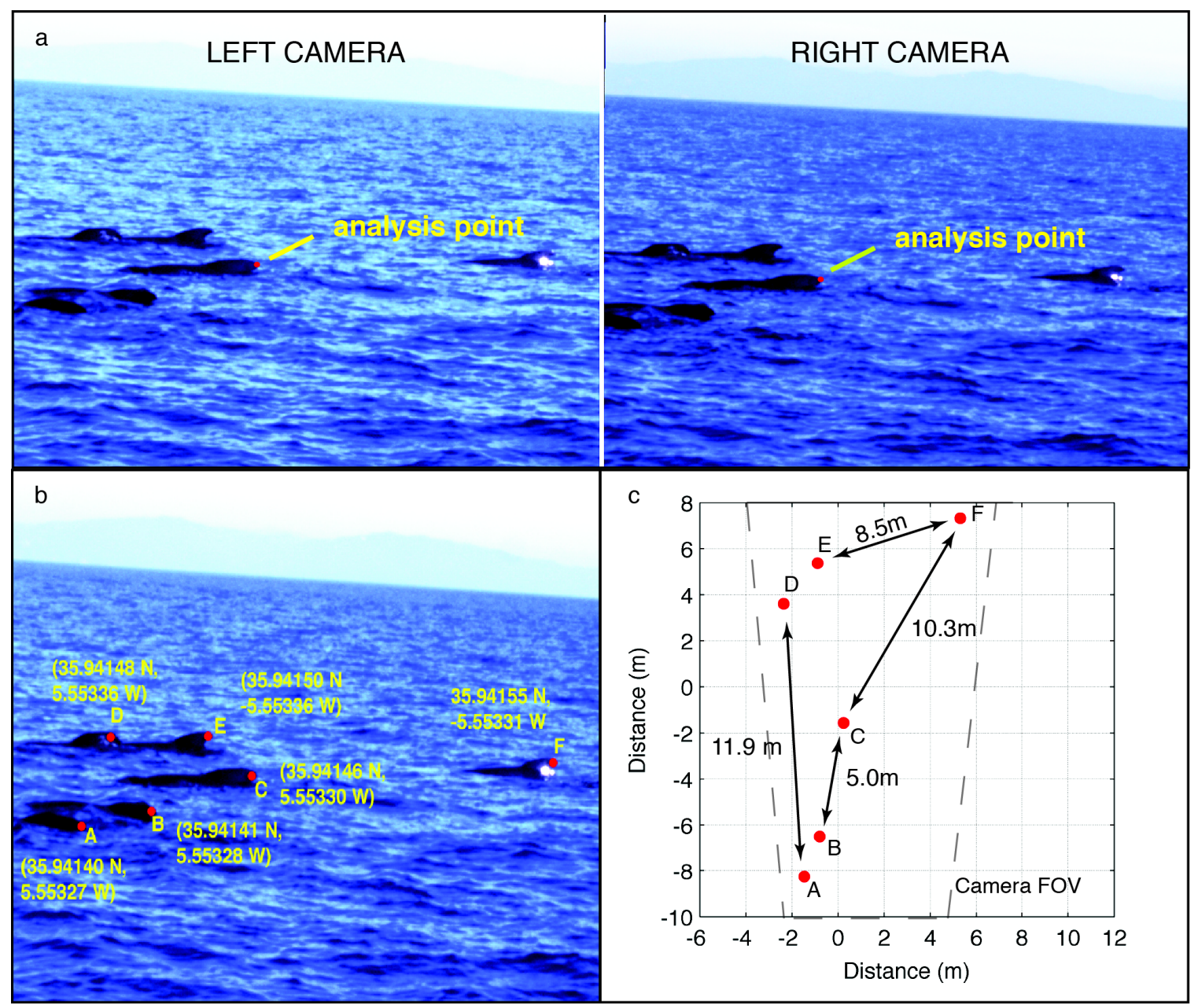

Figure 5.3: a Example stereo pair produced by the system from the left and right cameras. The corresponding positions of an analysis point chosen from simultaneous images in both cameras are shown for which the system can produce geodetic coordinates. b Left camera view of the stereo pair in a with the whales' geodetic coordinates. c Whales from b mapped onto a plan view. The system was located at (1.9, -35.5). Several examples of inter-animal distance are also shown. Notice how difficult it would be to reproduce an accurate representation of position or distance by eye alone.

Each pair is time-stamped and includes the metadata necessary for extracting positions using our custom software. When an image pair is loaded, the software uses the calibration parameters determined previously, performs an epipolar rectification on the pair and loads them into windows on the computer screen. Zooming in and out if necessary, the operator moves a cursor over a target in the left image (see the "analysis 
point" shown in Fig. 5.3a). The software then automatically cross-correlates the corresponding pixel in the right image using the Open CV matchTemplate function, a 32x32 window and the CV_TM_CCOR_NORMED match method. Should the software fail to automatically find the correct point in the right hand image, the operator can manually choose the point.

Using the coordinates of the target point in the epipolar lines, the software computes the coordinates of the point in the camera pair coordinate system. In essence, this is a vector from the camera pair to the target. The attitude measurements extracted from the metadata are used to compute a direction cosine matrix, which is used to multiply the target vector, producing a vector in a real-world (north-east-down, or NED) coordinate system whose origin is at the camera pair.

Since the GPS based camera location has been measured and preserved in the image metadata, it can be used to convert the NED target coordinates to geodetic coordinates. We use a scaled equirectangular projection, but given the very small geographic area over which we make measurements in NED frame, the details of the NED projection type do not contribute to accuracy considerations in any meaningful way (For pseudocode of the location reconstruction algorithm, please see Online Resource 1. The source code is available on request and can be obtained by emailing the authors).

Coordinates of target points from the stereo images and the integrated metadata are exported to an output file that can be easily opened in analysis programs such as MATLAB (Mathworks, Natick, USA). When a target point is recorded, the operator has the opportunity to apply a written label—for example, of the animal's ID stemming from the corresponding voice tag - that is stored with the output. Fig. 5.3b shows the left hand 
frame of the pair shown in 5.3a with the whales' geodetic latitude and longitude coordinates. Fig. 5.3c maps those positions into a plan view coordinate system looking down at the water's surface, demonstrating example inter-animal distances at that point in time.

\subsubsection{Accuracy considerations}

Limitations in the accuracy of this system stem primarily from errors in the GPS position, pointing vector from the attitude sensor and range estimated from stereo pair images. GPS errors are well documented, and there are established methods of filtering data for reducing them as much as possible depending on the particular receiver used (e.g., Hide, et al. 2003; Han and Wang 2012). Pointing errors in direction from the attitude sensor contribute to position error as a function of distance with greater range to target leading to larger errors. The specifications of the attitude sensor used are a rootmean-square (RMS) pointing error of $\pm 1^{\circ}$. A series of 107 measurements of a fixed sensor yielded RMS errors of $0.062^{\circ}, 0.035^{\circ}$ and $0.185^{\circ}$ for pitch, roll and heading. Camera pitch and roll mostly affect altitude errors, which are not a significant concern in most marine mammal research where the targets are on the ocean's surface; however, they could be more important if the system was used with terrestrial or airborne animals. Heading, on the other hand, is key for estimating the location of individual points. Heading error can be attributed to systematic deviation due to local magnetic fields and to inaccuracy in the heading estimate of the attitude sensor. We corrected for local geomagnetic offsets using the World Geomagnetic Model 2010; Iron errors caused by magnetic fields near the system require calibration in situ, but systematic deviation should not affect the relative location of sequential points. The attitude sensor was chosen 
for its high accuracy and update rate - the measured error of the heading estimate $\left(0.2^{\circ}\right.$ RMS) propagates into a static location error of $0.16 \mathrm{~m} \mathrm{RMS}$ at $50 \mathrm{~m}$, so this is negligible compared to the observed errors. Attitude measurements and image exposures occur asynchronously; the metadata for each image contains the most recent attitude measurements. This could cause time offsets between image exposures and attitude measurements, but the high-update rate of the attitude sensor (typically $20 \mathrm{~Hz}$ ) minimizes this error contribution under typical working dynamics.

Errors in stereo camera ranging stem from image errors caused by lens distortion and stereo pair-based errors. Image errors are addressed by calibration, but this will always have limits to its precision. Stereo pair-based errors result from choosing slightly different points in the left and right images leading to an incorrect disparity measurement. Because estimated range is proportional to baseline divided by disparity, the system's stereo baseline directly affects how this error is propagated through to the final measurement (Howland et al. 2012). A larger baseline reduces positioning error but also increases the minimum operating distance before a target is within the stereo overlap area. Thus, the choice of an appropriate stereo camera baseline will have to be based on considerations of typical range to target animals coupled with logistical and practical limitations on the size of equipment. We designed our system using an appropriate baseline for a target distance of up to approximately $50 \mathrm{~m}$ that we commonly use in focal follows. A larger baseline could easily extend the working distance.

To illustrate the range estimation accuracy of our system, we captured stereo pairs for targets located at 5-m intervals in a shore-based test using a 50-cm baseline and lenses with $25-\mathrm{mm}$ focal lengths. All measurements in this paper use this baseline and focal 
length. Fig. 5.4 shows errors gradually increasing with distance from the system, ranging from an average of $0.1 \mathrm{~m}$ at a $5-\mathrm{m}$ range to $3.2-\mathrm{m}$ error at a 50-m range. As with all stereo photogrammetric systems that rely on the disparity between two images in order to estimate distance, both accuracy and precision decrease with range. 

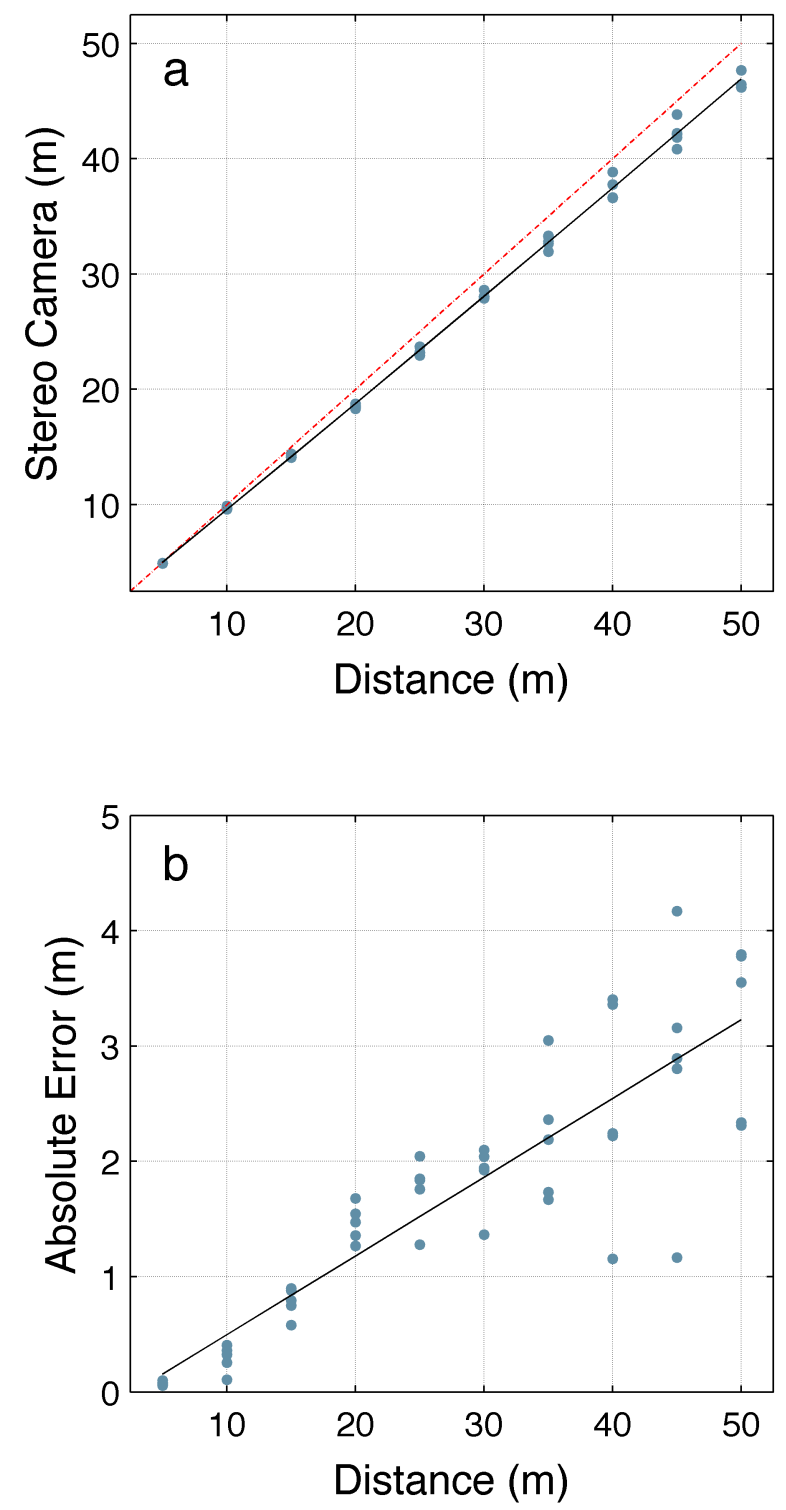

Figure 5.4: System accuracy at increasing range using a $50-\mathrm{cm}$ baseline and lenses with $25-\mathrm{mm}$ focal lengths. Five shore-based estimates were taken at 5-m intervals. a System's estimated range compared to actual range. The dotted red line represents a hypothetical perfect estimate. b Absolute error for each estimate at the intervals shown in a. The solid line is a linear best fit through the error scores. Average deviation ranged from $0.1 \mathrm{~m}$ at 5 to $3.2 \mathrm{~m}$ at a $50-\mathrm{m}$ range. As with all stereo photogrammetric systems that rely on the disparity between two images in order to estimate distance, both accuracy and precision decrease with range 
Figure 5.5: System accuracy at increasing range measured on the water in a calm sea state (Beaufort 2). a Absolute error of a distance measurement between 2 traffic cones placed $3 \mathrm{~m}$ apart (a relevant inter-animal distance) on shore at increasing ranges with the system deployed from a small unstable vessel. Assessing accuracy of range with the system at sea is challenging, for even a small anchored vessel will move about, and the measurement tool used will have its own error curves. Range for comparison with the system was measured with a range finder, but should be understood to include a range of values of $+/-3$ to $4 \mathrm{~m}$ to account for boat movement. Distances were taken at opportunistic intervals when it was possible to stabilize the vessel as much as possible against moorings. The solid line is a linear best fit through the error scores. The average absolute value of the error ranged from $0.15 \mathrm{~m}$ at $\sim 12 \mathrm{~m}$ up to $1.69 \mathrm{~m}$ at $\sim 63 \mathrm{~m}$. To get a conservative estimate of geodetic location reconstruction accuracy, $\mathbf{b}$ shows absolute error of a geodetic coordinate calculated using the system compared with ones measured by placing the GPS receiver on top of the target. Like a, the positions were calculated at increasing distances from a target on shore. In addition to incorporating all measurement and location reconstruction error, this plot includes both error in the system GPS and again in the GPS measurement of the target's position without the system, leading to a conservative estimate. The solid line is a linear best fit through the error scores. Average absolute error ranged from $2.5 \mathrm{~m}$ at $\sim 9 \mathrm{~m}$ up to $25 \mathrm{~m}$ at $\sim 63 \mathrm{~m}$, which is beyond the design range of a system with this baseline and focal length 

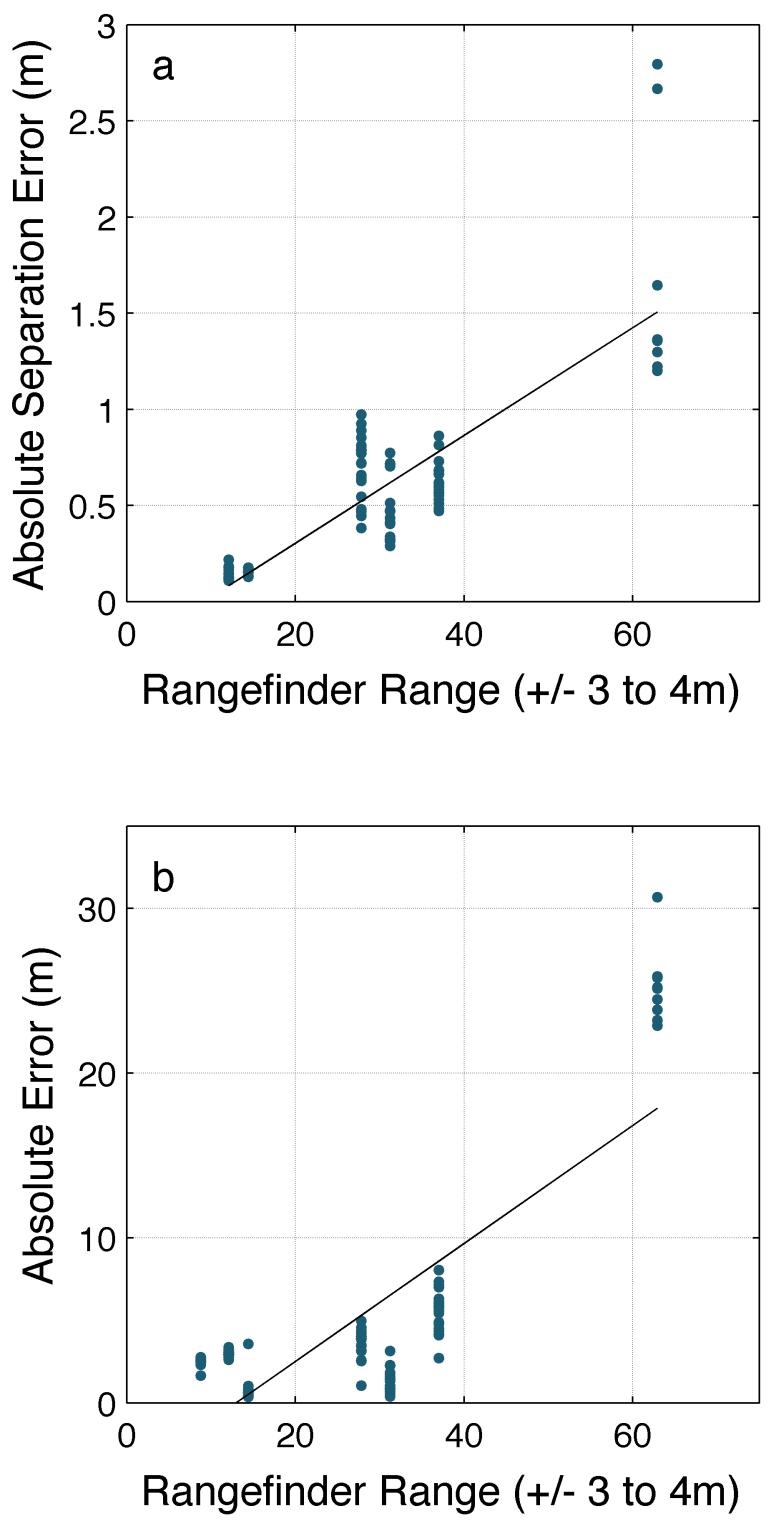
When collecting data at sea in varying conditions, platform instability can be a concern. We designed our system with this in mind, using fast shutter speeds and an attitude sensor with a high update rate to mitigate any potential effects of movement resulting from sea state. With respect to the attitude sensor, it is not possible to completely separate gravitational acceleration from body acceleration, such as that caused by vessel motion without using a GPS-based attitude system that would be too bulky for our application; however, the sensor's frequency-based filtering and combination of accelerometer and magnetic measurements help minimize this error. Given this limited effect of platform instability, we used shore-based tests where it is possible to carefully measure and control all aspects of a trial to provide the most appropriate estimate of the system's accuracy. However, to give a sense of how accuracy might change in an unstable platform, we also conducted measurement trials from a floating vessel that show similar results (Fig. 5.5).

It is important to remember that aside from the system's inherent accuracy, there are certainly other difficulties arising from operating in heavy seas, such as effects on the ability of those conducting the focal follow to easily see the animals; however, these would be a limitation when using almost any kind of observation technique.

\subsection{FIELD TEST AND EXAMPLE DATA}

We field-tested the system on a 15-min focal follow of a group of travelling longfinned pilot whales (G. melas) travelling in the Strait of Gibraltar. Sea state conditions were Beaufort 3-4. The group consisted of six individually identified pilot whales, a large adult male, a female, her calf and three young adult animals of indeterminate sex. Using the stereo camera system, the position of each animal was recorded every time it 
surfaced, 336 measurements in total. We used the tip of the dorsal fin to define animal position because this was the most consistently visible point. Animals were diving intermittently and did not necessarily surface at the same time, so a 2D linear interpolation was used to estimate the 2D surface position of each animal between surfacings (i.e., if the animal's position at depth was $(\mathrm{x}, \mathrm{y}, \mathrm{z})$, the projection on the surface was $(\mathrm{x}, \mathrm{y}, 0)$. Inter-animal distances were then estimated from the interpolated surface positions of each individual. Finally, a measure of overall group dispersion was estimated as the RMS Euclidian distance between the interpolated surface position of each animal and the group centroid position. The system provides animal positions in time at each surfacing, and there are many potential ways of creating tracks beyond the linear interpolation we have used to illustrate this example.

Fig. 5.6a shows estimated animal tracks from these positions over a $15-\mathrm{min}$ period. Tracks are shown in Northing and Easting with the initial position of the first animal as the origin. Although the animals stayed within a $150 \times 250-\mathrm{m}$ area, they were actually swimming steadily the entire time against a strong current. The circle, square, diamond and triangle markers super-imposed on the tracks represent individual animal positions at 3-min intervals. 
Figure 5.6: a Interpolated tracks of animal positions of a group of six known long-finned pilot whales over 15 min. Tracks are calculated from 336 measured positions across the 6 animals. Tracks are shown in Northing and Easting with respect to the initial position of the first animal sampled. Although the animals do not appear to have travelled far, they were swimming the entire time against a strong current. The circle, square, diamond and triangle markers represent identical points in time. b Dashed black line shows estimated inter-animal distance (in meters) between the mother and calf in Fig. a, calculated from 72 positions for the mother and 69 for the calf. Blue solid line shows surface cohesion of the entire group over time. Group dispersion was described as instantaneous group dispersion, defined as the RMS distance of individual positions relative to the group centroid at that instant in time. Note that this metric varies as a function of the number of individuals being tracked. Dispersion and distance were calculated using the 2D linear interpolated tracks from a 

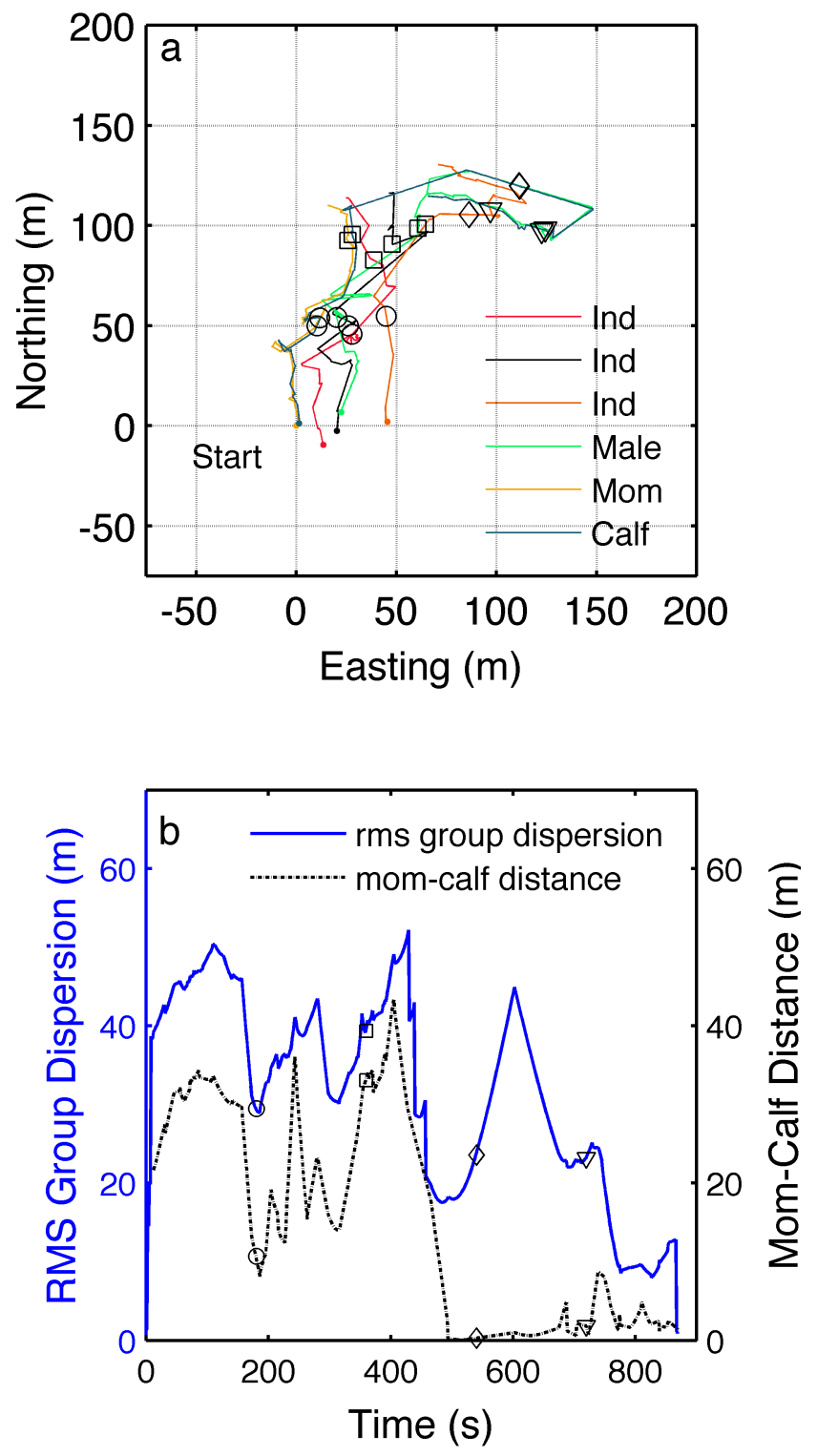
We were then able to use these interpolated positions to estimate inter-animal distance over time; for example, Fig. 5.6b shows the distance between the mother and her calf. We described group dispersion as the RMS distance of each group member's position to the group's centroid at that instant in time. Note that this particular metric is linked to the number of individuals being tracked. Fig. 5.6b also shows estimated group dispersion over time. A higher value of dispersion indicates an increase in spread. It was calculated using the interpolated tracks from Fig. 5.6a. Most animals were surfacing several times a minute, but those that were in the midst of a dive lasting 5 min or longer were excluded from the analysis in order to reduce error from linear interpolations. The same time markers used in Fig. 5.6a appear on Fig. 5.6b. A 15x speed video of overhead geodetic position with a running calculation of RMS dispersion is shown in Online Resource 2.

\subsection{DISCUSSION}

We have developed a 3D stereo geocoding system that facilitates tracking multiple animals in space and time. The system is especially useful in field situations where it is necessary to track multiple animals within a group over relatively long distances and time periods whilst positioning individuals with high spatial and temporal resolution. The data collected can be used to calculate a variety of metrics beyond animal position; in particular, they can be used to estimate group dispersion. The system can also be easily combined with other methods such as biologging tags.

The system provides a combination of features useful for studying animals in the

field, particularly in situations that require simultaneous high spatial and temporal 
resolution tracking of multiple individuals during a follow. First, it is portable and can be deployed from a small vehicle or research vessel. Second, it is easily aimed in any direction and does not require vessel manoeuvring to position animals in a fixed field of view. Third, it provides high spatial and temporal resolution positioning of multiple group members. As the apparent differences between Fig. 5.3b and 5.3c illustrate, estimating static positions and inter-animal distances by eye or from a fixed image is hard to do with high accuracy. Even trained visual observers are likely to judge distance in the foreshortened axis differently from the perpendicular left-right axis. Our system removes that uncertainty by providing quantitative, repeatable measurements regardless of the operator. Furthermore, whilst practicality often restricts behavioural observation protocols relying on human observers to a several minute sampling interval, the $8-\mathrm{Hz}$ temporal resolution of this system permits the study of finer-scale behavioural changes than may be possible to measure with observers alone. Both the spatial and temporal resolution depend on the particular configuration used, and the system is completely adjustable with baseline, cameras, lenses, sample rate, shutter speed and aperture, all changeable based on the particular needs of a study. Finally, it allows multiple animals to be sampled simultaneously if captured in the same frame or in quick succession if not.

The positions captured by the system can generate other behavioural metrics beyond the inter-animal distance illustrated in Fig. 5.6b. For example, different positions in time could be used to calculate a measure of speed over ground. Likewise, the georeferenced images would allow heading to be calculated to measure any deviations that might result from environmental factors such as a disturbance (Curé et al. 2012). Even the decision about whether marine animals are associated in the same group is usually 
based on a definition that uses inter-animal distance as a discrimination parameter (Whitehead and Dufault 1999; Whitehead et al. 2000), so the temporal and spatial resolution of the system could aid assessment of group size and social structure.

In particular, the ability to estimate changes in surface group dispersion over time will be useful for monitoring social behaviour, responses to predators and the effects of disturbance. Many animals adopt social defence strategies to predators, relying on their group members to protect them (Hamilton 1971; Krause and Ruxton 2002). In the face of a threat, animals using a social defence strategy may increase social group cohesion as measured by reduced inter-animal distance (Bode et al. 2010). Thus, dispersion is an important proxy for disturbance or stress in many species, both in connection with natural predators (Altmann 1956; Macdonald 1983; Pitman et al. 2006) as well as anthropogenic stressors such as ships and noise (Nowacek et al. 2001; Bejder et al. 2006). The continuous fine-scale estimates of dispersion that the system permits (see Fig. 5.6b and Online Resource 2) will aid characterization of spatio-temporal behavioural changes, be they the result of a controlled disturbance or other recorded cues.

The system can be used in tandem with a variety of other methods and behavioural data. For example, in situations where animals cannot be viewed continuously, researchers often rely on individual markings to determine individual identity. These determinations are easily recorded with the integrated vocal labelling to provide continuous tracking. Moreover, although a feature of the system is that it does not require instrumenting animals, it can be combined with biologging methods such as archival acoustic and kinematic tags (eg., Johnson and Tyack, 2003). In marine mammals, instrumenting animals with tags would allow the integration of surface data 
(e.g., the 2D illustration shown in Fig. 5.6a, b) with underwater tracks to estimate 3D positions above and below the surface, in turn extending cohesion calculations and other metrics to three dimensions. Furthermore, when acoustic tags are able to identify calls from individuals or groups, the combination with the system would enable investigation of acoustic cues that might mediate any number of the fine-scale behavioural metrics the system can observe. The continuous tracking of animal location from the system could also help provide animal position with respect to other geo-referenced environmental parameters such as topographical features, a tagged predator, an acoustic array for localizing signals or anthropogenic disturbances.

There are many good techniques for positioning animals in space and time, and some field biologists will prefer pen and paper to a system like this whose resolution comes at the cost of purchasing and maintaining equipment and extra analysis time. We have developed our system for the field situation where multiple animals must be sampled at high spatial and temporal resolution whilst moving too far during the follow period to be tracked with fixed equipment installations. The system samples up to eight times a second and allows simultaneous localization of multiple animals to distances of $50-100 \mathrm{~m}$ or more depending on the configuration and resolution required. These positions provide quantitative estimates of a variety of other metrics such as inter-animal distances, group dispersion, speed and heading. This system is particularly useful in combination with other techniques such as archival tags, photo-identification strategies or sound playback experiments to facilitate fieldwork investigating topics ranging from natural animal behaviour to how animals respond to anthropogenic disturbances. 


\subsection{ACKNOWLEDGEMENTS}

This work could not have been completed without valuable contributions from many different people. We are grateful to Baxter Hutchinson and Alessandro Bocconcelli for help with the fabrication and logistics. Philippe Verborgh, Pauline Gauffier, Renaud de Stephanis and the Center for Investigation and Research on Cetaceans (CIRCE) were essential for the fieldwork evaluating the system. This paper also benefitted from useful suggestions by two anonymous reviewers. Research was funded in part by the Office of Naval Research (grants N000140910528 and N000141210417) and the Woods Hole Oceanographic Institution Marine Mammal Center. FHJ was supported by the Danish Council for Independent Research | Natural Sciences and is currently funded by the Carlsberg Foundation. PLT was supported by the Scottish Funding Council (grant HR09011) through the Marine Alliance for Science and Technology for Scotland. 


\subsection{ETHICAL STANDARDS}

The experiments comply with Spanish and United States laws and were approved by the WHOI Institutional Animal Care and Use Committee. Fieldwork was conducted under United States National Marine Fisheries Service permit \#14241 to PLT.

\subsection{CONFLICT OF INTEREST}

The authors have no financial relationship with the sponsors. 


\subsection{REFERENCES}

Altmann, S. A. (1956). Avian mobbing behavior and predator recognition. The Condor, 58(4), 241-253.

Ballerini, M., Cabibbo, N., Candelier, R., Cavagna, A., Cisbani, E., Giardina, I., . . . Viale, M. (2008). Empirical investigation of starling flocks: a benchmark study in collective animal behaviour. Animal Behaviour, 76(1), 201-215.

Bejder, L., Samuels, A., Whitehead, H., \& Gales, N. (2006). Interpreting short-term behavioural responses to disturbance within a longitudinal perspective. Animal Behaviour, 72(5), 1149-1158.

Bode, N. W., Faria, J. J., Franks, D. W., Krause, J., \& Wood, A. J. (2010). How perceived threat increases synchronization in collectively moving animal groups. Proceedings of the Royal Society B: Biological Sciences, 277(1697), 3065-3070.

Bode, N. W., Wood, A. J., \& Franks, D. W. (2011). The impact of social networks on animal collective motion. Animal Behaviour, 82(1), 29-38.

Bradski, G., \& Kaehler, A. (2008). Learning OpenCV: Computer vision with the OpenCV library: O'Reilly Media, Inc.

Buckland, S. T., Anderson, D. R., Burnham, K. P., Laake, J. L., Borchers, D. L., \& Thomas, L. (2001). Introduction to distance sampling: estimating abundance of biological populations. Oxford: Oxford University Press.

Cavagna, A., Queirós, S. D., Giardina, I., Stefanini, F., \& Viale, M. (2013). Diffusion of individual birds in starling flocks. Proceedings of the Royal Society B: Biological Sciences, 280(1756), 20122484.

Conradt, L., \& Roper, T. (2000). Activity synchrony and social cohesion: a fission-fusion model. Proceedings of the Royal Society B: Biological Sciences, 267(1458), 2213-2218.

Conradt, L., \& Roper, T. J. (2003). Group decision-making in animals. Nature, 421(6919), 155-158.

Conradt, L., \& Roper, T. J. (2010). Deciding group movements: Where and when to go. Behavioural Processes, 84(3), 675-677.

Couzin, I. D., Krause, J., Franks, N. R., \& Levin, S. A. (2005). Effective leadership and decision-making in animal groups on the move. Nature, 433(7025), 513-516.

Curé, C., Antunes, R., Samarra, F., Alves, A. C., Visser, F., Kvadsheim, P. H., \& Miller, P. J. (2012). Pilot whales attracted to killer whale sounds: acoustically-mediated interspecific interactions in Cetaceans. PLoS ONE, 7(12), e52201.

Da Cunha, R. G. T., \& Byrne, R. W. (2009). The use of vocal communication in keeping the spatial cohesion of groups: Intentionality and specific functions. In P. A. Garber, A. Estrada, J. C. Bicca-Marques, E. W. Heymann \& K. B. Strier (Eds.), South American Primates (pp. 341-363): Springer New York.

Fischhoff, I. R., Sundaresan, S. R., Cordingley, J., Larkin, H. M., Sellier, M.-J., \& Rubenstein, D. I. (2007). Social relationships and reproductive state influence leadership roles in movements of plains zebra, Equus burchelli. Animal Behaviour, 73(5), 825-831.

Flack, A., Freeman, R., Guilford, T., \& Biro, D. (2013). Pairs of pigeons act as behavioural units during route learning and co-navigational leadership conflicts. The Journal of experimental biology, 216(8), 1434-1438. 
Gordon, J. (2001). Measuring the range to animals at sea from boats using photographic and video images. Journal of Applied Ecology, 38(4), 879-887.

Gueron, S., \& Levin, S. A. (1993). Self-organization of front patterns in large wildebeest herds. Journal of Theoretical Biology, 165(4), 541-552.

Hamilton, W. D. (1971). Geometry for the selfish herd. Journal of Theoretical Biology, 31(2), 295-311.

Han, S., \& Wang, J. (2012). Integrated GPS/INS navigation system with dual-rate Kalman Filter. GPS solutions, 16(3), 389-404.

Hide, C., Moore, T., \& Smith, M. (2003). Adaptive Kalman Filtering for Low-cost INS/GPS. The Journal of Navigation, 56(01), 143-152. doi: doi:10.1017/S0373463302002151

Howland, J. C., Macfarlane, N., \& Tyack, P. (2012). Precise geopositioning of marine mammals using stereo photogrammetry. Paper presented at Oceans, 2012, Hampton Roads.

Johnson, M. P., \& Tyack, P. L. (2003). A digital acoustic recording tag for measuring the response of wild marine mammals to sound. IEEE Journal of Oceanic Engineering, 28(1), 3-12.

King, A. J., Sueur, C., Huchard, E., \& Cowlishaw, G. (2011). A rule-of-thumb based on social affiliation explains collective movements in desert baboons. Animal Behaviour, 82(6), 1337-1345.

Krause, J., \& Ruxton, G. D. (2002). Living in groups: Oxford University Press.

Macdonald, D. W. (1983). The ecology of carnivore social behaviour. Nature, 301(5899), 379-384.

Mattson, M. C., Thomas, J. A., \& Aubin, D. S. (2005). Effects of boat activity on the behavior of bottlenose dolphins(Tursiops truncatus) in waters surrounding Hilton Head Island, South Carolina. Aquatic Mammals, 31(1), 133-140.

Nagy, M., Ákos, Z., Biro, D., \& Vicsek, T. (2010). Hierarchical group dynamics in pigeon flocks. Nature, 464(7290), 890-893.

Nagy, M., Vásárhelyi, G., Pettit, B., Roberts-Mariani, I., Vicsek, T., \& Biro, D. (2013). Context-dependent hierarchies in pigeons. Proceedings of the National Academy of Sciences, 110(32), 13049-13054.

Nowacek, S. M., Wells, R. S., \& Solow, A. R. (2001). Short-term effects of boat traffic on bottlenose dolphins, Tursiops truncatus, in Sarasota bay, Florida. Marine Mammal Science, 17(4), 673-688.

Parrish, J. K., \& Hamner, W. M. (1997). Animal groups in three dimensions: how species aggregate: Cambridge University Press.

Pitman, R. L., Ballance, L. T., Mesnick, S. I., \& Chivers, S. J. (2006). Killer whale predation on sperm whales: observations and implications. Marine Mammal Science, 17(3), 494-507.

Stienessen, S. C., \& Parrish, J. K. (2013). The effect of disparate information on individual fish movements and emergent group behavior. Behavioral Ecology, 24(5), 1150-1160.

Sumpter, D., Buhl, J., Biro, D., \& Couzin, I. (2008). Information transfer in moving animal groups. Theory in biosciences, 127(2), 177-186.

Visser, F., Miller, P. J., Antunes, R. N., Oudejans, M. G., Mackenzie, M. L., Aoki Kagari, A., ... Tyack, P. L. (2014). The social context of individual foraging 
behaviour in long-finned pilot whales (Globicephala melas). Behaviour, 151(10), 1453-1477.

Whitehead, H., Christal, J., \& Tyack, P. L. (2000). Studying cetacean social structure in space and time. In J. Mann, R. Connor, P. Tyack \& H. Whitehead (Eds.), Cetacean societies: field studies of dolphins and whales (pp. 65-86). Chicago: University of Chicago Press.

Whitehead, H., \& Dufault, S. (1999). Techniques for analyzing vertebrate social structure using identified individuals: review and recommendations. Advances in the Study of Behavior, 28, 33-74.

Williams, R., \& Ashe, E. (2007). Killer whale evasive tactics vary with boat number. Journal of Zoology, 272(4), 390-397. 
Chapter 6: Conclusion 


\subsection{FRAMING REMARKS}

Bottlenose dolphins are intensely social animals who live in fission-fusion societies, forming multiple brief associations amid strong stable relationships with a few key individuals (Connor et al., 2000; Mann et al., 2000). To navigate these complex relationships in a three-dimensional environment, they need mechanisms to stay in contact and maintain spatial cohesion over time. Touch and vision lack the range, and chemicals diffuse too slowly underwater, so the primary channel of communication will be acoustic (Da Cunha \& Byrne, 2009; Janik, 2009). This thesis explores these acoustic mechanisms in the wild. A critical element for parsing the functions of acoustic behavior is knowing the identity of a caller on an individual level. With groups of delphinids that can number in the thousands (Connor et al., 1998), this information is extremely challenging to obtain using surface observation methods, leading many wild studies to record from groups of animals rather than individuals (eg. Smolker et al., 1993; Cook et al., 2004). To resolve these problems, this thesis cannot rely solely on traditional observation methods; rather, it simplified the situation to study dyadic social relationships, focusing on pairs of closely-associated mothers and calves, and allied males.

This thesis describes the first successful deployments of on-animal acoustic tags on wild bottlenose dolphins, using simultaneously-tagged pairs of closely-associated animals to identify the calling individual with a high degree of confidence. It took advantage of the unique opportunity to deploy on-animal sound and movement recording tags during the Sarasota Dolphin Health Assessments, in order to characterize the temporal patterns of vocal behavior, investigate the function of signature whistles during 
separations and reunions, and assess whether dolphins keep track of their partners by monitoring conspecific echolocation clicks. Then it turned to the problem of studying cohesion without the possibility of deploying tags and reported the development of a novel stereo camera system for positioning both tagged and non-tagged animals in space and time.

\subsection{CHAPTER SYNOPSES}

\subsubsection{Chapter Two: Temporal patterns in bottlenose dolphin vocal behavior}

This chapter investigated the timing of different types of calls. By focusing on 115.01h of data from 12 simultaneously tagged dolphins, I was able to examine the temporal dynamics of acoustic behavior with a level of confidence that was not

previously possible for wild bottlenose dolphins. I first reported the rates of four different types of calls: signature and non-signature whistles, chirps and buzzes, and found a general trend of calves producing more whistles than mothers and males. Overall, vocal rates and calling behavior were quite variable between individuals, highlighting the importance of using on-animal tags that can attribute caller identity, and emphasizing the need to increase tag attachment time and sampling duration for future studies.

I then examined the shape of the distribution of inter-call-intervals using waiting time analysis to investigate the clustering of different call types. To address inter-animal variability, I looked within individuals and found a wide spread of waiting times between calls. Waiting time curves were significantly different, with each type of call having its own characteristic timing distribution for each animal. For example, 10 out of 11 animals had signature whistles that were much more clustered than non-signature whistles. 
Differences between the demographic groups of mothers, calves and males were only found for non-signature whistles, not signature whistles, buzzes or chirps. In combination with the observation of individual animals using a variety of non-signature whistles, and occasionally repeating non-signature whistles in bouts, this may suggest that the nonsignature whistle call type actually encompasses several subcategories. In general, different types of calls had significantly different timing distributions, suggesting that they may have different functional uses.

Looking specifically into signature whistles, I found that an animal's signature whistle increased the probability that its partner would respond with its own signature whistle. Furthermore, timing differences in response latency during these exchanges may reflect asymmetries in the relationships - two of the five pairs analyzed showed significant differences in response latency, and another mother-calf pair showed a strong trend $(p=0.056)$. Finally, I explored the heterogeneity in the probability of detecting a signature whistle, to evaluate a key assumption of many simple mark-recapture methods - that the probability of detection is the same across animals. Likelihood curves for the number of animals that could produce a given number of detected signature whistles suggest that this variability may prove challenging for that assumption, should those methods be applied to bottlenose dolphins.

6.2.2 Chapter Three: A reunion calling function for signature whistles in wild bottlenose dolphins

Chapter Three used the ability of simultaneous tagging to identify the vocalizing individual to study how pairs of bonded dolphins use signature whistles, a particularly well-studied type of call (e.g. Janik \& Sayigh, 2013). To investigate the cohesion functions of signature whistles, I focused on mother and calf pairs that are highly 
motivated to remain cohesive: young calves are extremely dependent on their mothers and vulnerable to shark attacks when separated (Heithaus, 2001), and these pairs have the highest coefficient of association of any group (Connor et al., 2000). Signature whistles have been linked with affiliation and social cohesion (Smolker et al., 1993; Janik \& Slater, 1998; Watwood et al., 2005), but the inability of scientists to identify the vocalizing individual or position animals in space and time has impaired tests of specific cohesion functions in wild animals (Watwood et al., 2005). Here, I investigated signature whistle use during separations (inter-animal distance increasing from $7 \mathrm{~m}$ out to a maximum that must be greater than $30 \mathrm{~m}$ ) and reunions (inter-animal distance decreasing from maximum separation back to $7 \mathrm{~m}$ ) events. These temporary separations reflect periods when animals are separated beyond the visual range, and where acoustic signals would be especially helpful to mediate relocation of conspecifics.

Modeling signature whistle use during these events allowed me to test three of their potential functions: 1) a location monitoring function where animals emit calls spontaneously during temporary separations and reunions in order to keep track of each other; 2) a reunion call function where signature whistles indicate a motivation to reunite with the partner; and 3) an identity advertisement function where individuals use signature whistles to confirm identity immediately prior to reuniting.

I found significantly higher signature whistle rates when an animal was in a reunion than a separation, an increase in probability of signature whistles with distance, and concentrations shortly after peak separation and immediately prior to reuniting. Taken together, these lines of evidence are consistent with a dual function of signature 
whistles used both for initiating reunions and for confirming the identity of conspecifics prior to rejoining each other at the end of a reunion.

\subsubsection{Chapter Four: Do dolphins keep track of their partners by passively monitoring conspecific biosonar signals?}

If dolphins can use signature whistles as a mechanism for mediating cohesion, what about the separations and reunions that do not include whistles? Like other toothed whales, dolphins gather information about their environment from echolocation, and these sonar clicks are audible to other animals. Chapter Four explored whether they could use these echolocation signals from conspecifics as another mechanism to keep track of partners when separated. A three-pronged approach investigated the range of a potential echolocation monitoring strategy by 1) modeling the distribution of maximum detected ranges of echolocation clicks from each dolphin; 2) separating clicks according to the angle of the beam pattern of the transmitting dolphin with respect to the receiver to create probability density functions of maximum detection; and 3) characterizing the effects of range in a separation and reunion on the probability of a click being detected. These lines of inquiry demonstrated that, despite individual needs to forage (Mann et al., 2000), typical separation distances of mother-calf pairs overlap with the operating range of an echolocation monitoring strategy. Moreover, incorporating echolocation monitoring into the model from Chapter Three helped explain the probability of an animal producing a signature whistle, suggesting that passively listening to a conspecific's biosonar clicks may allow a dolphin to monitor the location of a partner. 
6.2.4 Chapter Five: A 3D stereo camera system for precisely positioning animals in space and time

Chapters two, three and four have described simultaneous tagging methods for studying cohesion and communication, but there will be many more situations where we cannot, or do not want to, tag the animals. To study cohesion under those circumstances, we still need to know where the animals are. Therefore, Chapter Five described the development of a portable stereo camera system, integrating a GPS receiver, an attitude sensor and 3D stereo photogrammetry, to rapidly estimate the position of multiple animals in space and time. Chapter Five also describes how I tested the system and used it to measure cohesion on long-finned pilot whales, Globicephala melas, in the Strait of Gibraltar. This system can ground behavioral observations in quantitative metrics rather than relying on error-prone human vision to estimate distance, characterize fine-scale behavior, and detect changes as a result of a disturbance that might otherwise be difficult to observe.

\subsection{GENERAL OBSERVATIONS}

The explorations described in this thesis lead to two general observations on the study of cohesion and communication in social delphinids: we need to incorporate individual variability into models of communication and cohesion, and there are advantages to studying them in their natural habitat.

One of the recurring themes from these chapters has been the large amount of individual variability observed. Individual animals showed very different rates and timing distributions between different types of calls. Even when normalized by base rate, significant contrasts in waiting time distributions were obscured when averaged across 
animals. Just as differences were lost in averaging, so a data collection method that records across an entire group will be unable to accurately characterize biologically relevant differences between individual animals. This variability highlights the importance of on-animal tags for studying vocal behavior. However, even a single onanimal tag cannot effectively resolve this critical problem. Rather, the combination of a simplified study system with small groups of known animals, simultaneous tagging and behavioral observations monitoring for the presence of other animals in the area, forms the best approach to attribute caller identity. The addition of a long-term study of identified individuals contributes essential information to the observations, grounding the individual variability in the context of known relationships. This perspective is crucial for accurately interpreting cohesion and communication behaviors that will be heavily influenced by affiliation.

Along with acknowledging the individual variability, effectively exploring communication and cohesion will require studies on wild animals in addition to captive ones. Captive experiments can provide easier control and essential background, but dolphins evolved their behaviors for their natural environment, and it is difficult to assess how accurately captive communication and cohesion studies extrapolate to a dynamic and heavily context-dependent natural environment where animals can freely separate and select their associates. In an example of the difference between captivity and the wild, the click source levels estimated during separations and reunions (Chapter Four) were significantly lower than those reported from captive studies in the literature and more similar to wild values (Wahlberg et al., 2011). 
The combination of captive and wild studies is particularly powerful, and both are likely required for studying cohesion mechanisms such as signature whistle use. The simultaneous tagging methods described here have yet to be applied in captive settings, and their primary usefulness comes from the wild where other methods of recording and calculating distance are less readily available. However, in this thesis, I was able to use the simultaneous tagging methods to investigate the context of signature whistles because five decades of captive research had shown that dolphins had special signature whistles that appeared to be linked to cohesion. Without this captive research to provide the relevant background information, the focus of my work with wild animals would not have been possible. This lack of captive context presents a challenge for studying cohesion calls in other species of delphinids, such as pilot whales. They may have some type of signature whistle-like signal, but without long captive studies of their acoustic repertoire, it may be challenging to isolate in the wild with confidence.

Studies on wild bottlenose dolphins are challenging, particularly when scientists must track the behavior of individuals. However, this thesis demonstrates the possibilities that stem from working with on-animal tags and small groups of known individuals. For situations where tagging is not possible, tools like the stereo camera system can help, and they could also be combined with tag deployments, providing geodetic spatial information to position the underwater tracks from Chapter Three in absolute space.

\subsection{1: Some observations on the rate-limiting steps of stereo photogrammetry and the potential for using drones to collect similar data}

The rate-limiting steps in the analysis of the coordinate positions collected by the stereo-camera described in Chapter Five mostly stem from the time required to identify the target positions in the stereo pair of images. At the moment this must often be done 
manually, particularly when the colors of the dorsal fins do not provide a strong visual contrast with the water (such as is the case with bottlenose dolphins in high glare). Picking the points can also be more challenging at ranges when the resolution of the cameras causes the dorsal fins to become more pixelated. These limitations could be improved by more expensive higher-resolution cameras and by relatively straightforward computer vision processing to streamline the workflow of the analysis software and choose target points automatically.

Using drones, such as hexacopters, may provide an exciting alternative platform to collect spatial data. An advantage of drones is that they would be able to fly right above the animals without needing the close focal follows that the stereo camera requires. However, anecdotal evidence suggests that animals sometimes spy hop to observe the drones (Gauffier, P. P. Comm, Sept 10, 2014), and calibrated measurements of underwater noise would be essential to assess the acoustic stimulus produced by drones. The drones would have to be of a size that could be transported on a small vessel and would need to hold high enough resolution cameras to capture accurate spatial measurements. The biggest limitation of drones at the moment is probably battery life, particularly in high winds where they use more power to maintain stability. Battery life is on the order of minutes, and I've twice observed them crash into the water when they ran out of power. In contrast, the hand-held stereo camera can collect data for hours without stopping.

A particularly exciting possibility would be to have drones carry a similar calibrated pair of cameras like the stereocamera system in order to combine remote measurements from the air with 3D mensuration and accurate positions. The visual 
analysis challenges for the stereo camera system would be duplicated in a drone, but again automated visual processing could accelerate the workflow.

\subsection{FUTURE DIRECTIONS}

Building on the work described in this thesis, some of the most exciting opportunities for future research stem from greater sampling, either through longer deployments or a larger number of tagged animals, playback experiments to free-ranging individuals, and from combining the photogrammetry and tag methods together to begin working with larger groups of animals. It is only in the last four years that it has been possible to deploy acoustic tags on bottlenose dolphins, and although the 86 hours of simultaneous tag time provide an amazing initial window into the behavior of wild dolphins, more sampling will help truly capture the extent of individual variability described.

Longer deployments will allow a greater period of tag recording time after capture in order to both observe and mitigate any potential after-effects on the data. More overnight tagging will also permit study of interesting behavioral contrasts, such as potential diurnal patterns. Furthermore, conducting focal follows for longer periods of time will help provide more diversity in behavioral context to accompany acoustic events. For example, it would have been particularly useful to have focal observations during the suspected attempts to nurse associated with calf signature whistle concentrations described in Chapter Three, and linking vocal behavior of specific tagged individuals with observations of group encounters may provide us with an unprecedented 
view of how social communication mediates a variety of interactions within fissionfusion societies.

In combination with the longer deployments, a greater sample size will better characterize individual variability and improve statistical power. In particular the model fitted in Chapter Three could benefit from a larger number of separations and reunions that contained signature whistles. Clearer contrasts between the vocal behavior of different demographic groups might also emerge with a larger sample size.

Both of these sampling increases are coming; it will simply take several more years. The principal challenge of an increased sample size is that we are probably already operating at the limit of what it is possible to audit manually; therefore, increased quantities of data will require more automated analysis methods.

In tandem with increased sampling, interactive playback experiments could provide a method to test specific functions of different call types. Playbacks are a powerful tool for exploring acoustic behavior. They have a history of being used in bottlenose dolphins, for example to show that animals respond more strongly to signature whistles of closely-associated animals (Sayigh et al., 1999), often by producing their own signature whistle (Nakahara \& Miyazaki, 2011; King et al., 2014).

Applying the experimental manipulations of playbacks to the cohesion call (Chapter Three) and echolocation monitoring (Chapter Four) hypotheses presented in this thesis would allow them to be further investigated. For example, during a natural separation between a simultaneously-tagged pair of close associates, researchers could position the follow vessel between the animals and play one animal's signature whistle to its partner. If signature whistles function as a reunion call, we would expect the playback 
to increase the probability of a reunion. Alternatively, to test the identity advertisement hypothesis, we could wait until a reunion began and play a signature whistle that did not correspond to the partner. We might expect that this would halt the reunion or lead to further vocal activity seeking confirmation. This reaction could be contrasted with reunions where the expected signature whistle was played. Finally, to investigate the echolocation monitoring hypothesis that an animal's need to reunite is reduced when it can hear its partner's echolocation clicks, we could wait for a separation and play back sonar sounds to one or both partners and observe whether the separations were continued without signature whistling for longer than times with no clicks.

To begin to study cohesion in wild dolphins I needed to distill an extremely complex situation down to focus on the relationships and situations where mechanisms for maintain cohesion were most likely to be expressed. This limited analysis to closelybonded pairs. However, in the future it may be possible to begin moving back towards larger and larger groups. Mother-calf pairs are the simplest possible situation: there are only two animals, and both we as observers and they as animals know they are alone, so if the dolphins are using an echolocation monitoring strategy of the kind described in Chapter Four and hear sonar clicks they did not produce, they can be certain they came from the partner. The situation would be much more complex if there were 4,5,6 or even a hundred animals, and that would likely require an even more prominent role for an individually-distinctive signal such as a signature whistle.

The simultaneous tagging methods described in this thesis work beautifully in a simple dyad, and reducing the complexity of delphinid societies was essential to gain an entry point into the study of cohesion. However, given the challenges for extrapolating 
from individual to population data exemplified by the large variation in vocal behavior described in Chapter Two, it will be important to start building towards working with larger and larger groups.

Some of the more exciting possibilities for future work stem from the combination of all of the methods developed in this thesis together, in order to get at cohesion in more complex groups. Perhaps this could occur with four or five simultaneously-tagged dolphins amidst a larger group of untagged animals. If the geometry of these tagged animals is defined, then they can form an array of hydrophones that can be used to locate calls of untagged animals. Then the stereo camera system could be used to capture geodetic positions of every tagged and untagged animal each time they surface in order to fill in an even more complete behavioral picture of cohesion in a more natural group. 


\subsection{REFERENCES}

Connor, R. C., Mann, J., Tyack, P. L., \& Whitehead, H. (1998). Social evolution in toothed whales. Trends in Ecology \& Evolution, 13(6), 228-232.

Connor, R. C., Wells, R. S., Mann, J., \& Read, A. J. (2000). The Bottlenose Dolphin. In J. Mann, R. C. Connor, P. L. Tyack \& H. Whitehead (Eds.), Cetacean Societies: Field studies of whales and dolphins (pp. 91-126). Chicago: Univ. Chicago Press.

Cook, M. L., Sayigh, L. S., Blum, J. E., \& Wells, R. S. (2004). Signature-whistle production in undisturbed free-ranging bottlenose dolphins (Tursiops truncatus). Proceedings of the Royal Society B: Biological Sciences, 271(1543), 1043-1050.

Da Cunha, R. G. T., \& Byrne, R. W. (2009). The use of vocal communication in keeping the spatial cohesion of groups: Intentionality and specific functions. In P. A. Garber, A. Estrada, J. C. Bicca-Marques, E. W. Heymann \& K. B. Strier (Eds.), South American Primates (pp. 341-363): Springer New York.

Heithaus, M. R. (2001). Shark attacks on bottlenose dolphins (Tursiops aduncus) in Shark Bay, Western Australia: Attack rate, bite scar frequencies and attack seasonality. Marine Mammal Science, 17(3), 526-539.

Janik, V. M. (2009). Acoustic communication in delphinids. In M. Naguib, V. Janik, N. Clayton \& K. Zuberbuhler (Eds.), Advances in the Study of Behavior (Vol. 40, pp. 123-157): Academic Press.

Janik, V. M., \& Sayigh, L. S. (2013). Communication in bottlenose dolphins: 50 years of signature whistle research. Journal of comparative physiology A, 199(6), 479-489.

Janik, V. M., \& Slater, P. J. (1998). Context-specific use suggests that bottlenose dolphin signature whistles are cohesion calls. Animal Behaviour, 56, 829-838.

King, S. L., Harley, H. E., \& Janik, V. M. (2014). The role of signature whistle matching in bottlenose dolphins, Tursiops truncatus. Animal Behaviour, 96, 79-86.

Mann, J., Connor, R. C., Tyack, P. L., \& Whitehead, H. (2000). Cetacean Societies. Field studies of dolphins and whales. Chicago: University of Chicago Press.

Nakahara, F., \& Miyazaki, N. (2011). Vocal exchanges of signature whistles in bottlenose dolphins (Tursiops truncatus). Journal of ethology, 29(2), 309-320.

Sayigh, L. S., Tyack, P. L., Wells, R. S., Solow, A. R., Scott, M. D., \& Irvine, A. B. (1999). Individual recognition in wild bottlenose dolphins: a field test using playback experiments. Animal Behaviour, 57(1), 41-50.

Smolker, R., Mann, J., \& Smuts, B. (1993). Use of signature whistles during separations and reunions by wild bottlenose dolphin mothers and infants. Behavioral Ecology and Sociobiology, 33(6), 393-402.

Wahlberg, M., Jensen, F. H., Soto, N. A., Beedholm, K., Bejder, L., Oliveira, C., . . . Madsen, P. T. (2011). Source parameters of echolocation clicks from wild bottlenose dolphins (Tursiops aduncus and Tursiops truncatus). Journal of the Acoustical Society of America, 130(4), 2263-2274.

Watwood, S. L., Owen, E. C. G., Tyack, P. L., \& Wells, R. S. (2005). Signature whistle use by temporarily restrained and free-swimming bottlenose dolphins, Tursiops truncatus. Animal Behaviour, 69, 1373-1386. 